\title{
New limits on light hidden sectors from fixed-target experiments
}

\author{
David E. Morrissey ${ }^{a}$ and Andrew P. Spray ${ }^{b}$ \\ ${ }^{a}$ TRIUMF, 4004 Wesbrook Mall, \\ Vancouver, BC V6T 2A3, Canada \\ ${ }^{b} A R C$ Centre of Excellence for Particle Physics at the Terascale, \\ School of Physics, University of Melbourne, Victoria 3010, Australia \\ E-mail: dmorri@triumf.ca, andrew.spray@coepp.org.au
}

ABSTRACT: New physics can be light if it is hidden, coupling very weakly to the Standard Model. In this work we investigate the discovery prospects of Abelian hidden sectors in lower-energy fixed-target and high-precision experiments. We focus on a minimal supersymmetric realization consisting of an Abelian vector multiplet, coupled to hypercharge by kinetic mixing, and a pair of chiral Higgs multiplets. This simple theory can give rise to a broad range of experimental signals, including both commonly-studied patterns of hidden vector decay as well as new and distinctive hidden sector cascades. We find limits from the production of hidden states other than the vector itself. In particular, we show that if the hidden Abelian symmetry is higgsed, and the corresponding hidden Higgs boson has visible decays, it severely restricts the ability of the hidden sector to explain the anomalous muon magnetic moment.

KeYwords: Beyond Standard Model, Supersymmetric Standard Model

ARXIV EPRINT: 1402.4817 


\section{Contents}

1 Introduction 2

2 A minimal supersymmetric hidden sector 3

2.1 Hidden masses 3

2.2 Hidden couplings and decays 4

2.3 Benchmark slopes 5

3 Non-fixed target limits $\quad 6$

3.1 Model-independent limits $\quad 6$

$\begin{array}{lll}3.2 & \text { Meson factories } & 7\end{array}$

$\begin{array}{lll}3.3 & \text { Cosmology and astrophysics } & 9\end{array}$

4 Electron fixed target experiments 11

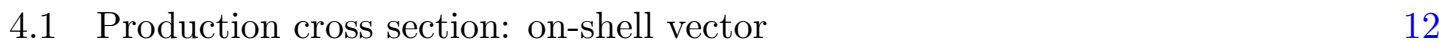

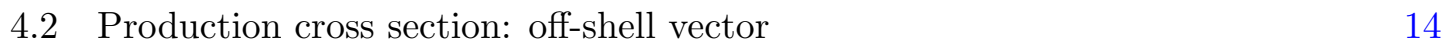

$\begin{array}{lll}4.3 & \text { Expected number of signal events } & 16\end{array}$

$\begin{array}{lll}4.4 & \text { Current and prospective experiments } & 19\end{array}$

$\begin{array}{lll}4.5 & \text { Limits } & 21\end{array}$

5 Hadronic fixed target experiments $\quad 24$

5.1 Production 24

5.2 Acceptances 27

$\begin{array}{lll}5.3 \text { Experiments } & 29\end{array}$

5.4 Limits 31

$6 \quad$ Implications at the LHC $\quad 36$

$\begin{array}{lll}7 & \text { Conclusions } & 38\end{array}$

A Masses and mixings in the hidden sector $\quad 42$

A.1 Masses for $\epsilon \rightarrow 0 \quad 42$

A.2 Mixing with the MSSM 43

A.3 Decays of the light scalar 44

B Details of hidden vector production at electron beam dumps 46

$\begin{array}{ll}\text { B.1 Effective flux } & 47\end{array}$

B.2 Off-shell production 48

B.3 Vector decay widths $\quad 52$

C Hidden sector-visible sector scattering $\quad 52$ 


\section{Introduction}

New physics beyond the Standard Model (SM) could take many forms. To be consistent with existing experiments, any new particles must either be heavier than the electroweak scale or they must interact very weakly with us [1]. Lower-energy experiments with a very high precision or intensity are particularly well-suited to discovering the second possibility when the characteristic mass of the new physics lies below the electroweak scale $[2,3]$. The results of such experiments may also provide guidance for future searches at high energies.

An interesting variety of new physics below the electroweak scale is an exotic Abelian gauge force $\mathrm{U}(1)_{x}$ that couples to the SM exclusively through a small kinetic mixing with hypercharge $[4,5]$

$$
\mathscr{L} \supset-\frac{\epsilon_{Y}}{2} X_{\mu \nu} B^{\mu \nu},
$$

where $X_{\mu \nu}=\left(\partial_{\mu} X_{\nu}-\partial_{\nu} X_{\mu}\right)$ and $X^{\mu}$ is the $\mathrm{U}(1)_{x}$ gauge boson. If the only new particle is the massless $X^{\mu}$, it can be rotated with the photon such that one linear combination decouples from the theory [4]. However, if there are new matter fields charged under $\mathrm{U}(1)_{x}$ they will develop millicharges under this rotation [4]. Since light millicharged matter is very strongly constrained by direct searches and astrophysical observations [6,7], we will focus on a theory where $\mathrm{U}(1)_{x}$ is spontaneously broken.

The simplest way to break $\mathrm{U}(1)_{x}$ is with a hidden Higgs field. In this work we study a minimal supersymmetric theory in which the new gauge force is broken well below the weak scale by a pair of hidden Higgs bosons. With supersymmetry, the relatively low breaking scale can be natural if the hidden sector feels superymmetry breaking less strongly than the visible SM sector and $\epsilon$ is sufficiently small [8-15]. The hidden sector will also contain a dark matter candidate in the presence of $R$-parity. While naturalness and dark matter are attractive features, our primary motivation for investigating this particular model is that it provides a simple but non-minimal theory of a light hidden sector.

Light hidden vectors interacting with the SM through the kinetic mixing interaction of eq. (1.1) have been studied extensively in recent years. The two scenarios that have received the most attention are minimal models where the new massive vector $Z^{x}$ decays primarily to the SM [16-19] or to a pair of dark matter particles [20-24]. These decay channels can also occur in the non-minimal theory we study here, but other decay channels can be dominant as well. In particular, we investigate phases of the theory where the leading vector decays are $Z^{x} \rightarrow h_{1}^{x} A^{x}$, where $h_{1}^{x}$ and $A^{x}$ are hidden Higgs bosons, and $Z^{x} \rightarrow \chi_{1}^{x} \chi_{2}^{x}$, where $\chi_{1}^{x}$ and $\chi_{2}^{x}$ are hidden neutralinos. The experimental signals of these additional channels have not received as much attention as the purely visible or invisible channels (although see ref. [25]). We find that the $h_{1}^{x}, A^{x}$, and $\chi_{2}^{x}$ decay products can be metastable, and that fixed-target experiments are particularly well-suited to finding them.

In this paper, we study the sensitivity of low-energy and high-precision experiments to a minimal supersymmetric Abelian hidden sector, with a focus on fixed-target experiments. We begin in section 2 by defining the theory and characterizing the mass eigenstates and their decay modes. We also formulate four distinct sets of benchmark parameters to be studied in the sections to come. In section 3 , we investigate the existing limits on the 
theory from non-fixed-target experiments, including precision tests, meson factories, and cosmology. Next, in sections 4 and 5 we study the sensitivities of current and future electron and hadron fixed target experiments to the theory in its various phases. The implications of our results for the LHC are discussed in section 6 , and we conclude in section 7 . Some technical details about the theory and our calculation of vector production and detection in fixed target experiments are collected in the appendices A, B and C.

\section{A minimal supersymmetric hidden sector}

We consider the minimal supersymmetric hidden sector formulated in refs. [12, 26] consisting of a $\mathrm{U}(1)_{x}$ vector multiplet $X$ together with a pair of chiral multiplets $H$ and $H^{\prime}$ with charges $x_{H, H^{\prime}}= \pm 1$ that develop vacuum expectation values (VEVs). In addition to a minimal Kähler potential for $H$ and $H^{\prime}$, the interactions in the hidden sector are taken to be:

$$
\mathscr{L}_{\text {hid }}=\mathscr{L}_{V}+\mathscr{L}_{W}+\mathscr{L}_{\text {soft }},
$$

with the vector terms

$$
\mathscr{L}_{V}=\int d^{2} \theta\left(\frac{1}{4} B^{\alpha} B_{\alpha}+\frac{1}{4} X^{\alpha} X_{\alpha}+\frac{1}{2} \frac{\epsilon}{\cos \theta_{W}} B^{\alpha} X_{\alpha}\right)+\text { h.c. },
$$

the superpotential terms

$$
\mathscr{L}_{W}=\int d^{2} \theta \mu^{\prime} H H^{\prime}
$$

and the soft terms

$$
-\mathscr{L}_{\text {soft }}=m_{H}^{2}|H|^{2}+m_{H^{\prime}}^{2}\left|H^{\prime}\right|^{2}-\left(b^{\prime} H H^{\prime}+\text { h.c. }\right)-M_{x} \tilde{X}^{2} .
$$

Note that the kinetic mixing has been written in terms of $\epsilon \equiv \epsilon_{Y} \cos \theta_{W}$, where $\theta_{W}$ is the Weinberg angle, to agree with the most common convention. This is a different normalisation to that made in ref. [26] (which took $\epsilon \equiv \epsilon_{Y}$ ). We assume $\epsilon \ll 1$ and take the dimensionful $\mu^{\prime}$ and soft terms to be at or below a GeV. This choice is consistent with supersymmetric naturalness for sufficiently small $\epsilon[12,26]$, and predicts a hidden vector mass of the same order. The visible sector is assumed to be the minimal supersymmetric standard model (MSSM).

\subsection{Hidden masses}

For appropriate values of the model parameters, the scalar components of $H$ and $H^{\prime}$ develop VEVs,

$$
\langle H\rangle=\eta \sin \zeta, \quad\left\langle H^{\prime}\right\rangle=\eta \cos \zeta,
$$

with $\zeta \in[0, \pi / 2]$. As a result, the hidden vector $X_{\mu}$ obtains a mass equal to

$$
m_{x}=\sqrt{2} g_{x} \eta
$$

We will denote this state $Z^{x}$. 
Hidden symmetry breaking also gives rise to mass mixing among the scalar and fermion sectors of the theory. The remaining bosonic mass eigenstates are a pair of real scalars $h_{1}^{x}$ and $h_{2}^{x}$ with masses $m_{h_{1}^{x}} \leq m_{h_{2}^{x}}$, and a pseudoscalar $A^{x}$ of mass $m_{A^{x}} .{ }^{1}$ The fermionic mass eigenstates are mixtures of the $\mathrm{U}(1)_{x}$ gaugino and the hidden higgsinos: $\chi_{1}^{x}, \chi_{2}^{x}, \chi_{3}^{x}$ with masses $\left|m_{\chi_{1}^{x}}\right|<\left|m_{\chi_{2}^{x}}\right|<\left|m_{\chi_{3}^{x}}\right|$. A more detailed exposition of hidden-sector mass mixing can be found in ref. [26] and appendix A.

When symmetry breaking occurs in the hidden sector, a convenient basis of parameters for the theory is $\left\{g_{x}, \mu^{\prime}, m_{x}, m_{A^{x}}, M_{x}, \tan \zeta, \epsilon\right\}$. Note as well that the structure of the hidden Higgs sector mirrors that of the neutral Higgs of the MSSM [27]. As such, we obtain $m_{h_{1}^{x}}<m_{x}, m_{A^{x}}<m_{h_{2}^{x}}$ at tree-level, and we expect this ordering to persist at loop level provided $g_{x}$ is small and $\mu^{\prime}, M_{x} \sim m_{x}$. We will also assume that $R$-parity is conserved and that the gravitino is moderately heavy, so that $\chi_{1}^{x}$ is a stable lightest superpartner (LSP).

\section{$2.2 \quad$ Hidden couplings and decays}

Particles in the hidden sector interact with each other through the gauge coupling $g_{x}$ and with the visible (MSSM) sector via the kinetic mixing parameter $\epsilon$. For $e \epsilon \ll g_{x}$, as we assume here, hidden states nearly always decay to other hidden states when kinematically allowed. However, when the hidden channels have been exhausted, decays to SM can dominate giving rise to new signals in low- and high-energy experiments.

The main connector to the SM is the hidden vector $Z^{x}$, and it can decay in various ways depending on the mass spectrum of the hidden sector. The experimental signatures of the theory can be classified according to the most probable decay mode of the vector. We will consider the four most important possibilities:

$$
Z^{x} \rightarrow\left\{\begin{array}{l}
\mathrm{SM}+\mathrm{SM} \\
\chi_{1}^{x}+\chi_{1}^{x}, \\
h_{1}^{x}+A^{x}, \\
\chi_{i}^{x}+\chi_{j}^{x}, i>1
\end{array}\right.
$$

Below, we will define four benchmark parameter slopes that realize each of these four cases.

An interesting feature of this theory is that the lightest scalar $h_{1}^{x}$ is always lighter than the vector and all the other scalars (at weak coupling). The only possible hidden channels are

$$
h_{1}^{x} \rightarrow \chi_{i}^{x} \chi_{j}^{x}
$$

When all these channels are forbidden, $m_{h_{1}^{x}}<2 m_{\chi_{1}^{x}}$, the $h_{1}^{x}$ state will decay to the SM. The two main contributions to the decay come from mass mixing with the MSSM Higgs bosons and from a loop of hidden vectors connecting to a pair of SM fermions. The effective Higgs mixing angle goes like $\epsilon\left(m_{x} / m_{h^{0}}\right)$, where $m_{h^{0}}$ is the mass of the lighter MSSM Higgs boson, while the vector loop is proportional to $\epsilon^{2}$. In computing the $h_{1}^{x}$ decay width, we find that the Higgs mixing contribution tends to dominate over the loop contribution above the dimuon threshold. However, both contributions have significant suppressions, and the

\footnotetext{
${ }^{1}$ We neglect $\mathrm{CP}$ violation in the present work.
} 
$h_{1}^{x}$ state is nearly always long-lived on collider time scales. More details can be found in appendix A.

Among the heavier scalars, there are a number of possible decay channels:

$$
\begin{aligned}
& A^{x} \rightarrow h_{1}^{x} Z^{x(*)}, \chi_{i}^{x} \chi_{j}^{x}, \\
& h_{2}^{x} \rightarrow A^{x} Z^{x(*)}, Z^{x} Z^{x(*)}, h_{1}^{x} h_{1}^{x(*)}, \chi_{i}^{x} \chi_{j}^{x},
\end{aligned}
$$

where $(*)$ denotes that the decay product is potentially off-shell. These decays tend to be prompt when they are two-body, but can become slow when they are forced to be threebody with a SM final state (e.g. $A^{x} \rightarrow h_{1}^{x} Z^{x *} \rightarrow h_{1}^{x}(f \bar{f})$ where $f$ is a charged SM fermion).

With our assumption of $R$-parity conservation, the lightest hidden neutralino is the stable LSP (in the absence of a light gravitino). The heavier hidden neutralinos decay according to

$$
\chi_{i}^{x} \rightarrow \chi_{j}^{x}+\left\{Z^{x(*)}, A^{x(*)}, h_{1,2}^{x(*)}\right\} .
$$

Again, the branching fractions of these decays depend on the spectrum and mixing of hidden states. Note as well that the lightest MSSM superpartner can decay to the hidden sector through gauge kinetic mixing (e.g. $\chi_{1}^{0} \rightarrow \chi_{1}^{x}+\left\{Z^{x}, h_{1}^{x}, h_{2}^{x}, A^{x}\right\}$ ).

\subsection{Benchmark slopes}

This minimal supersymmetric hidden sector can give rise to a broad range of experimental signatures. To illustrate this range, we investigate the signals of four sample slopes corresponding to four different primary decay modes of the hidden vector $Z^{x}$. For each slope, we set $\tan \zeta=3$ and $\alpha_{x}=\alpha$, and we take the remaining model parameters to be fixed ratios of the vector mass $m_{x}$. These ratios and the resulting mass spectra (in units of $m_{x}$ ) are listed in table 1.

Each of the four slopes corresponds to a different dominant decay channel for the hidden vector boson. They are:

\section{A. $\mathbf{Z}^{\mathrm{x}} \rightarrow \mathbf{S M}+\mathbf{S M}$}

The vector has no two-body decays within the hidden sector, and as a result decays almost entirely through gauge kinetic mixing with the photon. This scenario occurs readily for $m_{x}<M_{x}, \mu^{\prime}, m_{A^{x}}$, and has been studied in great detail [1]. Limits on this scenario are dominated by direct production of the vector followed by its decay to visible SM final states. We find some additional limits at fixed-target experiments based on the production of the lightest Higgs $h_{1}^{x}$ through an off-shell vector, followed by observing either the decay or the scattering of the scalar. Decays of the lighter scalar $h_{1}^{x}$ are addressed above and in appendix A.

B. $\mathbf{Z}^{\mathbf{x}} \rightarrow \chi_{1}^{\mathbf{x}} \chi_{1}^{\mathbf{x}}$

This channel is now the only two-body hidden mode, and it dominates over the $S M+S M$ channel (for $e \epsilon \ll g_{x}$ ). It is likely to occur for $M_{x}<m_{x}<m_{A^{x}}, \mu^{\prime}$. The final state is invisible, and has been searched for in two ways: missing energy signatures in meson factories; and elastic scattering of $\chi_{1}^{x}$ particles in the detector of 


\begin{tabular}{|ccccccccc|}
\hline Case & $M_{x}$ & $\mu^{\prime}$ & $m_{A^{x}}$ & $m_{h_{1}^{x}}$ & $m_{h_{2}^{x}}$ & $m_{\chi_{1}^{x}}$ & $m_{\chi_{2}^{x}}$ & $m_{\chi_{3}^{x}}$ \\
\hline A & 3.0 & 4.0 & 2.0 & 0.76 & 2.10 & 2.50 & -4.02 & 4.53 \\
B & 1.0 & 1.5 & 1.5 & 0.73 & 1.65 & 0.39 & -1.59 & 2.20 \\
C & 1.5 & 2.0 & 0.5 & 0.38 & 1.05 & 0.86 & -2.06 & 2.69 \\
D & 3.0 & 0.5 & 1.5 & 0.73 & 1.65 & 0.24 & 0.57 & 3.33 \\
\hline
\end{tabular}

Table 1. Sample parameters and particle masses for the four benchmark slopes. All quantities are listed in units of the vector mass $m_{x}$, and we also fix $\alpha_{x}=\alpha$ and $\tan \zeta=3$.

fixed-target experiments [1]. As for the previous benchmark, we find some new limits based on the decay of the lightest Higgs $h_{1}^{x}$, produced through an off-shell vector.

\section{C. $\mathbf{Z}^{\mathrm{x}} \rightarrow \mathrm{h}_{1}^{\mathrm{x}} \mathrm{A}^{\mathrm{x}}$}

The initial vector decay is prompt, but the $h_{1}^{x}$ and $A^{x}$ products decay further to the SM and are typically delayed. The pseudoscalar decays via $A^{x} \rightarrow h_{1}^{x}+Z^{x *}$ with $Z^{x *} \rightarrow S M+S M$, and it can be slow as well. An ordering $m_{A^{x}}<m_{x}<\mu^{\prime}, M_{x}$ is likely to produce this decay channel. Depending on their decay lengths, the production of long-lived $A^{x}$ and $h_{1}^{x}$ states via the vector can lead to missing energy signatures in meson factories. It can also lead to either highly displaced visible decays or elastic scattering signals in the detectors of fixed-target experiments. This case has only been studied in ref. [25] within a simplified model of the hidden sector.

D. $\mathbf{Z}^{\mathrm{x}} \rightarrow \chi_{\mathbf{1}}^{\mathrm{x}}+\chi_{\mathbf{2}}^{\mathrm{x}}$

In this case, the vector has a pair of two-body hidden decay channels to neutralinos with branching fractions $B R\left(\chi_{1}^{x} \chi_{2}^{x}\right) \simeq 0.94$ and $B R\left(\chi_{1}^{x} \chi_{1}^{x}\right) \simeq 0.06$. The presence of two lighter hidden neutralinos occurs readily when $\mu^{\prime}<m_{x}<M_{x}, m_{A^{x}}$. The heavier of the two states tends to have a larger gaugino fraction, and this favours the $\chi_{1}^{x} \chi_{2}^{x}$ channel over the $\chi_{1}^{x} \chi_{1}^{x}$. The $\chi_{2}^{x}$ produced in the vector decay goes to $\chi_{2}^{x} \rightarrow \chi_{1}^{x}+Z^{x^{*}}$ with $Z^{x^{*}} \rightarrow S M+S M$, and can be long-lived. This can lead to visible decays in fixedtarget experiments. We also have elastic scattering signals at these experiments from $\chi_{1}^{x}$ and (for long enough lifetimes) from $\chi_{2}^{x}$. We do not know of any previous studies of this decay channel.

In the sections to follow, we will investigate the current experimental bounds on these four scenarios.

\section{Non-fixed target limits}

To begin, we collect limits on the four scenarios from precision tests, meson factories, and cosmology.

\subsection{Model-independent limits}

Precision electroweak observables and lepton magnetic moments provide important limits on the mass and kinetic mixing of the hidden vector that are independent of how it decays. We review here the model-independent constraints that apply to all four cases. 
Kinetic mixing ties the hidden vector to the hypercharge vector, and this induces a mixing between $Z^{x}$ and the $Z^{0}$ after electroweak and hidden symmetry breaking [28, 29]. A global fit to precision data in the presence of this mixing was performed in ref. [30], and the limit for $m_{x} \lesssim 10 \mathrm{GeV}$ was found to be

$$
\epsilon<(0.03) c_{W} \simeq 0.026
$$

The leading effects of a kinetically-mixed hidden vector on the anomalous magnetic moments $a_{\ell}=(g-2)_{\ell}$ of the electron and muon were computed in ref. [16]. The current experimental status for the muon is [31]

$$
\Delta a_{\mu}=a_{\mu}^{o b s}-a_{\mu}^{S M}=(287 \pm 80) \times 10^{-11} .
$$

We demand that $0<\Delta a_{\mu}<447 \times 10^{-11}$, so as to lie within $2 \sigma$ of the observed value while also allowing for consistency with the SM. The additional contribution due to the hidden vector tends to increase the predicted value of $\Delta a_{\mu}$, and an interesting possibility is that a hidden vector is the source of the apparent (3.6 $\sigma)$ discrepancy with the SM [16].

For the anomalous magnetic moment of the electron, we use [32-34]

$$
\Delta a_{e}=(-10.5 \pm 8.1) \times 10^{-13},
$$

and we impose the $3 \sigma$ limit $-34.8<\Delta a_{e} \cdot 10^{13}<13.8$. This range uses the updated direct measurement of $a_{e}$ [35] compared with the SM prediction [36] computed with the value of of $\alpha$ derived from atomic measurements in rubidium [37]. The combined precision electroweak and magnetic moment bounds are shown in figures 1 and 2 .

\subsection{Meson factories}

New light states have been searched for in a diverse range of meson factories, including BaBar, Belle, KLOE, and in rare Kaon decays at Brookhaven. We examine the bounds they imply for the theory.

Case A. The direct signals of the vector in this case have been studied previously. For $2 m_{\mu}<m_{x}<10.335 \mathrm{GeV}$, there are strong limits from the BaBar search for an exotic pseudoscalar $a^{0}$ via $\Upsilon(3 s, 2 s) \rightarrow \gamma a^{0}$ with $a^{0} \rightarrow \mu^{+} \mu^{-}$[38, 39] when applied to the continuum process $e^{+} e^{-} \rightarrow \gamma Z^{x}$ with $Z^{x} \rightarrow \mu^{+} \mu^{-}[19,40]$. For $m_{x} \in[100,400] \mathrm{MeV}$, the strongest bounds come from the KLOE search for $\phi \rightarrow \eta Z^{x}$ with $Z^{x} \rightarrow e^{+} e^{-}[41,42]$. When $m_{x}<100 \mathrm{MeV}$, WASA-at-COSY is most constraining based on $\pi^{0} \rightarrow Z^{x} \gamma$ with prompt $Z^{x} \rightarrow e^{+} e^{-}$[43]. In all cases, the bound is approximately $\epsilon \lesssim 3 \times 10^{-3}$.

Our supersymmetric realization of this scenario will also induce new decay channels for mesons through the mixing of $h_{1}^{x}$ and $h_{2}^{x}$ with the MSSM Higgs bosons. Limits on this mixing were studied in refs. $[20,44,45]$, where the strongest bounds were found to come from $K^{+} \rightarrow \pi^{+} h_{1}^{x}$ and $B^{+} \rightarrow K^{+} h_{1}^{x}$ with $h_{1}^{x} \rightarrow \mu^{+} \mu^{-}$, invisible. The effective Higgs mixing angle in this theory is proportional to $\epsilon\left(m_{x} / m_{h^{0}}\right)$, where $m_{h^{0}}$ is the SM-like Higgs boson mass (see appendix A). We find that the model-independent and direct limits on $\epsilon$ and $m_{x}$ from the effects of the vector are stronger than those from decays through Higgs mixing for $m_{x}<10 \mathrm{GeV}$. In the same way, no useful limits are found from leptonic heavy meson decays [46]. 

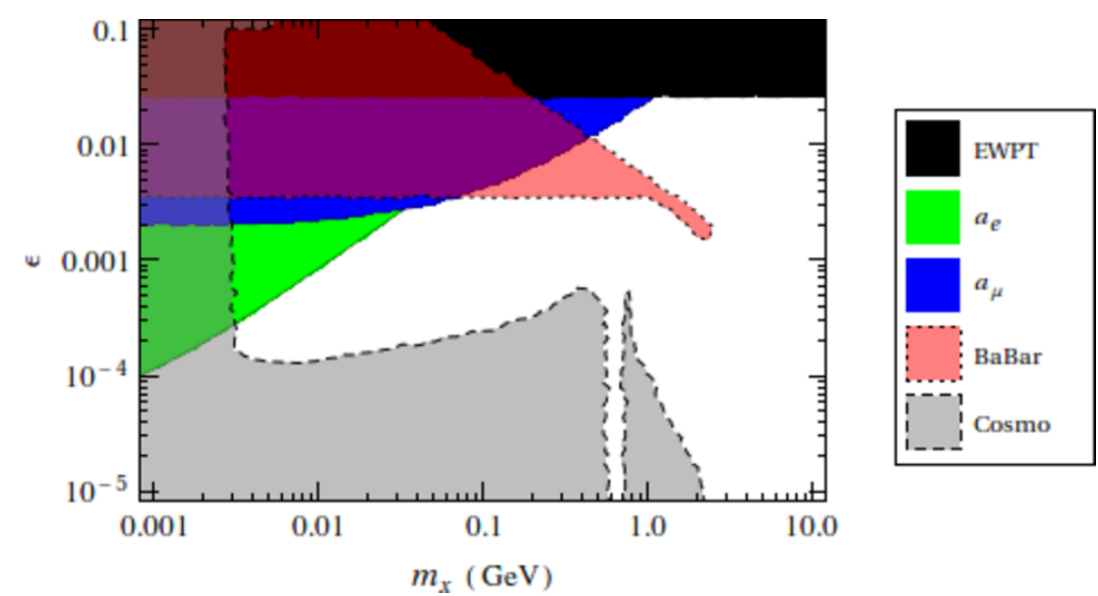

Figure 1. Non-fixed target limits on Case C. The black, green and blue regions show the modelindependent exclusions of section 3.1; the red regions the BaBar constraints discussed in section 3.2; and the grey regions the cosmological limits from section 3.3. The cosmological limits are less robust, as discussed in section 3.3.

Case B. Direct bounds on an invisibly decaying hidden vector were investigated in refs. [22, 24]. The BaBar search for an invisible pseudoscalar in $\Upsilon(3 s) \rightarrow \gamma a^{0}$ with $a^{0} \rightarrow$ invisible [47] can be reinterpreted as a limit on $e^{+} e^{-} \rightarrow \gamma Z^{x}$ with $Z^{x} \rightarrow$ invisible [40], and imply $\epsilon \lesssim(1-4) \times 10^{-3}$ depending on the masses $m_{x}(<8 \mathrm{GeV})$ and $m_{\chi_{1}^{x}}$. The Brookhaven E787 and E949 searches for $K^{+} \rightarrow \pi^{+} Z^{x}$ with $Z^{x} \rightarrow$ invisible $[48,49]$ also limit $\epsilon \lesssim 1 \times 10^{-3}$ in the regions $m_{x} \in[0,120] \cup[160,240] \mathrm{MeV}[22,24]$. No additional bounds are obtained from rare meson decays through Higgs mixing.

Case C. The vector decay $Z^{x} \rightarrow h_{1}^{x} A^{x}$ has not been studied in as much detail as the other cases [25]. Both products can have long lifetimes, and the limits found for Case B can often be applied to this case as well. The BaBar $\Upsilon(3 s) \rightarrow \gamma+$ invisible search imposed a veto on additional activity in the electromagnetic calorimeter and on hits in the muon chamber opposite to the photon direction [47]. To approximate the exclusion implied by this search, we apply the bounds found in refs. [22, 24] with the additional condition that $\overline{\gamma v}_{T} \tau>100 \mathrm{~cm}$ for both $h_{1}^{x}$ and $A^{x}$, where $\overline{\gamma v}_{T}$ is the mean boosted transverse velocity in the lab frame. The value of $100 \mathrm{~cm}$ is the approximate transverse radius of the BaBar electromagnetic calorimeter and muon chamber [50]. We estimate $\overline{\gamma v}_{T}=\bar{p}_{T} / m$ using a simple Monte Carlo simulation that takes into account the angular distribution of the production cross section [51] and the angular acceptance of photons in the search [47]. The exclusions derived in this way are shown in figure 1 together with the model-independent bounds. We also find that the exclusions from $K^{+} \rightarrow \pi^{+}+i n v$ apply here for $\epsilon \lesssim 10^{-2}$, although we do not show them in the figures.

In contrast to searches for invisible decay modes, the existing meson-factory searches for the visible decays of a hidden vector presented in refs. [38, 39, 41-43, 52, 53] do not appear to give any relevant limits on this scenario. These searches tend to focus on leptonic decays, they typically look for one or more resonances, and they often demand that the 


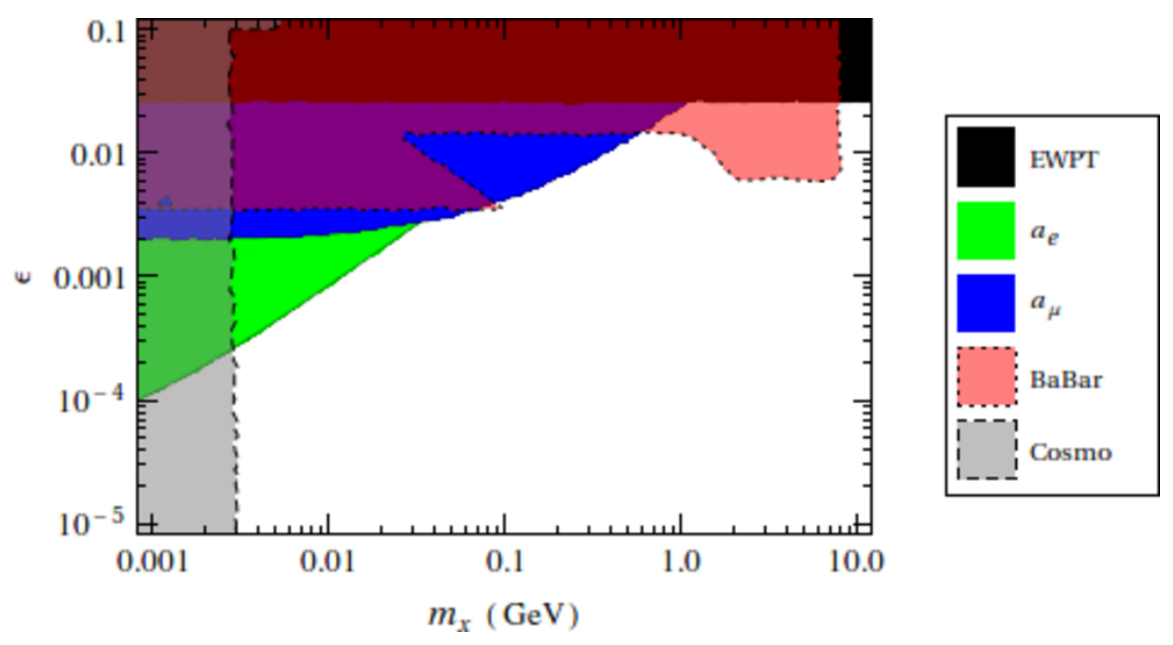

Figure 2. Non-fixed target limits on Case D, in the same format as figure 1.

energies of the visible products reconstruct the mass of the decaying meson or the beam energy. Vector decays in case $\mathrm{C}$ will typically have two visible products and missing energy (for $h_{1}^{x}$ long-lived), or six visible products (for $h_{1}^{x}$ short-lived). When there is missing energy, the energies of the visible products will not reconstruct the total beam or decaying meson energy, as required in refs. [38, 39, 41, 42, 52]. Missing energy will be absent when $h_{1}^{x}$ decays promptly. However, this only occurs when $m_{h_{1}^{x}} \gtrsim \mathrm{GeV}$ where the hidden Higgs has only a very small branching fraction to leptons. This implies that the search of ref. [43] using pion decays, as well as the inclusive leptonic searches of ref. [53], are insensitive. Finally, note that the pseudoscalar $A^{x}$ decay involves an off-shell vector, and its decay products will not reconstruct a sharp resonance.

Case D. The vector now decays primarily to $Z^{x} \rightarrow \chi_{1}^{x} \chi_{2}^{x}$ with $B R_{12} \simeq 0.94$, but also to $Z^{x} \rightarrow \chi_{1}^{x} \chi_{1}^{x}$ with $B R_{11} \simeq 0.06$. In the former channel, the subsequent decay of $\chi_{2}^{x}$ to $\chi_{1}^{x}$ and visible matter via an off-shell $Z^{x}$ can be delayed. As in Case $\mathrm{C}$, we find that meson factory searches for visible decays do not give any useful bounds. However, the BaBar $\Upsilon(3 s) \rightarrow$ $\gamma+$ invisible search of ref. [47] is applicable here. When the $\chi_{2}^{x}$ decay occurs relatively promptly, $\overline{\gamma v_{T}} \tau<100 \mathrm{~cm}$ (with the mean value of $\overline{\gamma v_{T}}=\bar{p}_{T} / m_{\chi_{2}^{x}}$ estimated with a simple Monte Carlo simulation), only the $Z^{x} \rightarrow \chi_{1}^{x} \chi_{1}^{x}$ channel contributes significantly. In this situation we rescale the limits on $\epsilon$ obtained refs. [22, 24] by $1 / \sqrt{B R_{11}}$. For $\overline{\gamma v_{T}} \tau>100 \mathrm{~cm}$, no such rescaling is needed. The exclusions derived in this way are shown in figure 2.

\subsection{Cosmology and astrophysics}

This theory can also be constrained by cosmological observations given a specific assumption for the evolution of the Universe. An obvious requirement for all cases is that the relic density of the lightest hidden neutralino $\chi_{1}^{x}$ not be too large. The relic density calculations of refs. $[54,55]$ are applicable here. In general, the thermal relic density tends to be well below critical for $m_{\chi_{1}^{x}}>m_{x}$ unless $\alpha_{x}$ is also very small, but can become unacceptably large when $m_{\chi_{1}^{x}}<m_{x}$. 
If $\chi_{1}^{x}$ does make up the dark matter, it is typically consistent with existing limits from direct detection. Scattering can occur through the exchange of a hidden vector (via kinetic mixing) or a hidden Higgs boson (via Higgs portal mixing). Since the lightest hidden neutralino is Majorana, vector-mediated scattering corresponds to the effective operator $\left(\bar{\chi}_{1}^{x} \gamma^{\mu} \gamma^{5} \chi\right)\left(\bar{f} \gamma_{\mu} f\right)$, which leads to spin-independent (SI) scattering suppressed by two powers of the dark matter velocity [56], in addition to a factor of $\epsilon^{2}$. The exchange of a hidden Higgs boson will also contribute to SI scattering, now with a suppression factor on the order of $\epsilon^{2}\left(m_{x} m_{Z} / m_{h_{1}^{x}}^{2}\right)^{2}$ relative to SI scattering mediated by the SM Higgs boson. These suppressions lead to direct-detection cross sections well below existing limits from nuclear- [57] and electron-recoil [58, 59] analyses once the precision bounds on $\epsilon$ are applied.

Additional bounds from cosmology can arise if some of the states in the theory are long-lived. Lifetimes greater than $\tau \simeq 0.05 \mathrm{~s}$ will lead to decays after the onset of big-bang nucleosynthesis (BBN) and can potentially alter the abundances of light elements [60,61]. Late decays can also lead to spectral distortions in the cosmic microwave background radiation [62] or an excess in gamma rays [63].

In cases $\mathrm{A}-\mathrm{C}$, the longest-lived state is nearly always the $h_{1}^{x}$ scalar. It decays mainly through mixing with the MSSM Higgs bosons to pairs of SM particles. When $m_{h_{1}^{x}}>2 m_{\pi}$, a significant fraction of these decays will be hadronic and will ultimately create light pions. When $\tau>0.05 \mathrm{~s}$, these pions can scatter with protons to create more neutrons than would otherwise be present $[60,61,64]$. Below the pion threshold, all decays will be purely electromagnetic, producing muons, electrons, or photons. These particles thermalize efficiently with the plasma, and for $\tau \lesssim 10^{4} \mathrm{~s}$, they do so before potentially destroying light nuclei $[60,61,64]$. Thus, longer lifetimes are safe below the pion threshold. In figure 1 we show the regions where $h_{1}^{x}$ decay could be dangerous for nucleosynthesis (or the CMB) based on these considerations. These regions are not necessarily ruled out if the yield $Y=n / s$ of $h_{1}^{x}$ is sufficiently small at the time of decay. However, in a thermal scenario where $h_{1}^{x}$ begins with an equilibrium density and freezes out, the freeze-out process relies on the three-body annihilation mode $h_{1}^{x} h_{1}^{x} \rightarrow Z^{x} Z^{x *}$ and tends to produce a relatively large yield.

The long-lived state in case $\mathrm{D}$ is the next-to-lightest neutralino $\chi_{2}^{x}$, which decays through an off-shell vector. For $\Delta m_{\chi}=m_{\chi_{2}^{x}}-m_{\chi_{1}^{x}}>2 m_{\pi}$, a significant fraction of these decays will be hadronic. For smaller mass differences the decays will be electromagnetic. In figure 2 we show the regions where this decay could potentially affect nucleosynthesis. Note, however, that the transfer reaction $\chi_{2}^{x} \chi_{1}^{x} \rightarrow \chi_{1}^{x} \chi_{1}^{x}$ is generally efficient, and the thermal yield of $\chi_{2}^{x}$ is typically very small.

Further constraints on all four cases can be derived from the observed cooling rate of supernova (SN) 1987A [19, 65, 66]. The hidden vector can be created by thermal scattering within a $\mathrm{SN}$ and generate new energy loss mechanisms. In case A, this vector can be longlived and escape the SN before it decays to the SM, and small values of $\epsilon \lesssim 10^{-7}$ are excluded for $m_{x} \lesssim 100 \mathrm{MeV}$ [19]. In cases B-D, the vector will decay promptly to longlived hidden states such as $h_{1}^{x}$ or $\chi_{1}^{x}$ which can carry energy away if they leave the SN before decaying or scattering. For these cases, we expect limits similar to those found in ref. [66], which studied the bounds on hidden dark matter in the form of a Dirac fermion 


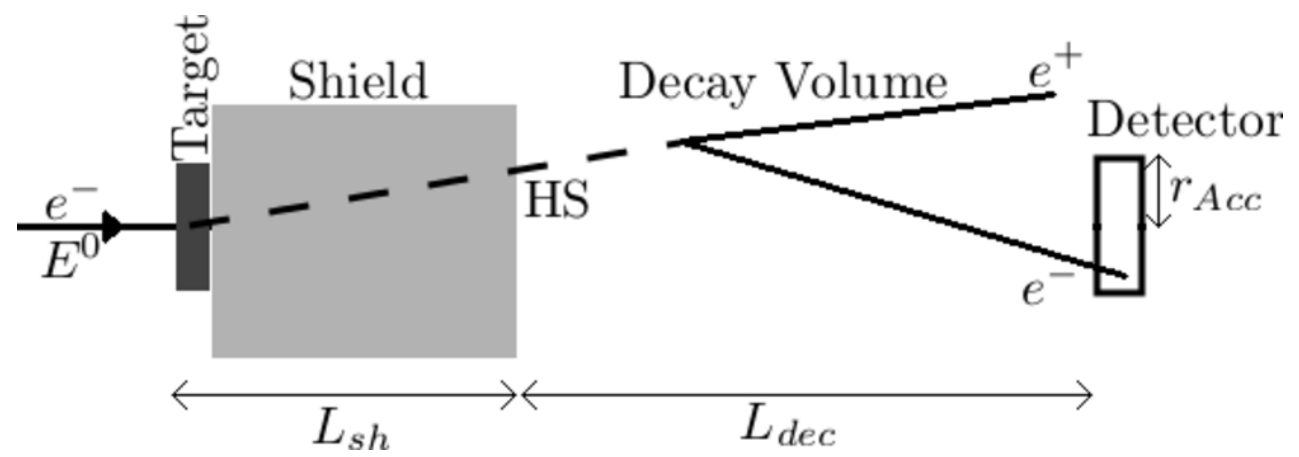

Figure 3. Sketch of the experimental configuration for fixed target searches for visible decays. The electron beam strikes the target, producing a metastable hidden sector state (dashed line); this decays to visible sector particles within the decay volume after passing through the shield. A signal requires that at least one of the visible decay products strike the detector.

or a complex scalar charged under $\mathrm{U}(1)_{x}$. A potential important difference in the present case is that the metastable states interact differently with the vector boson, and may be less likely to scatter before exiting the SN. We defer an analysis of this effect to a future work.

\section{Electron fixed target experiments}

Fixed target experiments using electron beams have played an important role in studies of hidden vectors. Significant limits have been set when the gauge bosons decay directly to the SM [19, 67-70]. Additionally, a number of experiments have been proposed to extend the search reach based on both visible [71-74] and invisible [22] decays. It is therefore natural to examine how they constrain the more general hidden sectors we consider here.

Most searches require hidden states decaying to the SM, so we begin by considering that case. The experimental geometry is sketched in figure 3. A signal requires the production of a metastable state at the interaction point, which decays to visible sector particles (typically required to be leptons) within the decay volume. In previous work, the longlived state was the hidden vector itself; the lifetime requirement leads to limits being set at small kinetic mixing and/or low mass.

However, as already discussed in section 2, our model displays a broader range of phenomenology. Of our four benchmarks, only in Case A do the standard limits apply. In all other Cases, the vector $Z^{x}$ decays promptly to the hidden sector. Instead, limits may be derived from other metastable particles: the lightest scalar $h_{1}^{x}$ (Cases A-C), pseudoscalar $A^{x}$ (Case C) and next-to-lightest fermion $\chi_{2}^{x}$ (Case D). The subsequent limits at electron beam dumps have not been previously studied.

Another class of searches look for the production of invisible states at fixed target experiments. These have been proposed as a way to search for light dark matter [22]. The experimental geometry is sketched in figure 4. Production of hidden sector states proceeds as before, producing either stable or sufficiently long-lived hidden particles. The experiment would seek to observe the quasi-elastic scattering of these states with nuclei within the detector, similar to searches for the direct detection of dark matter. Such a 


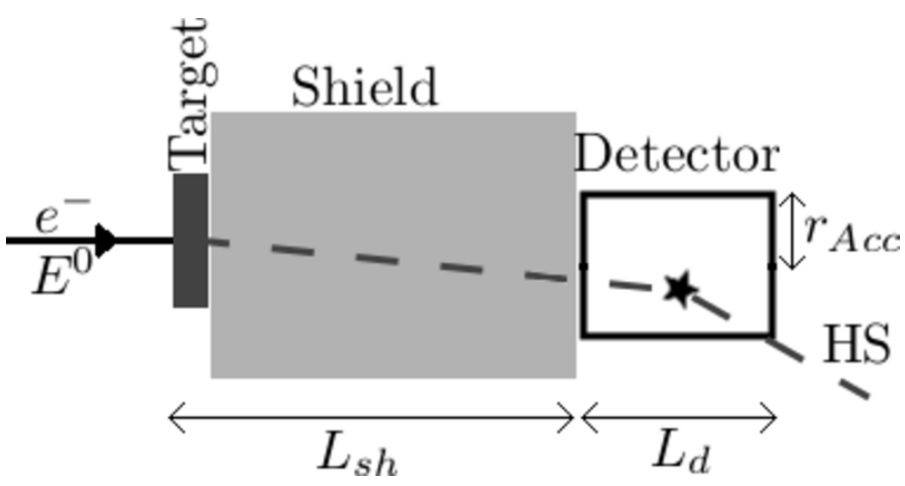

Figure 4. Sketch of the experimental configuration for fixed target searches for invisible final states. The electron beam strikes the target, producing a stable or long-lived hidden sector state (dashed line). This then scatters inside the detector at the starred point.

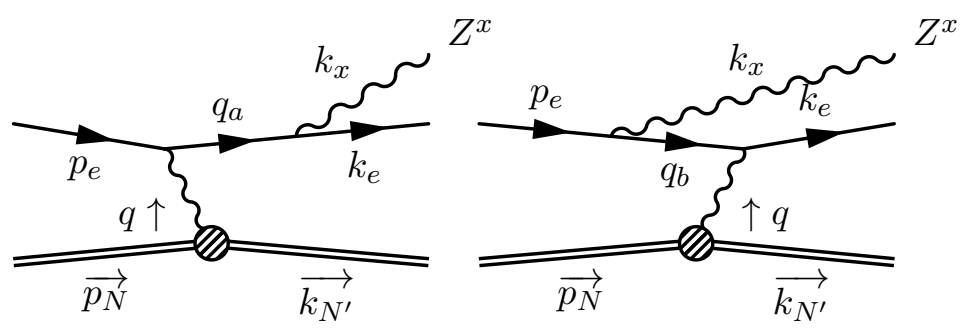

Figure 5. The two leading Feynman diagrams for production of on-shell massive vector in electronfixed target scattering. The double lines represent the initial and final atomic/nuclear states $N$ and $N^{\prime}$.

search is highly relevant in Cases $\mathrm{B}$ and $\mathrm{D}$, when the $Z^{x}$ can decay to the stable lightest fermion $\chi_{1}^{x}$.

After some general comments, we discuss the production of the hidden sector through an on-shell (off-shell) $Z^{x}$ in section 4.1 (4.2). We relate the cross sections to the expected signals in section 4.3, and discuss relevant experiments in section 4.4. The theoretical framework for the production of a hidden vector at electron fixed target experiments is well established [19, 67, 75, 76] (see also ref. [22]), and we outline the main points here to be self-contained and extend the formalism in some points for the analysis at hand. Additional technical details are presented in appendix B. The reader who is interested primarily in results should therefore skip to section 4.5, where we present the combined limits from electron beam dumps for our four benchmark scenarios.

\subsection{Production cross section: on-shell vector}

We first consider the production of an on-shell hidden vector. This has been the usual focus of attention, with a long-lived vector decaying to leptons; that is relevant in Case A. Our interest is on a vector that decays promptly to hidden sector scalars (Case C) or fermions (Cases B and D).

There are two Feynman diagrams at leading order, shown in figure 5. The improved Weizsacker-Williams (WW) approximation [75] lets us factor out the dependence on the structure of the target into an integral over form factors. For an electron of energy $E_{e}$, this 


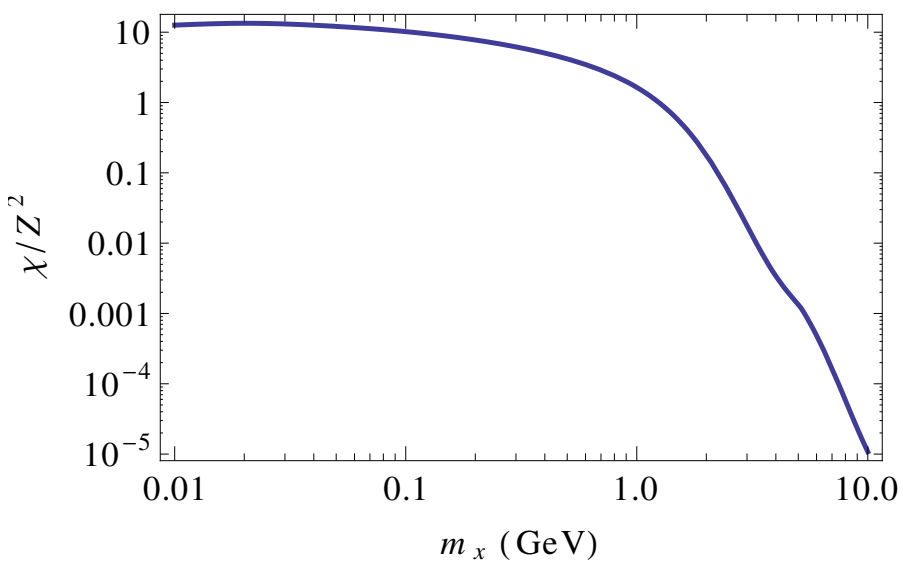

Figure 6. The effective flux $\chi$ for the E137 experiment, normalised to the target atomic number $Z=13$.

approximation is valid when [67]

$$
m_{e} \ll m_{x} \ll E_{e} \quad \text { and } \quad E_{x} \theta_{x}^{2} \ll E_{e},
$$

where $E_{x}, \theta_{x}$ are respectively the energy and scattering angle of the hidden vector. The production differential cross section for the full process is then related to that for electronphoton scattering:

$$
\frac{d \sigma\left(e N \rightarrow e Z^{x} N^{\prime}\right)}{d\left(p_{e} \cdot k_{x}\right) d\left(p_{N} \cdot k_{x}\right)}=\left.\frac{\alpha}{\pi} \frac{d \sigma\left(e \gamma \rightarrow e Z^{x}\right)}{d\left(p_{e} \cdot k_{x}\right)}\right|_{q=q^{*}} \frac{\chi}{p_{N} \cdot k_{e}} .
$$

The two-to-two cross section is evaluated at the special kinematics $q=q^{*}$, where $\overrightarrow{q^{*}}$ is parallel to $\vec{p}_{e}-\vec{k}_{x}$. The effective flux $\chi$ contains all details of the target structure; it is also a function of the electron energy $E_{e}$ and vector mass $m_{x}$. We plot $\chi / Z^{2}$ in figure 6 for the E137 experiment [77] (see section 4.4), where the target atomic number $Z=13$ (compare figure 10 of ref. [19]). It is $\mathcal{O}(1-100)$ for $m_{x}<1 \mathrm{GeV}$, and drops sharply above that point. More details about this function are provided in appendix B.1.

Introducing $x_{e} \equiv E_{x} / E_{e}$, the total differential cross section is [19]

$$
\frac{d \sigma}{d x_{e} d \cos \theta_{x}}=8 \alpha^{3} \epsilon^{2} E_{e}^{2} x_{e} \chi \sqrt{1-\frac{m_{x}^{2}}{E_{e}^{2}}} f\left(x_{e}, m_{x}^{2}\right),
$$

where for later convenience we have defined

$$
f\left(x_{e}, m_{x}^{2}\right)=\frac{1-x_{e}+\frac{1}{2} x_{e}^{2}}{U^{2}}-\frac{\left(1-x_{e}\right) x_{e} m_{x}^{2}}{U^{3}}+\frac{\left(1-x_{e}\right)^{2} m_{x}^{4}}{U^{4}} .
$$

$U$ is the virtuality of the intermediate electron in the second diagram of figure 5 , and is a function of both $x_{e}$ and $\theta_{x}$ :

$$
U \approx E_{e}^{2} x_{e} \theta_{x}^{2}+m_{x}^{2} \frac{1-x_{e}}{x_{e}}+m_{e}^{2} x_{e}
$$




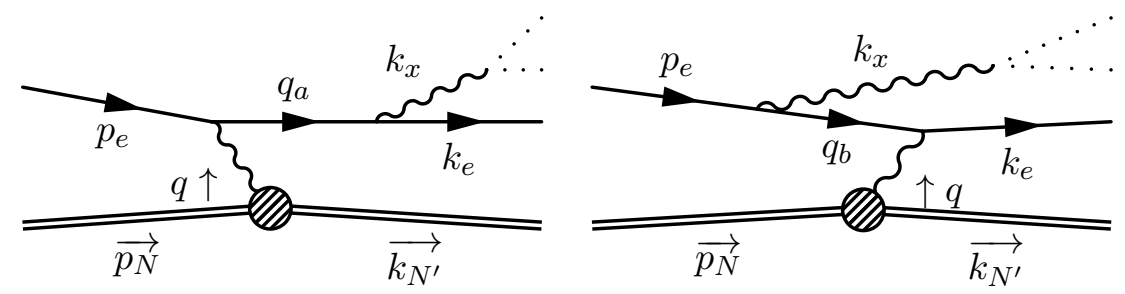

Figure 7. The two leading Feynman diagrams for production of off-shell massive vector in electronfixed target scattering. The dotted lines represent the on-shell hidden states; we integrate over the phase space of these particles in deriving eq. (4.7).

This cross section is sharply peaked at $\theta_{x} \approx 0$ and $x_{e} \approx 1$. Indeed, we see from eqs. (4.3) through (4.5) that there is a singularity in that limit that is only regulated by the electron mass. The WW approximation can be understood as focusing on these singular terms.

We integrate eq. (4.3) over the angular variable $\theta_{x}$. This gives us the differential cross section with respect to the electron energy,

$$
\frac{d \sigma}{d x_{e}}=4 \alpha^{3} \epsilon^{2} \chi \sqrt{1-\frac{m_{x}^{2}}{E_{e}^{2}}} \frac{1-x_{e}+\frac{1}{3} x_{e}^{2}}{m_{x}^{2} \frac{1-x_{e}}{x_{e}}+m_{e}^{2} x_{e}} .
$$

The total cross section for production of on-shell hidden vectors at the E137 and proposed JLab experiments are shown in figure 8 .

\subsection{Production cross section: off-shell vector}

We next consider the situation where we enter the hidden sector through an off-shell vector. This is the natural generalisation of the previous scenario. It is particularly relevant to Case B, where it is the only way to get a visible signal from the hidden sector; and Case A, where it is the only way to produce hidden states other than the hidden vector.

We focus on the production of two on-shell hidden states through the diagrams shown in figure 7. The dotted lines represent either $h_{1}^{x} Z^{x}$ (Higgsstrahlung channel), $h_{1}^{x} A^{x}$ (scalar channel) or $\chi_{1}^{x} \chi_{1,2}^{x}$ (fermion channel). Of these, the first is usually most important on kinematic grounds. Before fully generalising the WW approximation, we note that by integrating over the hidden state phase space and using the QED Ward identity, we can obtain a simple extension of eq. (4.2) (see ref. [22] and appendix B.2):

$$
\frac{d \sigma\left(e N \rightarrow e Z^{x(*)} N^{\prime}\right)}{d k_{x}^{2} d\left(p_{e} \cdot k_{x}\right) d\left(p_{N} \cdot k_{x}\right)}=\left.\left(\frac{1}{\pi} \frac{\sqrt{k_{x}^{2}} \Gamma_{\mathrm{HS}}\left(k_{x}^{2}\right)}{\left(k_{x}^{2}-m_{x}^{2}\right)^{2}}\right) \frac{\alpha}{\pi} \frac{d \sigma\left(e \gamma \rightarrow e Z^{x}\right)}{d\left(p_{e} \cdot k_{x}\right)}\right|_{q^{*}} \frac{\chi}{p_{N} \cdot k_{e}}
$$

This expression is valid under the conditions of eq. (4.1) with the replacement $m_{x} \rightarrow \sqrt{k_{x}^{2}}$. The partial width $\Gamma_{\mathrm{HS}}$ is for a vector of mass $m_{x}^{2}=k_{x}^{2}$ to decay to the relevant final state; explicit expressions are given in appendix B.3. Note that if we replace the numerator with a Breit-Wigner propagator and allow the vector to go on-shell, then in the narrow-width approximation the bracketed term in eq. (4.7) reduces to a delta function, reproducing eq. (4.2). We show the production cross sections for off-shell hidden vectors in figure 8 for two cases that are extremal among those we consider. 


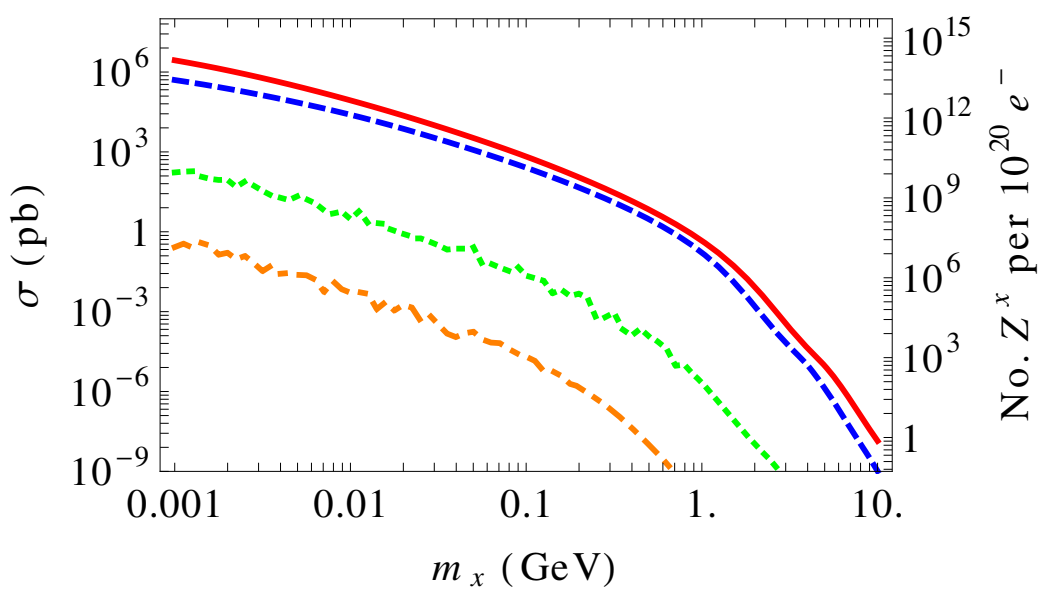

Figure 8. Production cross sections and hidden sector thick-target yields for $\epsilon=10^{-3}$ at electron beam dump experiments. The red, solid (blue, dashed) lines are for on-shell production at E137 (JLab). The orange, dot-dashed (green, dotted) lines are for off-shell production at E137 in the scalar (Higgsstrahlung) channels in Case A; these are the extremal off-shell cross sections for our model.

The full generalisation of the WW approximation is most conveniently expressed in terms of integrated matrix elements, due to non-trivial phase space factors. We denote the electron-target and electron-photon amplitudes by $\mathcal{M}_{e N}$ and $\mathcal{M}_{e \gamma}$ respectively, where the latter is evaluated at the special kinematics $q=q^{*}$. Then the WW approximation is

$$
\int d \Pi_{n+2} \frac{1}{2 N_{i}} \sum\left|\mathcal{M}_{e N}\right|^{2}=\int\left(\prod_{i=1}^{n} \frac{d^{3} k_{i}}{(2 \pi)^{3} 2 E_{i}}\right) \frac{1}{4} \sum\left|\mathcal{M}_{e \gamma}\right|^{2} \frac{M_{i}}{\left|\vec{p}_{e}-\vec{k}_{x}\right|} \frac{\alpha \chi}{t_{\mathrm{aux}}}
$$

Here, $M_{i}$ is the mass of the target, $N_{i}$ its internal degrees of freedom, the vector magnitude $\left|\vec{p}_{e}-\vec{k}_{x}\right|$ is evaluated in the lab frame, and $t_{\text {aux }} \equiv\left(p_{e} \cdot k_{X}-\frac{1}{2} m_{x}^{2}\right) /\left(E_{e}-E_{x}\right)$ is roughly the minimum momentum transfer between the electron and the target. There are $n$ hidden sector particles with momenta $k_{i}, i=1 \ldots n$, and $d \Pi_{l}$ represents the $l$-dimensional phase space integral including the delta function that enforces overall conservation of momentum. More details are presented in appendix B.2.

For the case $n=2$ of interest, the hidden sector kinematics may be expressed in terms of the momentum of the off-shell vector $k_{x}$, and $k_{\Delta} \equiv k_{1}-k_{2}$. Requiring that the final states be on-shell imposes

$$
\begin{aligned}
k_{\Delta}^{2} & =2 m_{1}^{2}+2 m_{2}^{2}-k_{x}^{2}, \\
k_{\Delta} \cdot k_{x} & =m_{1}^{2}-m_{2}^{2} .
\end{aligned}
$$

The first constraint can be used to fix $\left|\vec{k}_{\Delta}\right|$, and the second to fix the angle between $\vec{k}_{x}$ and $\vec{k}_{\Delta} . k_{\Delta}$ is then defined by $k_{\Delta}^{0}$ and $\phi_{\Delta}$, with the latter being the azimuthal angle for the vector $\vec{k}_{\Delta}$ relative to $\vec{k}_{x}$. The lab-frame cross sections we desire are, in the Higgsstrahlung 
channel:

$$
\begin{aligned}
\frac{\mathrm{d} \sigma}{\mathrm{d} k_{x}^{2} \mathrm{~d} k_{x}^{0} \mathrm{~d} \cos \theta_{x} \mathrm{~d} k_{\Delta}^{0} \mathrm{~d} \phi_{\Delta}}=\frac{\alpha^{3} \alpha_{x} \epsilon^{2} \chi}{4 \pi^{2}} & N_{H, a}^{2} \frac{1}{\left(k_{x}^{2}-m_{x}^{2}\right)^{2}} \\
\times & \times\left[\left(k_{x}^{2}-2 m_{h_{1}^{x}}^{2}+6 m_{x}^{2}\right) f\left(x_{e}, k_{x}^{2}\right)+2 \Omega\left(k_{\Delta}\right)\right],
\end{aligned}
$$

for the production of a pair of scalars:

$$
\begin{aligned}
\frac{\mathrm{d} \sigma}{\mathrm{d} k_{x}^{2} \mathrm{~d} k_{x}^{0} \mathrm{~d} \cos \theta_{x} \mathrm{~d} k_{\Delta}^{0} \mathrm{~d} \phi_{\Delta}}=\frac{\alpha^{3} \alpha_{x} \epsilon^{2} \chi}{4 \pi^{2}} & N_{S, a}^{2} \frac{1}{\left(k_{x}^{2}-m_{x}^{2}\right)^{2}} \\
& \times\left[\left(k_{x}^{2}-2 m_{1}^{2}-2 m_{2}^{2}\right) f\left(x_{e}, k_{x}^{2}\right)+2 \Omega\left(k_{\Delta}\right)\right],
\end{aligned}
$$

and for a pair of fermions

$$
\begin{aligned}
& \frac{\mathrm{d} \sigma}{\mathrm{d} k_{x}^{2} \mathrm{~d} k_{x}^{0} \mathrm{~d} \cos \theta_{x} \mathrm{~d} k_{\Delta}^{0} \mathrm{~d} \phi_{\Delta}}=\frac{\alpha^{3} \alpha_{x} \epsilon^{2} \chi}{2 \pi^{2}} \frac{1}{\left(k_{x}^{2}-m_{x}^{2}\right)^{2}} \\
& \times\left[\left(\left|N_{F, i j}\right|^{2} k_{x}^{2}-4 m_{1} m_{2}\left(\Re N_{F, i j}^{2}\right)\right) f\left(x_{e}, k_{x}^{2}\right)-2\left|N_{F, i j}\right|^{2} \Omega\left(k_{\Delta}\right)\right] .
\end{aligned}
$$

The various $N$ s are the combinations of mixing matrices that appear in the three-point vertices $X^{\mu}-X^{\nu}-h_{a}^{x}, X^{\mu}-h_{a}^{x}-A^{x}$ and $X^{\mu}-\chi_{i}^{x}-\chi_{j}^{x}$, respectively. In practice we need only consider $a=1$ and $i=1, j \in\{1,2\}$. They are explicitly given by

$$
\begin{aligned}
N_{H, a} & =\sin \zeta R_{1 a}+\cos \zeta, R_{2 a}, \\
N_{S, a} & =\cos \zeta R_{1 a}-\sin \zeta R_{2 a}, \\
N_{F, i j} & =P_{i 1} P_{j 1}^{*}-P_{i 2} P_{j 2}^{*} .
\end{aligned}
$$

$R$ is the matrix that relates the real hidden scalar mass and gauge bases, as defined in eq. (A.6). $P$ is the equivalent matrix for the hidden fermions [26]. We have used $x_{e}$ and $U$ defined as in the on-shell case with $m_{x}^{2} \rightarrow k_{x}^{2}$. The $k_{\Delta}$-dependence comes through the function

$$
\begin{aligned}
\Omega\left(k_{\Delta}\right)= & \frac{\left(1-x_{e}\right)}{U^{3}}\left(m_{1}^{2}-m_{2}^{2}\right)\left(\left(1-x_{e}\right) p_{e} \cdot k_{\Delta}+\left(p_{e}-q\right) \cdot k_{\Delta}-\left(m_{1}^{2}-m_{2}^{2}\right)\right) \\
& -\frac{\left(1-x_{e}\right)^{2}}{U^{4}} k_{x}^{2}\left(\left(p_{e} \cdot k_{\Delta}\right)^{2}+\left(\left(p_{e}-q\right) \cdot k_{\Delta}\right)^{2}-\left(m_{1}^{2}-m_{2}^{2}\right)\left(2 p_{e}-q\right) \cdot k_{\Delta}\right) .
\end{aligned}
$$

In the limit that the WW approximation is valid, $\left(p_{e}-q\right) \cdot k_{\Delta} \approx p_{e} \cdot k_{\Delta}$; while

$$
p_{e} \cdot k_{\Delta}=E_{e}\left(k_{\Delta}^{0}-\left|\vec{k}_{\Delta}\right|\left(\cos \theta_{x} \cos \theta_{\Delta}-\sin \theta_{x} \sin \theta_{\Delta} \cos \phi_{\Delta}\right)\right),
$$

with $\theta_{\Delta}$ the angle between $\vec{k}_{x}$ and $\vec{k}_{\Delta}$.

\subsection{Expected number of signal events}

In the previous two sections we gave the differential cross sections for the production of hidden sector states at beam dump experiments. Here we relate those expressions to the 
actual experimental observables, specifically the number of events seen in the detectors. We first relate the cross sections to the number of hidden sector particles actually produced, and then discuss the acceptance factors that give the number of signals per hidden sector event.

For a fixed target experiment, we must account for interactions throughout the full depth of the target material. This must also include energy loss from the electron beam as it travels through the target. In particular, the electron energy in eq. (4.3) and similar expressions satisfies $E_{e} \leq E_{0}$, the incident beam energy. This effect is handled by convolving the production cross section with an attenuation function. We have the following expression for the distribution of the number of hidden vectors produced:

$$
\frac{d N}{d x_{0}}=N_{e} \frac{N_{0} X_{0}}{A} \int_{E_{x}+m_{e}}^{E_{0}} d E_{e} \int_{0}^{T} d s I\left(E_{0}, E_{e}, s\right) \frac{E_{0}}{E_{e}} \frac{d \sigma}{d x_{e}} .
$$

Here, $N_{e}$ is the total number of electrons delivered to the target; $N_{0}$ is Avogadro's number; $A$ is the atomic number and $X_{0}$ the unit radiation length $\left(\mathrm{M} / \mathrm{L}^{3}\right)$ of the target material; $s$ is the depth in radiation lengths within the target, of total thickness $T$; and $x_{0, e}=E_{x} / E_{0, e}$.

The attenuation function of eq. (4.16) gives the energy distribution of an initiallly monochromatic electron beam after travelling $s$ radiation lengths through the target material. We follow refs. $[67,78]$ in taking $^{2}$

$$
I\left(E_{0}, E_{e}, s\right)=\frac{1}{E_{0}} \frac{\left[\ln \left(\frac{E_{0}}{E_{e}}\right)\right]^{b s-1}}{\Gamma(b s)},
$$

with $\Gamma$ the Gamma function and $b=\frac{4}{3}$. Since $\sigma \propto E_{e}^{2}$ (see eq. (4.3)), production is dominated from electrons with the initial beam energy; and from $I$, this mostly occurs in the first few radiation lengths of the target.

We define an average acceptance number that is the ratio of the number of signal events to the total number of hidden sector events computed above. There are two steps in deriving these factors. First, we need to infer the kinematic distributions for the stable or metastable states from the differential cross sections of sections 4.1 and 4.2. Next we must determine either what fraction of metastable states' decay products will give visible signals; or what fraction of hidden sector particles will scatter in the detector, depending on the nature of the experiment.

We compute these using a simple Monte Carlo simulation. First we generate events drawn from the appropriate one of the distributions in eqs. (4.3), (4.10), (4.11) and (4.12). For this purpose, an event is defined by the four-momenta of one or two on-shell hidden states. When the hidden vector has on-shell decays to the final states of interest, we generate its kinematics using eq. (4.3); otherwise we use the appropriate one of eqs. (4.10), (4.11) and (4.12) and generate both hidden states' four-momenta.

For on-shell processes, we select the $Z^{x}$ energy fraction $x_{e}$ from eq. (4.6) first, then the polar angle $\theta_{x}$ given $x_{e}$ from eq. (4.3). To select the variables, we generate candidate values randomly within the physically allowed range, then keep those values with probability given

\footnotetext{
${ }^{2}$ For an alternate but similar parameterisation, see refs. [19, 22].
} 
by the ratio of the kinematic distribution to its maximum value. For example, a candidate value for $x_{e}$ is kept with probability

$$
P^{\text {keep }}=\frac{d \sigma}{d x_{e}}\left(x_{e}^{\text {cand }}\right) / \frac{d \sigma}{d x_{e}}\left(x_{e}^{\max }\right) .
$$

When choosing to keep or reject $\theta_{x}$, we maximise eq. (4.3) for fixed $x_{e}$. The azimuthal angle is selected randomly, as the spin-averaged cross sections do not depend on it.

For off-shell processes, we first generate the hidden vector four-momenta, then use the appropriate one of eqs. (4.10), (4.11) and (4.12) to simultaneously generate the remaining two non-trivial kinematic variables $k_{\Delta}^{0}$ and $\phi_{\Delta}$. Generating $k_{x}$ is done as for on-shell processes, except we must first select the unconstrained $k_{x}^{2}$ using the distribution (derivable from eqs. (4.2) and (4.7))

$$
P\left(k_{x}^{2}\right) \propto \frac{\sqrt{k_{x}^{2}} \Gamma_{\mathrm{HS}}\left(k_{x}^{2}\right)}{\left(k_{x}^{2}-m_{x}^{2}\right)^{2}} \sigma\left(k_{x}^{2}\right) .
$$

Again, there is no dependence on the $k_{x}$ azimuthal angle, and other kinematics are constrained by requiring final states by on-shell, eq. (4.9).

We then decay through the hidden sector till we reach the stable and metastable states. In doing so we use the narrow width approximation, treating all decays as prompt and isotropic in the parent rest frame. We also track all branching ratios as necessary, though in most cases these are trivial. This effectively gives us the (meta-)stable particle kinematics.

The subsequent steps then depend on the nature of the experiment. For searches based on visible sector particles, there is another branching ratio; the experiments we will discuss triggered only on electrons, suppressing sensitivity for hidden sector masses above the muon threshold. The acceptance factor is the product of all relevant branching ratios, the probability that the metastable state decays within the decay volume, and that its visible daughter particles hit the detector with sufficient energy to pass the threshold. We compute this on an event-by-event basis. The decay probability is

$$
\begin{aligned}
P_{\mathrm{dec}} & =\exp \left[-\frac{L_{\mathrm{sh}}}{\gamma v_{z} \tau}\right]\left(1-\exp \left[-\frac{L_{\mathrm{dec}}}{\gamma v_{z} \tau}\right]\right) \\
& =\exp \left[-\frac{L_{\mathrm{sh}}}{\tau} \frac{m}{p_{z}}\right]\left(1-\exp \left[-\frac{L_{\mathrm{dec}}}{\tau} \frac{m}{p_{z}}\right]\right),
\end{aligned}
$$

with $L_{\mathrm{sh}}$ and $L_{\mathrm{dec}}$ as shown in figure $3, v_{z}$ and $p_{z}$ are the parent's velocity and momentum along the beam direction, $m$ is its mass and $\tau$ its lifetime. The angular acceptance probability is found by extending our Monte Carlo, decaying the metastable states within the decay volume and counting what fraction of daughter particles pass the experimental cuts. To this end, we model the experiments as cylinders of radius $r_{\text {Acc }}$ with hard energy threshold cuts at $E_{\mathrm{thr}}$. The final number of signal events is

$$
N_{s i g}=N \times \frac{1}{E V} \sum_{i \in H I T} \operatorname{Br}_{\text {tot }}^{i} \times P_{\mathrm{dec}}^{i},
$$

where $E V$ is the total number of events we generate, and $H I T$ the set of metastable states with daughter particles observed in the detector. 
For the proposed search of ref. [22] based on invisible particles, the remaining factors are much simpler. The angular acceptance is directly found from the kinematic distribution of metastable states, asking what fraction strike the detector (including, where necessary, a survival probability). This is multiplied by the event-by-event probability that the hidden sector state scatter within the detector:

$$
P_{s c a t}=\frac{\rho}{m_{N}} L_{d} \sigma_{\chi N}
$$

with $\rho$ the target density, $m_{N}$ the nucleon mass, $L_{d}=1 \mathrm{~m}$ the detector depth as in figure 4 and $\sigma_{\chi N}$ the total hidden sector-nucleon scattering cross section summed over final states. This assumes that multiple scattering and attenuation of the dark sector beam is negligible $\left(P_{\text {scat }} \ll 1\right)$. Scattering to different hidden sector states often dominates due to mixing matrix suppression of the elastic process. For this reason we neglect scattering from electrons, where the inelastic channels are usually kinematically forbidden.

Full details on the differential scattering cross sections are given in appendix C. When the incident particle is relativistic we may approximate [22]

$$
\sigma_{\chi N} \sim 4 \pi \epsilon^{2} \alpha \alpha_{x} N_{I}^{2} \frac{Z}{A} \frac{1}{\mu^{2}}
$$

with

$$
\frac{1}{\mu^{2}}= \begin{cases}\left(Q_{\max }^{2}-Q_{\min }^{2}\right) / m_{x}^{4} & m_{x}^{2} \gg Q_{\max }^{2}, \\ 1 / m_{x}^{2} & Q_{\min }^{2} \lesssim m_{x}^{2} \lesssim Q_{\max }^{2}, \\ 1 / Q_{\min }^{2} & m_{x}^{2} \ll Q_{\min }^{2} .\end{cases}
$$

$Q^{2}$ is the momentum transfer in the scattering process, with $Q_{\min } \sim 140 \mathrm{MeV}$ and $Q_{\max } \sim$ $1 \mathrm{GeV}$ (for the JLab experiment). $N_{I}$ is the mixing matrix factors for the three-point coupling of the hidden sector state to $X_{\mu}$, summed over possible final states. In the case when all hidden sector states are accessible, we have

$$
N_{I}^{2}= \begin{cases}1 & \text { for } A^{x}, h_{a}^{x}, \\ \left|P_{i 1}\right|^{2}+\left|P_{i 2}\right|^{2} & \text { for } \chi_{i}^{x} .\end{cases}
$$

We take the signal yield as

$$
N_{\text {sig }}=N \times \frac{1}{E V} \sum_{i \in H I T} \operatorname{Br}_{\text {tot }}^{i} \times P_{\text {scat }}^{i} \times P_{\text {surv }}^{i},
$$

with $E V$ the number of simulated particles, HIT the set of hidden particles with trajectories intersecting the detector, and $P_{\text {surv }}$ the probability of that state not decaying before passing through it.

\subsection{Current and prospective experiments}

Let us first briefly review the experiments that have been used or proposed to set limits on low energy hidden sectors. 


\begin{tabular}{|ccrrrrrrr|}
\hline Experiment & Target & $E_{0}$ & $N_{e}$ & $L_{\text {sh }}$ & $L_{\text {dec }}$ & $E_{\text {thr }}$ & $r_{\text {Acc }}$ & $N_{95 \%}$ \\
\hline E137 & $\mathrm{Al}$ & 20 & $1.87 \times 10^{20}$ & 179 & 204 & 2 & 1.5 & 3 \\
E141 & $\mathrm{W}$ & 9 & $2 \times 10^{15}$ & 0.12 & 35 & 4.5 & 0.0375 & 3419 \\
E774 & $\mathrm{W}$ & 275 & $5.2 \times 10^{9}$ & 0.3 & 2 & 27.5 & 0.1 & 18 \\
KEK & $\mathrm{W}$ & 2.5 & $1.69 \times 10^{17}$ & 2.4 & 2.2 & 0.1 & 0.047 & 3 \\
Orsay & $\mathrm{W}$ & 1.6 & $2 \times 10^{16}$ & 1 & 2 & 0.75 & 0.15 & 3 \\
JLab & $\mathrm{Al}$ & 12 & $10^{20}$ & 10 & & & 1 & \\
\hline
\end{tabular}

Table 2. Parameters of the electron beam dump experiments we consider. The parameters $E_{0}$ and $E_{\text {thr }}$ are in $\mathrm{GeV}$, while $L_{\mathrm{sh}}, L_{\mathrm{dec}}$ and $r_{\mathrm{Acc}}$ are in metres.

Previous beam dumps. Several previous beam dump experiments have been used to set limits on hidden vectors decaying directly to leptons [19, 67]. These include E137 [79] and E141 [77] at SLAC, E774 [80] at Fermilab, KEK [81], and Orsay [82]. We use the experimental parameters as given in table 2. The energy thresholds are taken from the relevant experimental papers; they were usually ignored in previous work, as they are trivial for searches based on $Z^{x} \rightarrow e^{+} e^{-}$. For our more complex decay chains they must be included. Note that all these experiments were only sensititve to $e^{ \pm}$, except for E141 which was only sensitive to positrons. We demand that the number of expected events be less than $N_{95 \%}$.

Present and future visible searches. A number of experiments are currently running or have recently been proposed to search for low energy hidden sectors. This includes MAMI [68], APEX [69, 71], HPS [73, 74], a proposal at the CERN SPS [83, 84], and DarkLight [72]. Regretably, these experiments do not set any limits on our model beyond those that apply in Case A. For APEX, MAMI and HPS, a crucial tool used to discriminate signal from background is the requirement that the observed electron-positron pair have combined energy equal to the beam energy. This is based on the sharp peak in the cross sections at $x_{e} \approx 1$ in eq. (4.3). However, if the hidden vector decays to hidden sector particles - or is not produced on-shell - then the initial beam energy will be distributed among a larger number of particles. Hidden sector events in our model will then not pass the signal cuts.

The proposed CERN SPS search uses different discriminators, but with a similar philosophy. Calorimeters will be positioned before and after the decay volume, and the total energy in them must sum to the incident beam energy. For the small scale of the decay volume $(\sim 5 \mathrm{~m})$ the hidden Higgs is effectively stable, so all non-trivial hidden sector events will involve irreducible missing energy from either $h_{1}^{x}$ or $\chi_{1}^{x}$. We thus expect that no events will pass the experimental cuts. It is also planned to search for invisible decays [84]; this likely would have some sensitivity to cases B-D, but we defer a full investigation of this for a future paper.

For DarkLight, the situation is slightly different. DarkLight uses a peak in the electronpositron invariant mass spectrum to discriminate the signal. In our model, the pseudoscalar $A^{x}$ and fermion $\chi_{2}^{x}$ have three-body decays, so will not pass this cut. The scalar $h_{1}^{x}$ will 
pass the cut; however, DarkLight requires a relatively prompt decay. In the mass range to which DarkLight is senstive (less than $100 \mathrm{MeV}$ ), the scalar has a decay length of $\gtrsim 10 \mathrm{~m}$ for $\epsilon \lesssim 0.1$. It follows that there will be no sensitivity below this kinetic mixing.

JLab inivisible search. The proposed search at JLab [22] would have the experimental parameters listed in table 2. Note that for this experiment, the proposed detector would have a square cross section so $r_{\text {Acc }}$ should be interpreted as the side of that square. We follow the original proposal in taking three different choices for sensitivity, corresponding to different possibilities for background subtraction. In order from least to most optimistic, we consider sensitivity to 20000,1000 and 40 events respectively.

\subsection{Limits}

We now present current and prospective limits for our four benchmark scenarios. For clarity, we show only limits from electron beam dump experiments, as well as from the electron and muon anomalous magnetic moments (to aid in comparisons with other results). For composite plots showing all limits we find, see section 7 . We show limits in the $m_{x}-\epsilon$ plane for benchmarks $\mathrm{A}$ and $\mathrm{B}$ in figure 9, and benchmarks $\mathrm{C}$ and $\mathrm{D}$ in figure 10. For benchmarks A through $\mathrm{C}$, we also show the mass of the lightest scalar $h_{1}^{x}$ on the upper horizontal axis; for benchmark $\mathrm{D}$, we show the mass of the lightest fermion $\chi_{1}^{x}$.

The general features of all exclusion plots from visible searches are straightforward. At large kinetic mixing, the exclusion contours are set when the lifetime of the metastable particle becomes too short. They are approximately contours of $\epsilon^{2} m_{x}$, except near particle thresholds. At small kinetic mixing, the boundary is mainly set when the production cross section becomes too small, which is only weakly dependent on mass. Finally, there is a lower limit on all these searches when the long-lived particle can no longer decay to electrons. While decays to photons might fake electrons in the experimental detectors, the lifetimes are highly suppressed with the result that no limits from these searches can be set.

Case A. This benchmark corresponds to the previously-studied case, where the hidden vector may only decay to the visible sector. We therefore include past limits for electron beam dumps taken from ref. [67] in figure 9. We find no new limits beyond those. Additional constraints could in principle arise from the production of hidden sector scalars or fermions through an off-shell vector; but a combination of kinematic and coupling suppressions result in this not being the case. The strongest such limits we find come from long-lived $h_{1}^{x}$ decays at the E137 experiment. These are shown as the red shaded region in figure 9, but are weaker than the current constraints from $a_{e}{ }^{3}$

Case B. Similarly to Case A, there are no new limits from previous experiments. The limits from long-lived scalars that would exist are comparable to those in Case A, and inferior to those from $a_{e}$. However, we now have promising prospective limits from the JLab search. Even in the most pessimistic background scenario, we can extend the exclusion contours to exclude much of the region preferred by $a_{\mu}$. This is unsurprising; the vector here decays invisibly to the stable $\chi_{1}^{x} \chi_{1}^{x}$ final state, precisely the motivating case for that

\footnotetext{
${ }^{3}$ This was not the case for the previous limits $[16,85]$.
} 

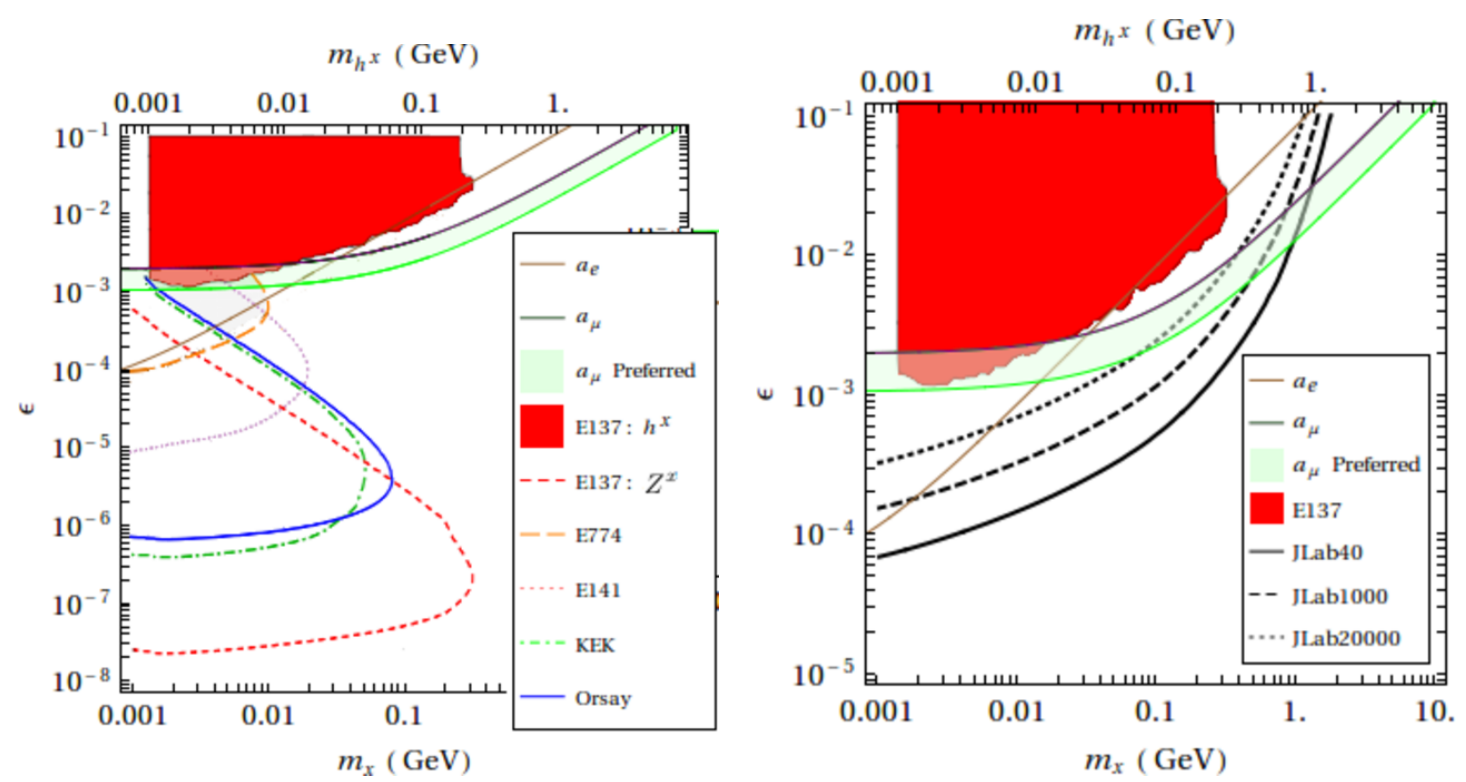

Figure 9. Exclusion plots from electron beam dumps for benchmark slopes A (left) and B (right). The constraints and preferred region from the electron and muon $g-2$ are shown for scale. For A, the unshaded contours are prior limits from $Z^{x} \rightarrow e^{+} e^{-}$, and the solid red region is the new limits from $h_{1}^{x} \rightarrow e^{+} e^{-}$at the E137 experiment. For B, the solid region shows the same $h_{1}^{x} \rightarrow e^{+} e^{-}$ limit, and the thicker contours mark 40,1000 and 20000 expected $\chi_{1}^{x}$ scattering events at the JLab search. See the text for more details.

search. The limits here are not directly comparable to the plots of refs. [22, 24]: that work fixed the mass of the stable hidden particle and varied the vector mass, while we vary both with a fixed mass ratio.

Case C. This benchmark offers a number of interesting new and prospective limits, that go beyond the constraints from $a_{e}$ and $a_{\mu}$. The improved reach is because we can produce unstable hidden sector states through an on-shell hidden vector. The phenomenology is richer because the vector decays to two particles, $h_{1}^{x}$ and $A^{x}$, that are both potentially long-lived.

We first address the limits from searches for visible decays. In figure 10 we only show the limits from E137; the limits from the other experiments listed in table 2 are strictly inferior. We show separately the limits from the scalar (red) and pseudoscalar (blue) decays. The scalar limits are sensitive to the Higgs mixing induced from $D$-terms as discussed in section A.3. The limits with (without) this mixing are shown in darker red with solid boundary (lighter red with dashed boundary), and labelled "SUSY" ("SM") in the key. The two cases are equivalent below the muon threshold, as the mass mixing is unimportant there (see figure 25). Above the muon threshold, the limits with the mixing are weaker due both to the hidden scalar decaying too quickly and the branching ratio to electrons is being more strongly suppressed.

The limits from pseudoscalar decays extend to lower kinetic mixings than those from the scalar decays. This is because the $A^{x}$ width is less suppressed than the $h_{1}^{x}$, so smaller 

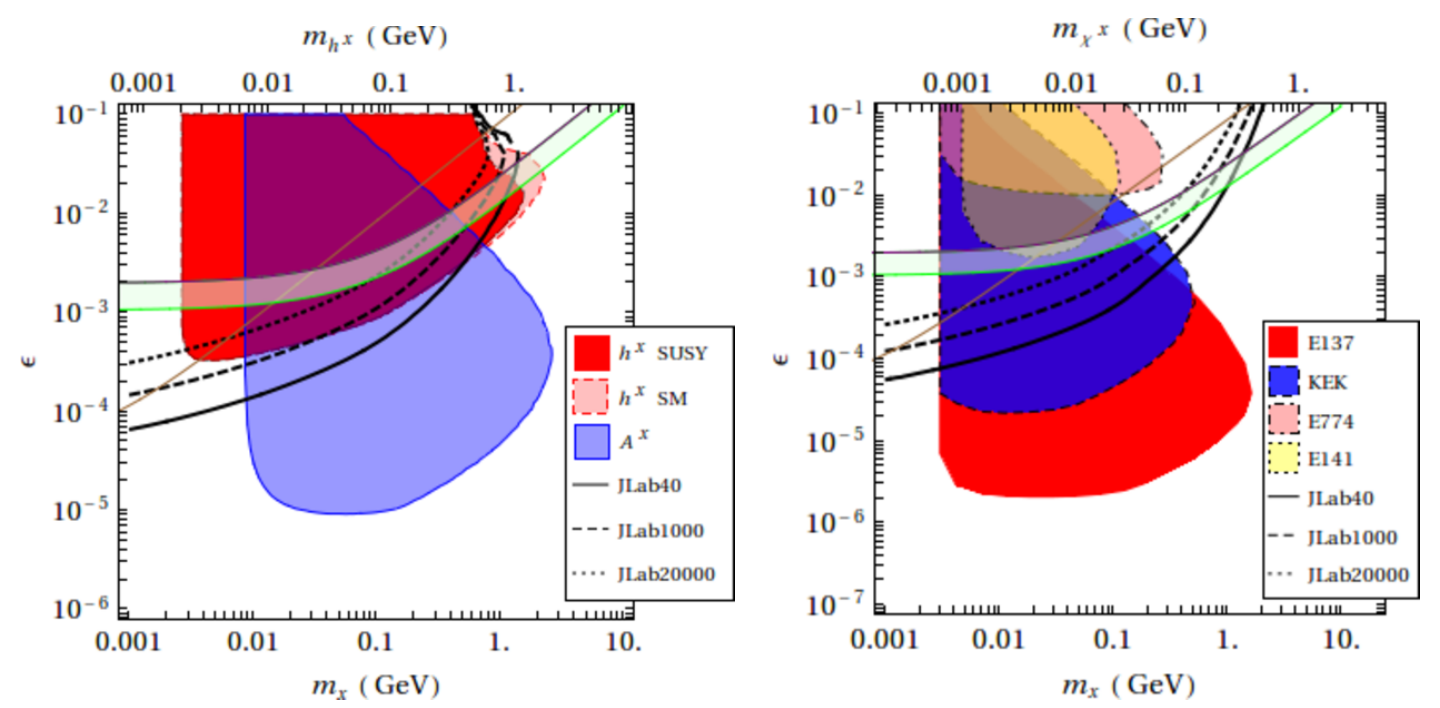

Figure 10. Exclusion plots from electron beam dumps for benchmark slopes C (left) and D (right). The limits from the electron and muon $g-2$ are shown with the same notation as in figure 9 , but are omitted from the keys for space. For C, the red regions denote E137 limits from $h_{1}^{x} \rightarrow e^{+} e^{-}$and the blue region from $A^{x} \rightarrow h_{1}^{x} e^{+} e^{-}$; the solid contours denote the expected number of $h_{1}^{x}$ scattering events at JLab. For D, the shaded regions are limits from $\chi_{2}^{x} \rightarrow \chi_{1}^{x} e^{+} e^{-}$at different experiments, and the solid contours the total number of expected $\chi_{1}^{x}$ and $\chi_{2}^{x}$ scattering events at JLab. See the text for more details.

values of $\epsilon$ are needed to have macroscopic decay lengths. There are two regions where the scalar limits are superior. At low mass, the threshold for the decay $A^{x} \rightarrow h_{1}^{x} e^{+} e^{-}$is crossed before that for $h_{1}^{x} \rightarrow e^{+} e^{-}$; while at high mass and moderate $\epsilon$, the pseudoscalar is too short-lived to produce an observable signal. Additionally, the limits from the pseudoscalar are less generic for two reasons. First, it is possible that the decay $A^{x} \rightarrow \chi_{1}^{x} \chi_{1}^{x}$ may be allowed and the corresponding scalar decay forbidden. Second, if CP is violated in the hidden sector and we choose a value of $\tan \zeta$ slightly closer to 1 , the decay $A^{x} \rightarrow h_{1}^{x} h_{1}^{x}$ may become relevant.

Finally, we have the prospective limits from the JLab invisible search. These limits are dominated from the scattering of the scalar $h_{1}^{x}$ in the detector. As such, while these limits are generically weaker than the pseudoscalar limits from E137, they might still be relevant if the latter are evaded as discussed above.

Case D. This benchmark has non-trivial limits from both visible and invisible searches. Both come from production of an on-shell vector that decays to $\chi_{2}^{x} \chi_{1}^{x}$. Visible searches look for the decay $\chi_{2}^{x} \rightarrow \chi_{1}^{x} e^{+} e^{-}$, suppressed for the same reasons (phase space and $\epsilon^{2}$ ) as the pseudoscalar in Case C. This slope is the only one of our four where visible searches other than E137 set limits, though that experiment still gives the strongest constraints. Of the five experiments in table 2, the limits from the Orsay experiment are strictly inferior to those from the KEK experiment, so we do not show them in figure 10.

Invisible searches for this benchmark are most sensitive to scattering of the $\chi_{1}^{x}$ in the detector. Of the two vector decay modes to the lightest fermion, the $\chi_{2}^{x} \chi_{1}^{x}$ channel dominates due to its larger branching ratio. While there remains a region of parameter 


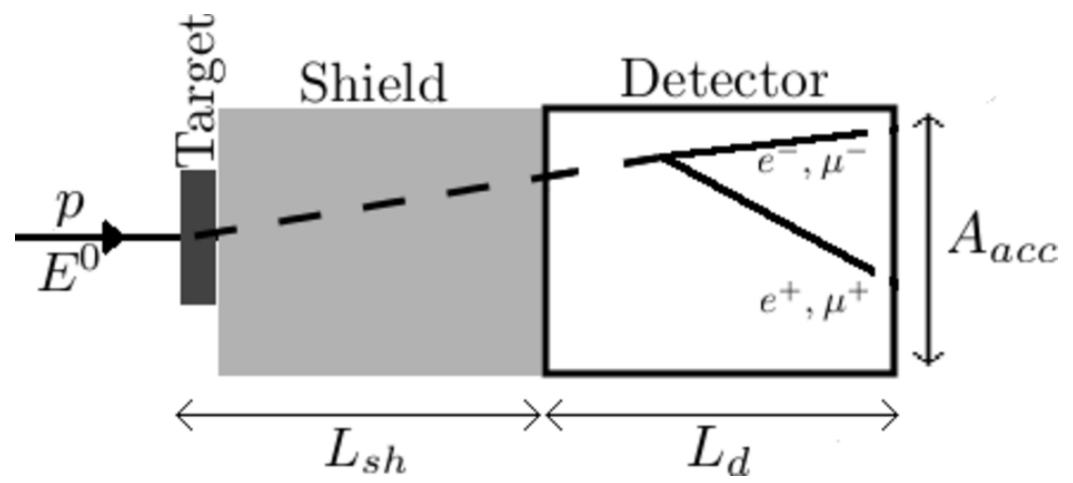

Figure 11. Experimental configuration for hadronic beam dump searches for visible decays. Note that the detector may be on the beam axis (as shown here) or off it.

space where $a_{\mu}$ can be explained that is not excluded by visible searches, it can be excluded by the JLab search.

\section{$5 \quad$ Hadronic fixed target experiments}

Hadronic fixed target experiments can also be used to constrain low-energy hidden sectors $[20,21,25,86-90]$. This is particularly relevant for hidden sectors with GeV-scale masses, where most constraints from electron experiments are limited by kinematics. As with electron beam dumps, most previous studies assumed the hidden vector either decays directly to the SM or to stable invisble particles. When long-lived scalars were considered $[20,25]$, they were required to decay only through hidden vector loops. The possible limits from hidden sector pseudoscalars and fermions have not been studied.

The searches for visible particles discussed in this section have the general configuration shown in figure 11. This is similar to figure 3, but the decay volume and detector are combined. Searches for the scattering of invisible particles have the same configuration of figure 4, with an incident beam of protons rather than electrons, and some of the experiments we consider placed the detector off the beam axis.

We discuss production of hidden sectors at these experiments in section 5.1 and the calculation of acceptances in section 5.2. This includes review of material covered elsewhere, both for completeness, and to extend some expressions for our more general hidden sector. In section 5.3 we discuss relevant experiments. The reader who is mainly interested in results should skip to section 5.4, where we present and discuss the exclusions we find for our four benchmark slopes.

\subsection{Production}

Hidden sector production in these experiments can roughly be divided into two domains [20], according to the hidden sector mass scale. If the hidden states have masses below $\Lambda_{Q C D}$, then production will mainly occur through the decay of mesons and baryons produced in the initial collision; we term this the "low mass" region. In the complementary "high mass" region, we can resolve the quark content of the proton and produce an $s$-channel hidden vector through $q \bar{q}$ fusion. 


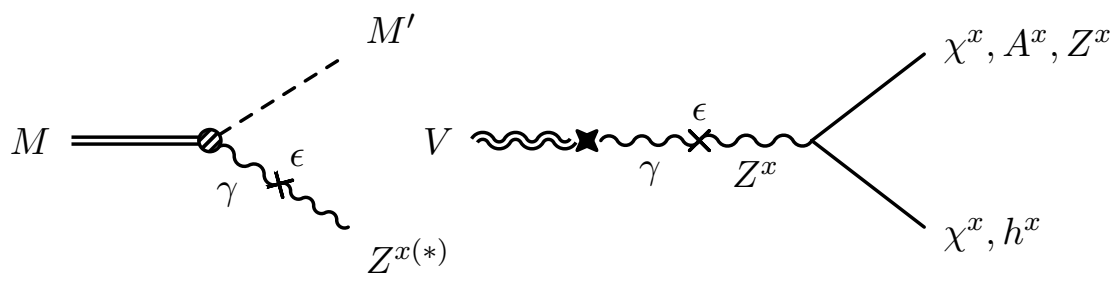

Figure 12. Hadron decays to the hidden sector. Left: for any SM decay $M \rightarrow M^{\prime}+\gamma$, there is a hidden sector companion decay $M \rightarrow M^{\prime}+Z^{x(*)}$ through the vector kinetic mixing. Right: a vector meson mixes with the hidden vector through their mutual mixing with the photon, allowing it to decay to two hidden sector particles.

The low mass regime was previously considered in ref. [20]. We extend some of their results to include new decay modes, in particular eq. (5.3). Production of hidden sector states occurs primarily through the secondary decay of hadrons produced at the interaction point. These decays can proceed either through kinetic mixing with the hadron decay product or with the hadron itself. In the former class, for any hadron $M$ with an electromagnetic decay of the form $M \rightarrow \gamma+\ldots$, there will be a companion decay $M \rightarrow Z^{x(*)}+\ldots$ In the second, a vector meson can mix with the $Z^{x}$ e.g. through a mutual mixing with the photon, allowing it to decay into the hidden sector. These possibilities are illustrated in figure 12 .

The most important example of the first category is the diphoton decay of a neutral pion. This has maximal branching ratio, and pions are produced in abundance in any hadron experiment. To extend the reach above the pion mass, we also consider the diphoton decays of the heavier pseudoscalars $\eta, \eta^{\prime}$; and the baryonic decay $\Delta \rightarrow N \gamma$. When the hidden vector is on-shell, its production has branching ratio

$$
\begin{aligned}
\operatorname{Br}\left(M \rightarrow \gamma Z^{x}\right) & =2 \epsilon^{2}\left(1-\frac{m_{x}^{2}}{m_{M}^{2}}\right)^{3} \operatorname{Br}(M \rightarrow \gamma \gamma), \\
\left(\Delta \rightarrow N Z^{x}\right) & =\epsilon^{2}\left(1-\frac{m_{x}^{2}}{\left(m_{\Delta}-m_{N}\right)^{2}}\right)^{3 / 2} \operatorname{Br}(\Delta \rightarrow N \gamma) .
\end{aligned}
$$

This assumes that the additional decay does not change the total width, a good approximation for $\epsilon \lesssim 0.1$. We also have the possibility of decays involving an off-shell hidden vector; these have branching ratio

$$
\operatorname{Br}_{\mathrm{HS}}\left(M \rightarrow M^{\prime} Z^{x *}\right)=\frac{1}{\pi} \epsilon^{2} \int_{m_{\mathrm{HS}}^{2}}^{m_{M}^{2}} d q^{2} \frac{\sqrt{q^{2}} \Gamma_{\mathrm{HS}}\left(q^{2}\right)}{\left(q^{2}-m_{x}^{2}\right)^{2}} \operatorname{Br}_{2}\left(m_{x}^{2}=q^{2}\right),
$$

where $\Gamma_{\mathrm{HS}}$ is the partial width from appendix B.3 appropriate to the hidden final states; $m_{\mathrm{HS}}$ is the sum of those masses; and $\mathrm{Br}_{2}$ refers to the on-shell expression from eq. (5.1) with $m_{x}^{2}$ replaced by $q^{2}$.

The second class of hadronic decays into the hidden sector involve the mixing of a vector meson with the hidden vector. This is exemplified by the $\rho$ meson. As is wellknown, the $\rho$ mixes with the photon; successive insertions of the $\rho-\gamma$ and $\gamma-X^{\mu}$ mixings then lead to a $\rho$-hidden vector mixing, which allows $\rho$ to decay to hidden states. This leads 

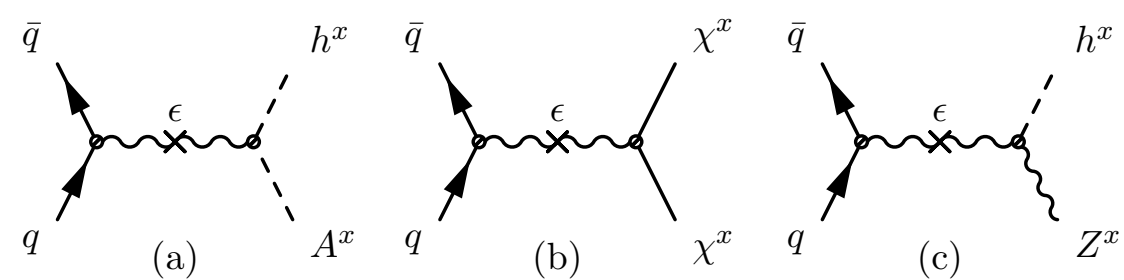

Figure 13. Feynman diagrams for the three parton-level processes that produce two hidden sector particles at hadronic beam dumps in the high mass regime. All proceed through an s-channel hidden vector to produce hidden scalars (a), fermions (b) or a scalar-vector pair (c).

to a $\rho$ branching ratio

$$
\operatorname{Br}(\rho \rightarrow H S)=\frac{12 \epsilon^{2}}{\alpha} \operatorname{Br}\left(\rho \rightarrow e^{+} e^{-}\right) \frac{m_{\rho}^{3} \Gamma_{\mathrm{HS}}\left(m_{\rho}^{2}\right)}{\left(m_{\rho}^{2}-m_{x}^{2}\right)^{2}+m_{x}^{2} \Gamma_{x}^{2}} .
$$

The propagator is regulated by the total hidden vector width $\Gamma_{x}$, which is relevant as $m_{x} \rightarrow m_{\rho}$. Analogous results hold for other neutral vector mesons.

The total number of hidden particles produced is proportional to the number of different hadron species produced. When possible, we use measurements or estimates of this from the experimental collaborations themselves. Otherwise, we model the production as dominantly in the first interaction length at full beam energy. This lets us relate the hadron production cross sections and yields using a simplified version of eq. (4.16):

$$
N_{M} \approx \sigma_{N} \frac{N_{0} X^{\mathrm{nuc}}}{A} N_{p}
$$

where $N_{p}$ is the total number of protons on target, $X^{\text {nuc }}$ is the nuclear interaction length, and $\sigma_{N}$ is the per-nucleus cross section. We then have $N_{X}=\operatorname{Br}(M \rightarrow X) \times N_{M}$, summed over hadron flavour $M$.

In the high mass regime, we can produce two-body hidden sector final states through either an on- or off-shell vector, through the processes shown in figure 13. We have the scalar, fermion and (for the intermediate vector off-shell) scalar-vector final states. If the vector is on-shell, the tree-level partonic cross section is

$$
\hat{\sigma}_{q}(\hat{s})=\frac{4 \pi^{2} \alpha}{3}\left(\epsilon Q_{q}\right)^{2} \delta\left(\hat{s}-m_{x}^{2}\right) \mathrm{Br}_{x},
$$

with $\mathrm{Br}_{x}$ the hidden vector branching ratio to the final state of interest. If the vector is off-shell, the equivalent expression may be written in terms of the vector partial widths from appendix B.3:

$$
\hat{\sigma}_{q}(\hat{s})=\frac{4 \pi \alpha}{3}\left(\epsilon Q_{q}\right)^{2} \frac{\sqrt{\hat{s}} \Gamma_{\mathrm{HS}}(\hat{s})}{\left(\hat{s}-m_{x}^{2}\right)^{2}}
$$

with $\hat{s}$ the partonic center of mass energy. We find the per-nucleon cross sections by convolving eqs. (5.5) and (5.6) with the CTEQ 10 parton density functions (pdfs) [91] in the usual way,

$$
\sigma_{p, n}=\int d x_{1} d x_{2} \sum_{q} f_{q \mid p, n}\left(x_{1}\right) f_{\bar{q} \mid p, n}\left(x_{2}\right) \hat{\sigma}_{q}\left(x_{1} x_{2} s\right) .
$$



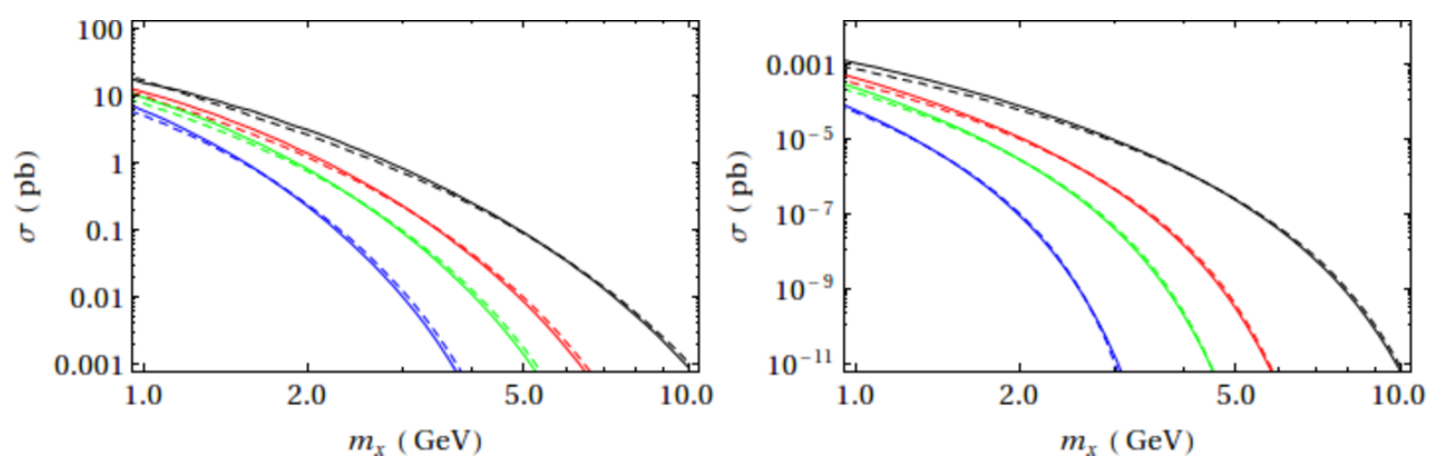

Figure 14. Cross sections $\sigma_{p}$ at hadronic beam dumps. Left: on-shell production in case C. Right: Higgsstrahlung production in case A. Solid (dashed) lines are at tree level $\left(\alpha_{S}\right)$. From top to bottom, the black (red, green, blue) lines are for the CHARM (MINOS, U70, T2K) experiments (see section 5.3). The cross sections $\sigma_{n}$ are not appreciably different.

We convert to a per-nucleus cross section by assuming a simple incoherent scaling, $\sigma_{N} \approx$ $Z \sigma_{p}+(A-Z) \sigma_{n}$.

Though we have named this the high-mass regime, we are concerned with particle masses of one to a few GeV. This sets the energy scale for the partonic cross sections, and it is still close to $\Lambda_{Q C D}$. One might then be concerned about possible higher-order corrections in $\alpha_{S}$. We computed these terms, and show show the tree and one-loop cross sections for on- and off-shell processes in figure 14. These plots assume a factorisation scale $\mu_{F}=m_{V}$ (on-shell) or $\sqrt{\hat{s}}$ (off-shell); varying this represents an additional theoretical uncertainty. This can be as large as an $\mathcal{O}(1)$ effect on the overall production rate [87]. We use the NLO cross sections in computing all limits, and note that our limits are selfconsistently comparable where the low- and high-mass regimes meet.

As with hadron production, we obtain the total number of hidden states produced by assuming all interactions take place in the first interaction length with full beam energy. The equivalent of eqs. (4.16) and (5.4) is

$$
N_{X} \approx \sigma_{N} \frac{N_{0} X^{\mathrm{nuc}}}{A} N_{p} .
$$

\subsection{Acceptances}

As for electron beam dump experiments, the experimental acceptance is a product of three terms: an angular acceptance factor that the (meta-)stable particle hits the detector; a survival probability; and the probability of either decaying or scattering within the detector, as appropriate. We operate with samples of hidden sector events rather than the kinematic distributions directly.

In the low mass region, we first construct a set of hadron events, then decay them through the hidden sector spectrum using the narrow width approximation. Our samples are built on uniform grids in the parent hadron phase space, then weighted by the hadronic kinematic distributions. Our choice of weighting function differs by experiment. For LSND and MiniBooNE, we use parameterisations defined in the lab frame as functions of the meson momentum $p_{\text {lab }}$ and $\theta_{\text {lab. }}$ For LSND we use the Burman and Smith fit $f_{B S}$ [92], 
appropriately weighted to account for the use of different target materials over the experiment's lifetime. For MiniBooNE, we use the Sanford-Wang distribution $f_{S W}$ [93]. For all other experiments, we use the modified Bourquin-Gaillard parameterisation $f_{B G}$ [94-96]. This is defined in terms of the CoM frame rapidity $y_{\text {com }}$ and transverse momentum $p_{T, \text { com }}$, so after constructing our initial grid we must boost to the lab frame. Lastly, we decay each parent hadron to the hidden sector multiple times, so as to avoid a rare decay at a high-weight point unduly affecting our results.

In the high mass region, we use an unweighted sample of events construced with standard tools. We implemented our model in FeynRules 2.0 [97-99], and generate events using MadGraph 5.1.5.13 [100] for the processes of figure 13. Since we can produce any hidden sector particle directly in a $2 \rightarrow 2$ process, we do not consider secondary production through the decay of heavier hidden sector particles. This should be a small correction due both to suppressed production of heavier states, and the secondaries having larger production angles relative to the beam axis.

Once we have generated a sample of hidden sector events, the remaining analysis is analogous to section 4.3 . For visible searches, we need modify the decay probability $P_{\text {dec }}$ of eq. (4.20) by replacing $L_{\text {dec }}$ with $L_{d}$. The angular acceptance is also simplified, as we only need the metastable state to cross the detector. In the high mass region, the number of signal events is given by

$$
N_{\text {sig }}=N \times \frac{1}{E V} \sum_{i \in H I T} \mathrm{Br}_{\mathrm{tot}}^{i} \times P_{\mathrm{dec}}^{i},
$$

with $E V$ the number of generated events and HIT the subset of hidden sector particles that hit the detector, with daughter particles that pass the experimental cuts. In the low mass region, the expression is slightly modified:

$$
N_{\text {sig }}=N \times \frac{1}{w_{\mathrm{tot}}} \sum_{i \in H I T} w^{i} \times \mathrm{Br}_{\mathrm{tot}}^{i} \times P_{\mathrm{dec}}^{i},
$$

with $w^{i}$ the weighting associated with the event containing the particle $i$ and $w_{\text {tot }}$ the total weighting summed over all events. The weights in turn are given by

$$
w^{i}= \begin{cases}f_{B S}\left(p_{\text {lab }}^{i}, \theta_{\text {lab }}^{i}\right) \delta p_{\text {lab }} \delta \theta_{\text {lab }} & \text { LSND } \\ f_{S W}\left(p_{\text {lab }}^{i}, \theta_{\text {lab }}^{i}\right) \delta p_{\text {lab }} \delta \theta_{\text {lab }} & \text { MiniBooNE } \\ f_{G W}\left(y_{\text {com }}^{i}, p_{T, \text { com }}^{i}\right) \delta y_{\text {com }} \delta p_{T, \text { com }} & \text { Other experiments }\end{cases}
$$

with $p_{\text {lab }}^{i}$ etc. the parent hadron kinematics, and $\delta p_{\text {lab }}$ etc. the sampling interval in phase space.

For searches for dark matter scattering in the detector, the analysis here is almost identical to to the electron beam dump case. There only modification of note refers to the LSND experiment, which searched for electron scattering with the final state electron in a given energy range. This demands that we use the full scattering cross sections of appendix C, with eq. (4.23) a poor approximation. It is also useful to rewrite the scattering probability as

$$
P_{\text {scat }}=n_{e, N} L_{d} \sigma_{\chi e, N}
$$




\begin{tabular}{|ccrrrrr|}
\hline Experiment & Target & $E_{p}$ & $N_{p}$ & $L_{\text {sh }}$ & $L_{d}$ & $A_{\text {acc }}$ \\
\hline CHARM [101, 102] & $\mathrm{Cu}$ & 400 & $2.4 \times 10^{18}$ & 480 & 35 & 4.8 \\
MINOS [103, 104] & $\mathrm{C}$ & 120 & $1.407 \times 10^{21}$ & 1040 & 1.3 & 3.1 \\
$\nu$-Cal I [105, 106] & $\mathrm{Fe}$ & 70 & $1.71 \times 10^{18}$ & 64 & 23 & 6.76 \\
INGRID [107] & $\mathrm{C}$ & 30 & $5 \times 10^{21}$ & 280 & 0.585 & 21.5 \\
LSND [108, 109] & See text & 0.798 & See text & 30 & 8.3 & 25.5 \\
\hline
\end{tabular}

Table 3. Experimental parameters describing the beam and detector position and size. Energies $E_{p}$ are in $\mathrm{GeV}$, lengths $L_{\mathrm{sh}}$ and $L_{d}$ are in $\mathrm{m}$, and the area $A_{\mathrm{acc}}$ is in $\mathrm{m}^{2} . N_{p}$ is the number of protons on target recorded or expected.

\begin{tabular}{|ccccccc|}
\hline Experiment & $\sigma_{\pi}$ & $\sigma_{\eta} / \sigma_{\pi}$ & $\sigma_{\eta^{\prime}} / \sigma_{\pi}$ & $\sigma_{\rho} / \sigma_{\pi}$ & $\sigma_{\omega} / \sigma_{\pi}$ & $\sigma_{\Delta} / \sigma_{\pi}$ \\
\hline CHARM & See Text & 0.078 & 0.024 & 0.11 & 0.11 & 0.03 \\
MINOS & $229.3 \mathrm{mb}$ & 0.035 & 0.0035 & 0.047 & 0.048 & 0.013 \\
$\nu$-Cal I & $677 \mathrm{mb}$ & 0.035 & 0.0035 & 0.049 & 0.049 & 0.014 \\
INGRID & $229.3 \mathrm{mb}$ & 0.035 & 0.0035 & 0.046 & 0.047 & 0.017 \\
\hline
\end{tabular}

Table 4. Hadron cross sections we take for low mass hidden sectors. Except as mentioned in the text, we take $\sigma_{\pi} \approx 25-30 \sigma_{\eta}$ from ref. [110, 111], and the multiplicities of other hadrons from Pythia 8.180 [112, 113].

With this correction, the total signal yield in the high mass region is given by eq. (4.26). In the low-mass region, we modify it to include the weighting,

$$
N_{\text {sig }}=N \times \frac{1}{w_{\text {tot }}} \sum_{i \in H I T} w^{i} \times \mathrm{Br}_{\text {tot }}^{i} \times P_{\text {scat }}^{i} \times P_{\text {surv }}^{i},
$$

with $w^{i}$ as given in eq. (5.11).

\subsection{Experiments}

We list general properties of the experiments we find set non-trivial limits in table 3 , and hadron production cross sections in table 4. Properties relevant to visible searches are in table 5, and to invisible scattering searches in table 6 . Because there is a relatively large variation in the configuration and searches for each experiment, we discuss them individually below.

CHARM. The CHARM experiment featured a $400 \mathrm{GeV}$ proton beam impacting a copper target. Searches where performed for an axion (pseudoscalar) decaying to $\gamma \gamma, e^{+} e^{-}$or $\mu^{+} \mu^{-}$in ref. [101]; and for heavy neutrinos decaying as $\nu_{h} \rightarrow \nu l^{+} l^{-}$in ref. [102]. These cover the relevant topologies of two- and three-body decays with and without additional unobserved states. No events where observed in either search.

The CHARM detector was positioned $5 \mathrm{~m}(10 \mathrm{mrad})$ off the beam axis and had a square transverse cross section. For low mass hidden resonances, we normalise our results to $N_{\pi}^{\text {det }}=2.9 \times 10^{17}$ from the experimental collaboration [101], where $N_{\pi}^{\text {det }}$ is the number of pions produced on a trajectory that would intersect the detector. For other pseudoscalar mesons, we use the cross sections quoted in ref. [86]; for vectors, the mea- 


\begin{tabular}{|crrrrr|}
\hline Experiment & $E_{\mathrm{thr}}^{e}$ & $E_{\mathrm{thr}}^{\mu}$ & $\kappa_{\mathrm{Eff}}^{e}$ & $\kappa_{\mathrm{Eff}}^{\mu}$ & $N_{u p}$ \\
\hline CHARM & 5 & 5 & 0.51 & 0.85 & 3 \\
MINOS & - & 1 & - & 0.8 & 10 \\
$\nu$-Cal I & 3 & 3 & 0.7 & 0.9 & 7.76 \\
LSND & 0.015 & - & 0.19 & - & $10^{3}$ \\
\hline
\end{tabular}

Table 5. Detector parameters relevant for visible searches. Energies $E_{\mathrm{thr}}^{e}$ and $E_{\mathrm{thr}}^{\mu}$ are in $\mathrm{GeV}$, while $\kappa$ are detection efficiencies. Dashes signify a column does not apply for that experiment. See table 3 or the text for references.

\begin{tabular}{|ccccc|}
\hline Experiment & $n_{e}$ & $n_{N}$ & $\kappa_{\text {eff }}$ & $N_{u p}$ \\
\hline MINOS & - & $5 \times 10^{24}$ & 0.8 & $10^{3}-10^{4}$ \\
INGRID & - & $5 \times 10^{24}$ & 0.8 & $10^{3}-10^{4}$ \\
LSND & $5.1 \times 10^{23}$ & - & 0.19 & $10^{3}$ \\
\hline
\end{tabular}

Table 6. Detector parameters for invisible searches. Dashes signify a column is not relevant for that experiment. See table 3 or the text for references.

surements of refs. $[114,115]$; and for fermions, we estimate $N_{\Delta} \approx 0.03 N_{\pi}$ from the relative multiplicities in Pythia 8.180 [112, 113].

Limits from these CHARM searches have previously been interpreted in terms of vector kinetic mixing. Ref. [86] only considered the decay $Z^{x} \rightarrow l^{+} l^{-}$, with the hidden vector produced in psuedoscalar meson decay. Ref. [25] did consider production and decay of hidden scalars, but in a non-supersymmetric context and at high masses. Our results are then different due to the mass mixing, as discussed in appendix A.

MINOS. The MINOS experiment [103] uses the $120 \mathrm{GeV}$ NuMI beam on a graphite target. We consider possible signals in the near detector, positioned $1.04 \mathrm{~km}$ away. We model the detector as a cylinder of radius $1 \mathrm{~m}$ and take a recent value [104] for the total number of protons on target. For hadron production we take the cross section for $p C \rightarrow$ $\pi+\ldots$ from ref. [116]; using eq. (5.4) gives $N_{\pi} \approx 0.99 N_{p}$.

We consider the possibility of signals at MINOS from both hidden sector scattering and decay. For decays, we follow ref. [17] and consider a sensitivity to $\mathcal{O}(10)$ muon pairs with a detection efficiency comparable to that for scattering [117]. Searches for invisible particle scattering must compete against a large neutrino background. We follow ref. [87] and show prospective limits for $10^{3}$ and $10^{4}$ events in our plots, corresponding to different estimates for background rejection.

$\boldsymbol{\nu}$-Cal I. The $\nu$ Calorimeter I experiment took data from a beam dump at the U70 accelerator, where a $70 \mathrm{GeV}$ proton beam was delivered to an iron target. Searches for axions and light Higgs bosons [105, 106] were reinterpreted in terms of hidden vectors decaying to leptons in refs. $[88,89]$. We find the pion production cross section by rescaling 
the measurement of ref. $[118,119]$ as discussed in [89]:

$$
\sigma(p F e \rightarrow \pi+\ldots) \approx A^{0.55} \times \sigma(p p \rightarrow \pi+\ldots) .
$$

From eq. (5.4) we find $N_{\pi} \approx 0.96 N_{p}$. Also, our choice for the $\eta$ production cross section is consistent with its non-observation in ref. [118, 119].

The combination of a relatively small distance between the target and the detector and a moderately large energy are useful in setting limits when the mass mixing of appendix A dominates the $h_{1}^{x}$ decay. However, we find that the limits from meson decays are almost always inferior to the corresponding limits from CHARM.

INGRID. INGRID [107] is the near on-axis detector in the T2K experiment [120]. We use an estimate for the prospective total lifetime $N_{p}$, and approximate the detector efficiencies as similar to MINOS. The detector has a non-trivial cross shape transverse to the beam direction. As with MINOS, a large neutrino background would demand a dedicated study to reject backgrounds, which we approximate by considering limits for $10^{3}$ and $10^{4}$ events.

LSND. The LSND experiment used a $800 \mathrm{MeV}$ proton beam on two different targets over its lifetime, water and a high-Z metal [108]. When computing the acceptance efficiencies, we use a weighted mean of the two distribution functions $f_{B S}$ for the different target materials. We model the detector as a cylinder of diameter $5.7 \mathrm{~m}$. Because of the low beam energy, we only consider limits from hidden states produced in pion decays; it is assumed that the production of heavier hadrons is highly kinematically suppressed. We use an estimate $N_{\pi} \approx 10^{22}$ of the number of pions produced from [21].

A search for neutral current scattering on the full LSND data set observed $\mathcal{O}(300)$ events above background, of which $\mathcal{O}(200)$ where expected from neutrinos [109]. We conservatively place limits for 1000 hidden sector events as unambiguously ruled out [20, 21]. Along with MINOS, we consider the possibility of both hidden sector decay (to electrons) and scattering, as shown in tables 5 and 6 , though we find that the limits from visible decays are only as good as those from CHARM.

Other experiments. Searches for heavy neutrinos decaying to leptons were carried out at NOMAD [121] and PS-191 [122, 123], and reinterpreted as searches for hidden vectors in ref. [90]. We find that the limits from other hidden decays then $Z^{x} \rightarrow l^{+} l^{-}$are inferior to those from CHARM. Refs. [20, 21, 87] proposed looking for hidden particles scattering at MiniBooNE [124] and in the ND280 detector at T2K [125]. For our benchmarks, the former is always inferior to MINOS and the latter to INGRID. Finally, we note that future experiments such as Project X [126, 127] or AFTER@LHC [128, 129] would likely place further exclusions on our paramter space. The nature of those limits is beyond the scope of this work.

\subsection{Limits}

We now present current and prospective limits for our four benchmarks from hadronic beam dumps. Composite plots showing all the limits we find are shown in section 7 . We give limits for Cases A, B and D in figures 15, 16 and 19. For Case C, we show 
limits separately for constraints from scalars (figure 17) and pseudoscalars (figure 18) for clarity. In figures 15, 16 and 17, we split the constraints for $m_{x}>0.1 \mathrm{GeV}$ with (left) and without (right) the inclusion of D-term Higgs portal mass mixing. As discussed in appendix A, the presence of this mass mixing heavily alters the $h_{1}^{x}$ lifetime above the muon threshold. Hence all experimental searches based on a long-lived scalar will differ between these two possibilities.

We note some general features of our results before discussing each case in detail. Limits from the hidden Higgs decays are almost always upper bounds on $\epsilon$. This is because for kinetic mixings not ruled out by e.g. EWPTs, the scalar decay length is large. Signals are then limited by production $\left(\propto \epsilon^{2}\right)$ and, when relevant, scattering probability $\left(\propto \epsilon^{2}\right)$. The exception comes only for large masses $m_{h_{1}^{x}}>2 m_{\mu}$, when the lifetime can become short enough for the scalar to decay within the shield.

At low masses, limits from LSND tend to dominate due to its large luminosity and the absence of kinematic suppression. At intermediate masses, limits from CHARM are stronger; and at high masses above the muon threshold, the $\nu$-cal I search can also be relevant. We show current limits derived here in shaded regions, and use unshaded contours to denote prior exclusions or new prospective limits. For MINOS, we label possible limits from visible searches as "MINOS(VIS)". For both MINOS and INGRID, we use " $10^{3}$ " and " $10^{4}$ " for contours of that many expected scattering events. We omit any results whose exclusions would be entirely within those of another experiment; this means that not all experiments appear in all plots.

Case A. In this benchmark the hidden vector will decay to the SM, so we include limits for this from NOMAD and PS-191 [86], $\nu$-calorimeter I [88, 89], and CHARM [90]. These extend to lower values of the kinetic mixing than the channels we consider here, but the exclusions cannot reach large values of $\epsilon$ as the $Z^{x}$ lifetime becomes too short.

We find three new limits, shown as shaded regions in figure 15. The first is a limit at LSND from $h_{1}^{x}$ scattering; it arises at low masses where the $h_{1}^{x}$ is stable over the relevant length scales. Additionally we have limits at CHARM and $\nu$-Cal I from $h_{1}^{x}$ decays. We split the CHARM limits into two regions. The darker region with dashed boundary shows the limits when the hidden sector is produced through the decay of a pseudoscalar meson, or through $q \bar{q}$ fusion at high masses. The light region with dotted boundary shows limits from the decay $\rho \rightarrow Z^{x} h_{1}^{x}$. The resonance at $m_{x} \approx m_{\rho}$ is clearly visible. These limits are stronger as they arise from a two-body decay, which compensates for the smaller $\rho$ multiplicity. However, there is a larger systematic uncertainty in the number of $\rho$ produced. The shown limits from $\nu$-Cal I are for production in $q \bar{q}$ fusion only; the limits at lower masses are inferior to the corresponing CHARM constraints.

The limits from INGRID, and from MINOS due to dark particle scattering, also come from the $\rho$. While the decay $\rho \rightarrow \chi_{1} \chi_{1}$ is possible, the large fermion mass means our limits are set by $\rho \rightarrow Z^{x} h_{1}^{x}$, with the $h_{1}^{x}$ effectively stable in this mass range.

At high masses, we can clearly see the effect of the Higgs mass mixing by comparing the left (with) and right (without) sides of figure 15. With the mass mixing, the limits from $q \bar{q}$ fusion at $\nu$-Cal I are strictly superior to those from CHARM, and we do not show 

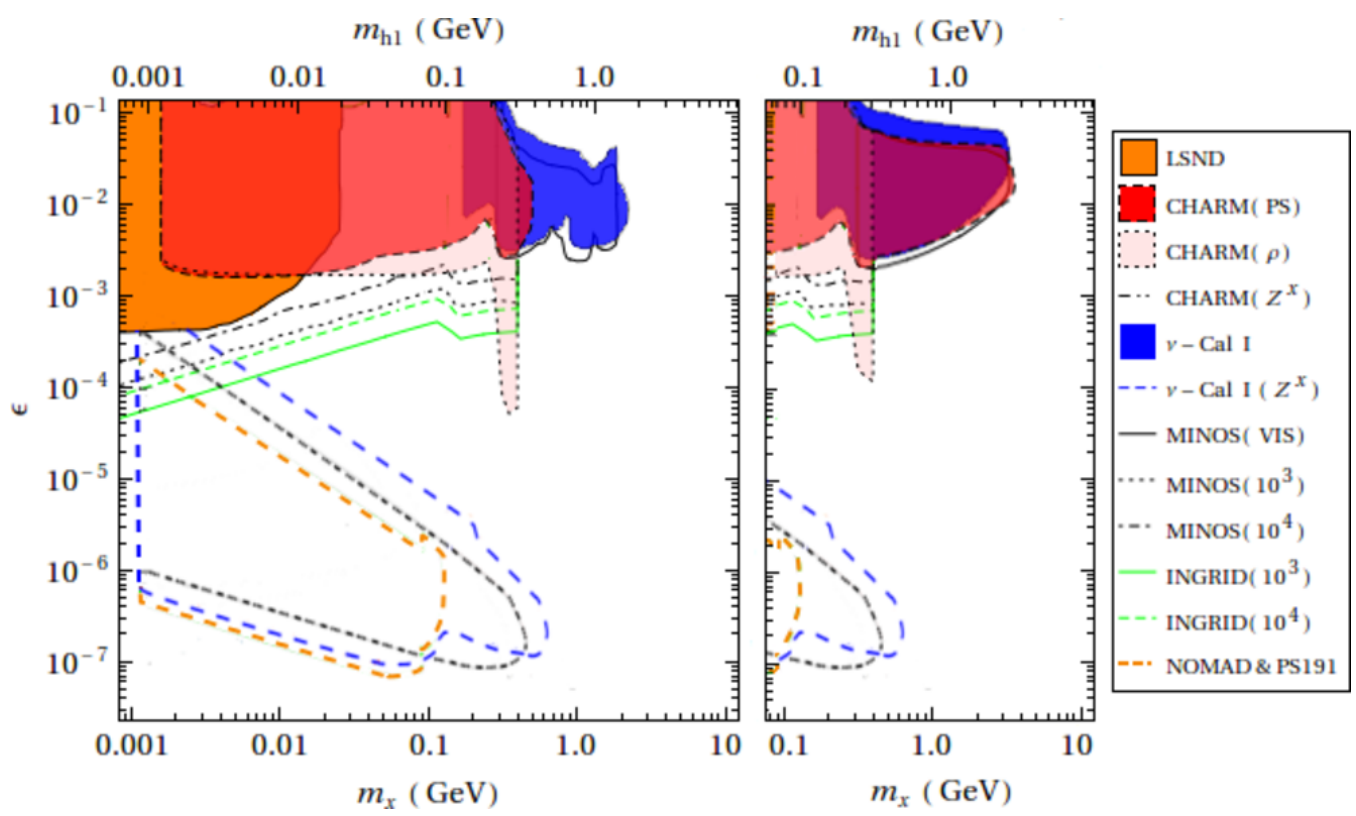

Figure 15. Case A, with (left) and without (right) the Higgs mass mixing induced by the SUSY D-terms. The unshaded dashed contours show prioir limits from $Z^{x}$ decays for CHARM (black), $\nu$ Cal I (blue), and NOMAD and PS-191 (orange). Shaded regions show new limits: from $h_{1}^{x}$ decays at CHARM (red and pink) and $\nu$-Cal I (blue), and from $h_{1}^{x}$ scattering at LSND (orange). The solid black contour shows prospective limits from $h_{1}^{x}$ decays at MINOS. The remaining contours show prospective limits from $h_{1}^{x}$ scattering at MINOS (black dotted or dot-dashed) and INGRID (green solid or dashed), with different contours for sensitivity to $10^{3}$ or $10^{4}$ events. See the text for more details.

the latter. We also have potential limits from decays to muons at MINOS. The unusual shape of these limits is due to a resonance in the Higgs decay near the $K^{0}$ mass. Without the mass mixing, we can exclude higher masses and the higher centre of mass energy of CHARM becomes important.

Case B. For visible searches, this benchmark point differs from Case A in two regards. The main difference is that there are no longer any limits from $Z^{x} \rightarrow l^{+} l^{-}$, as previously discussed. For limits from other hidden particle decays, apart from small differences in the ratio $m_{h_{1}^{x}} / m_{x}$ and the mixing matrix $R$, the two sectors are effectively the same. It follows that the associated limits are nearly identical. These are the limits from CHARM and $\nu$-Cal I, and the visible decay prospects from MINOS, shown in figure 16.

However, the possibility for the hidden vector to decay to two hidden fermions, $Z^{x} \rightarrow$ $\chi_{1}^{x} \chi_{1}^{x}$, substantially enhances the limits from dark sector scattering. We can easily see this by comparing the LSND, MINOS and INGRID exclusions and prospects between figures 15 and 16. At low masses, hidden states at MINOS and INGRID limits are dominantly produced from pions, as this can now produce hidden sectors through successive two-body decays. Above the pion mass the contributions from the $\rho$ and $\eta$ are comparable, and at high masses quark fusion is the dominant channel. Finally, we note that the limits here are not directly comparable to those shown in e.g. refs. [1, 24]. Those limits assumed a fixed $\chi_{1}^{x}$ mass and varied the vector mass, while we fix the mass ratio and vary them together. 


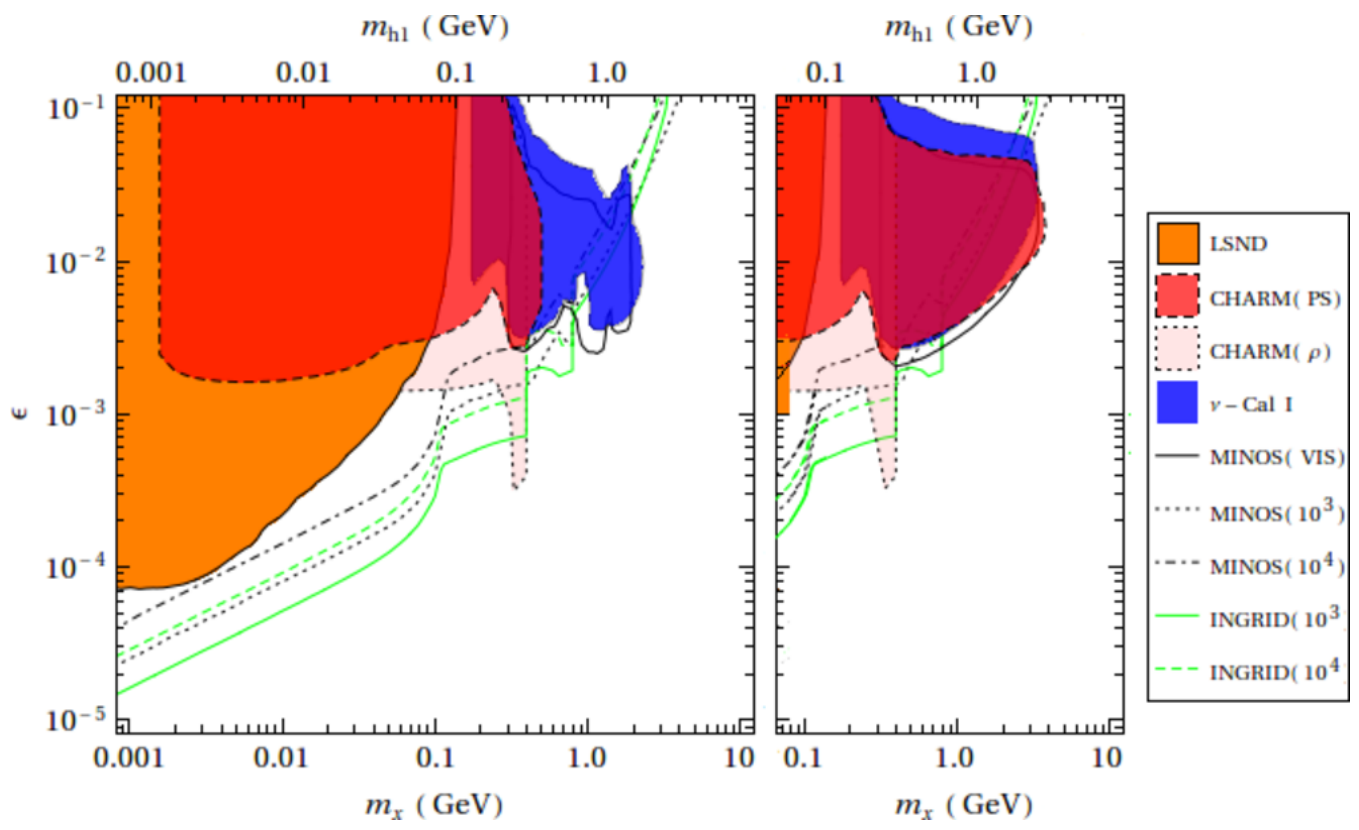

Figure 16. Case B, with (left) and without (right) the Higgs mass mixing. Shaded regions show new limits: from $h_{1}^{x}$ decays at CHARM (red and pink) and $\nu$-Cal I (blue), and from $\chi_{1}^{x}$ scattering at LSND. The solid black contour shows prospective limits from $h_{1}^{x}$ decays at MINOS. The remaining contours show prospective limits from $\chi_{1}^{x}$ scattering at MINOS (black dotted or dot-dashed) and INGRID (green solid or dashed), with different contours for sensitivity to $10^{3}$ or $10^{4}$ events. See the text for more details.
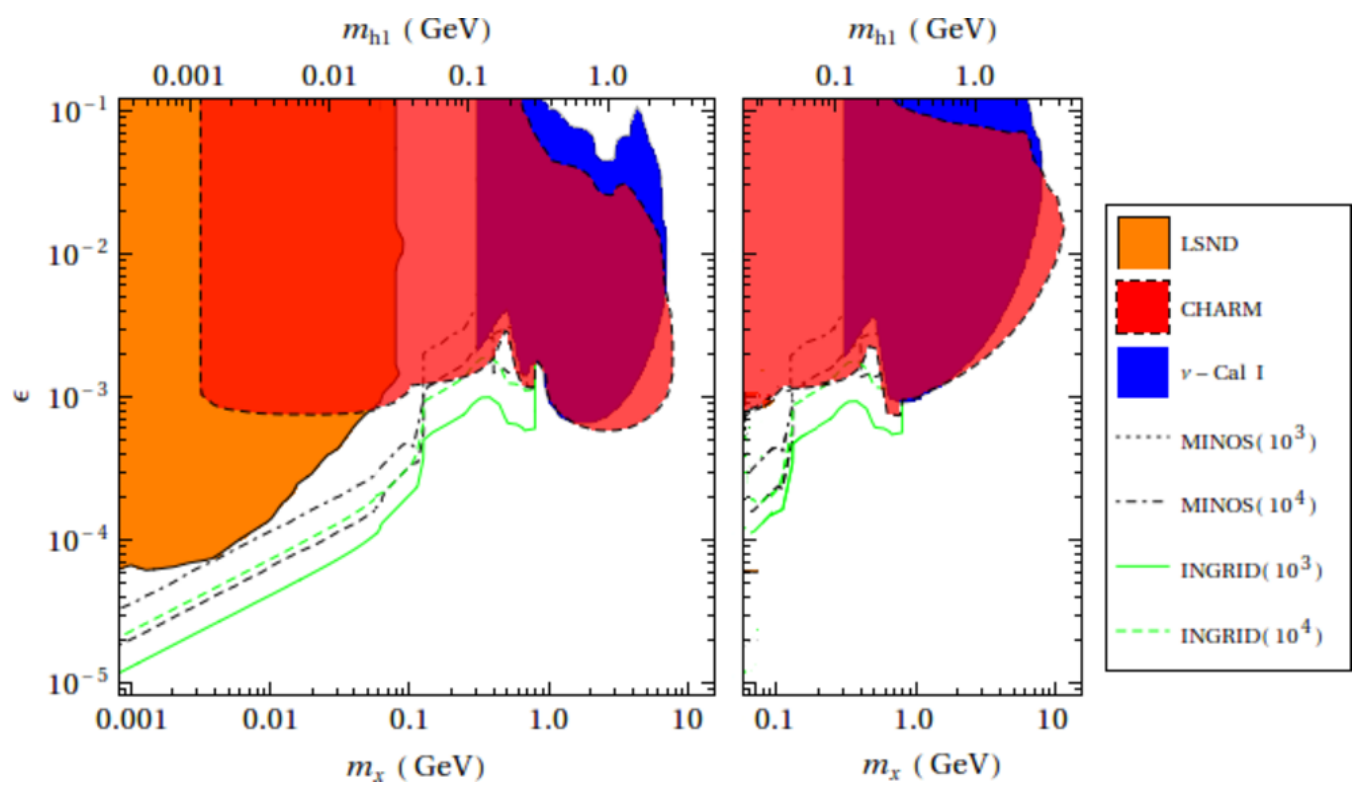

Figure 17. Case C, with (left) and without (right) the Higgs mass mixing. We show here only limits from hidden sector scattering and from $h_{1}^{x}$ decays. The decay limits at CHARM and $\nu$-Cal I have the same notation as in figures 15 and 16. The limits at LSND, and prospective limits at MINOS and INGRID, receive roughly equal contributions from $h_{1}^{x}$ and $A^{x}$ scattering. 


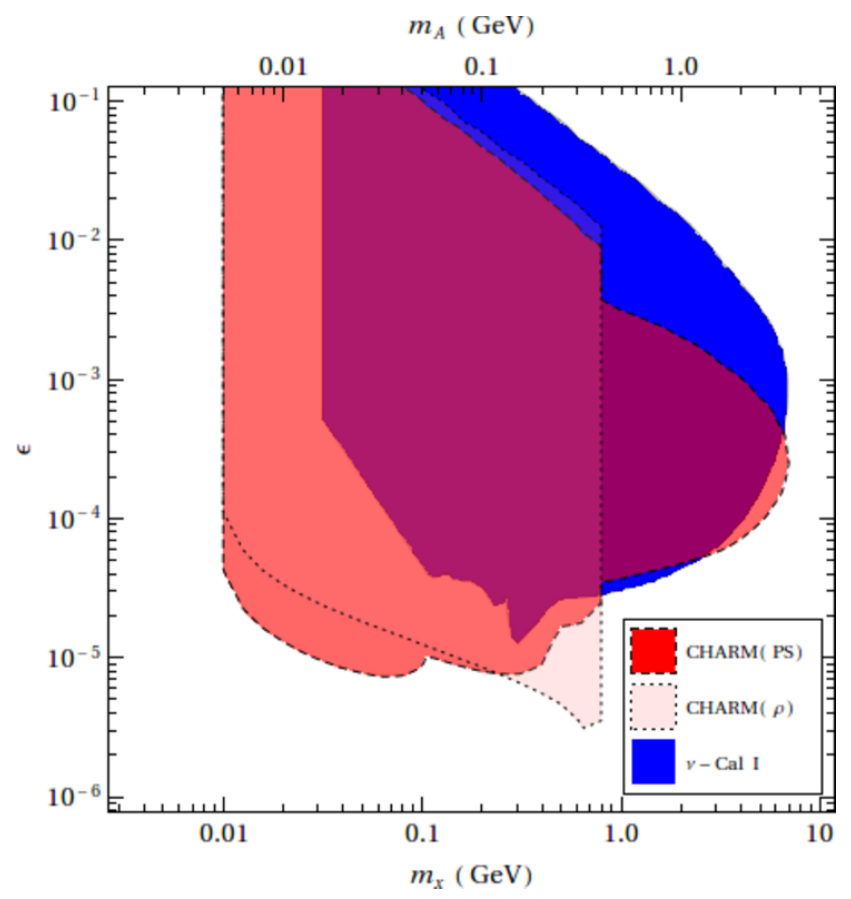

Figure 18. Case C, limits from pseudoscalar decays $A^{x} \rightarrow h_{1}^{x} e^{+} e^{-}$only. The limits from $\nu$-Cal I at low masses and mixings are worse than the CHARM limits, but beyond that are only approximate.

Case C. The ability to produce the scalars through an on-shell vector substantially enhances the limits we derive. Additionally, the $A^{x}$ can be long-lived and is also produced in the on-shell decay of a hidden vector, so we also find limits from its decays. To avoid our plots becoming too cluttered to read, and because they are independent of the Higgs mass mixing, we separate the limits from $A^{x}$ decays into figure 18.

In figure 17 we show all other limits in this benchmark. In particular, the exclusions from $h_{1}^{x}$ decays at both CHARM and $\nu$-Cal I extend to higher masses and lower mixings than in cases A and B. We note that the limits we find at CHARM without the mass mixing and at high masses are comparable to those derived in ref. [25]. The limits at low masses at CHARM are dominated by pseudoscalar decays, which can now produce hidden states through two-body channels, e.g. $\pi^{0} \rightarrow \gamma Z^{x}$ followed by $Z^{x} \rightarrow h_{1}^{x} A^{x}$.

The limits from scattering experiments come from both $h_{1}^{x}$ and $A^{x}$, which give roughly equal contributions. This is to be expected from eqs. (4.23) and (4.25). Hidden states are again dominantly produced by pions when allowed, and from $\eta$ and $\rho$ decays when not.

The limits from the pseudoscalar in figure 18 all come from the visible decay $A^{x} \rightarrow$ $h_{1}^{x} e^{+} e^{-}$. They are interesting in that the exclusion contours have relevant upper bounds. The $A^{x}$ decay is less suppressed than the hidden Higgs, and so it can be sufficiently shortlived to decay before reaching the detector. This will happen at large mass and kinetic mixing. The $\nu$-Cal I experiment sets better limits in this domain for the simple reason that the detector was closer to the interaction point. At low masses, the limits from CHARM dominate, with the $\rho$ resonance visible. 


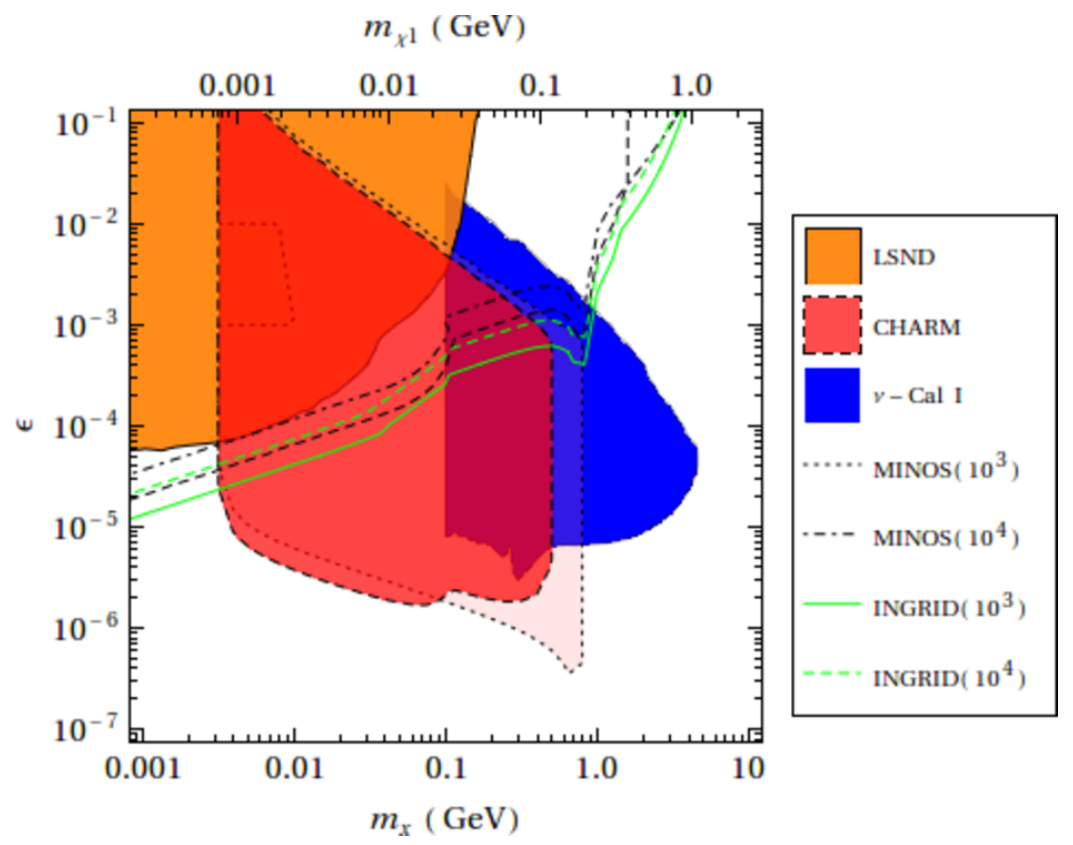

Figure 19. Limits in Case D. Limits from $\chi_{x}^{2} \rightarrow \chi_{x}^{1} e^{+} e^{-}$or $\chi_{1}^{x} \mu^{+} \mu^{-}$are shown in red or pink (CHARM) and blue ( $\nu$-Cal I). Limits from $\chi_{1,2}^{x}$ scattering from LSND are shown in orange. Prospective scattering limits from MINOS and INGRID are shown as black and green contours, respectively.

Case D. Unlike the three previous cases, there are no limits in this benchmark from metastable scalars. All limits come from the production of hidden sector fermions. We have significant limits both from $\chi_{2}^{x}$ decays to leptons, and from $\chi_{1}^{x}$ scattering $\left(\chi_{2}^{x}\right.$ scattering is a subleading effect). Finally, because we have no limits from $h_{1}^{x}$ we have no sensitivity to the Higgs mass mixing, and hence show only a single plot in figure 19.

The constraints from visible searches are dominated by CHARM at low mass and $\nu$-Cal I at high mass. Hidden fermion production at CHARM is dominated by pion decay when kinematically accessible, and by $\rho$ decay when not. The $\rho$ wins out over the $\eta$ mostly due to higher angular acceptances. Hidden fermion production from pseudoscalars requires two two-body decays, and so they tend to have a larger angle relative to the beam axis. Like the pseudoscalar in Case C, the fermion decays are sufficiently less suppressed than the $h_{1}^{x}$ for our exclusion contours to have an upper bound. This occurs when the $\chi_{2}^{x}$ lifetime is so short, it decays before reaching the detector. The superiority of the $\nu$-Cal I experiment at high masses is due to its location closer to the detector.

Limits from scattering experiments are straightforward. Of particular note is their sensitivity at high mass, where the $\chi_{2}^{x}$ is decays too promptly. In this region the MINOS and INGRID limits can be very important. Otherwise, limits from visible searches win so long as we are above the electron threshold.

\section{Implications at the LHC}

Signatures of this theory at the LHC were investigated in ref. [26]. When the MSSM superpartners are near the $\mathrm{TeV}$ scale, hidden states can be produced readily in supersymmetric 
cascades refs. [9, 130-132]. In general, the creation of a pair of MSSM superpartners will initiate a pair of visible cascades down to the lightest SM superpartner (LSMP). Each LSMP will subsequently decay to the hidden sector, thereby initiating a hidden cascade. Depending on the decay properties of the hidden states, this may produce additional visible activity in the event and reduced missing energy $\left(B_{T}\right)$. The extent to which this occurs is related to the signatures of the hidden sector in low-energy experiments.

MSSM superpartners connect to the hidden sector through the supersymmetric kinetic mixing of eq. (2.2) between the Bino and the $\mathrm{U}(1)_{x}$ gaugino. When the LSMP is a neutralino with a significant Bino component, the decays $\chi_{1}^{0} \rightarrow \chi_{i}^{x} S^{x}$, where $S^{x}=Z^{x}, h_{1}^{x}, h_{2}^{x}, A^{x}$, are likely to be prompt [131]. As in ref. [26], we will focus on this case here. Other species of LSMPs tend to have delayed decays, possibly leading to charged tracks [131]. The decay of an LSMP neutralino populates the four bosonic states equally, while the relative fractions of the hidden neutralino species depends on their hidden Higgsino contents [26].

Decays of the $\chi_{1}^{0}$ LSMP will contribute to missing energy, but they can also produce visible signals. The latter occurs when a vector, on- or off-shell, is created in the cascade, and decays to SM states. In contrast, direct decays of $h_{1}^{x}$ to the SM are usually too slow to occur within the LHC detector. Since we assume $m_{\chi_{1}^{0}} \gg m_{x}$, the boson and the fermion produced by a $\chi_{1}^{0}$ decay will be highly boosted by an amount $\gamma \sim m_{\chi_{1}^{0}} / 2 m_{x}$. Any visible products created along the subsequent boson and fermion decay chains will therefore be highly boosted and collimated. Following ref. [26], we will call these hidden-valley (HV) jets. An HV jet may contain leptons or hadrons, and it can be prompt or displaced. Each $\chi_{1}^{0}$ decay can produce zero, one, or two HV jets.

The average number of HV jets per $\chi_{1}^{0}$ decay depends on the spectrum of the hidden sector, and is related to the signals expected in low-energy experiments. In case A, the HV jets come mostly from on-shell vectors and can be prompt or delayed, with

$$
\gamma c \tau \sim 500 \mu \mathrm{m}\left(\frac{10^{-3}}{\epsilon}\right)^{2}\left(\frac{0.1 \mathrm{GeV}}{m_{x}}\right)^{2}\left(\frac{m_{\chi_{1}^{0}}}{100 \mathrm{GeV}}\right)
$$

Hidden vectors are produced directly from the Bino decay, or in a two-step decay $\chi_{1}^{0} \rightarrow$ $A^{x} \rightarrow Z^{x}$. The heavier scalar preferentially decays $h_{2}^{x} \rightarrow h_{1}^{x} h_{1}^{x}$. At least one HV jet will be present in $3 / 4$ of supersymmetric events.

In case B, nearly all LSMP decays are completely invisible. With the exception of $h_{1}^{x}$, the dominant decay channel of all the bosons in the theory is $S^{x} \rightarrow \chi_{1}^{x} \chi_{1}^{x}$. While the $h_{1}^{x}$ state does decay to the SM, it is typically too slow to be seen in the LHC detectors.

Case $\mathrm{C}$ can yield $\mathrm{HV}$ jets when $A^{x}$ pseudoscalars are created in cascades. These decay through $A^{x} \rightarrow h_{1}^{x}+Z^{x *}$ with $Z^{x *}$ producing an $\mathrm{HV}$ jet. However, these $\mathrm{HV}$ jets are frequently displaced or delayed when the boost from the $\chi_{1}^{0}$ decay is included. Case D is similar to case $\mathrm{C}$, but now the $\mathrm{HV}$ jets come mainly from the vector in $\chi_{2}^{x} \rightarrow \chi_{1}^{+} Z^{x *}$ decays. Again, these are likely to be delayed. This is especially true given that our limits in these benchmarks tend to force the kinetic mixing to be small, $\epsilon \lesssim 10^{-4}-10^{-3}$. 

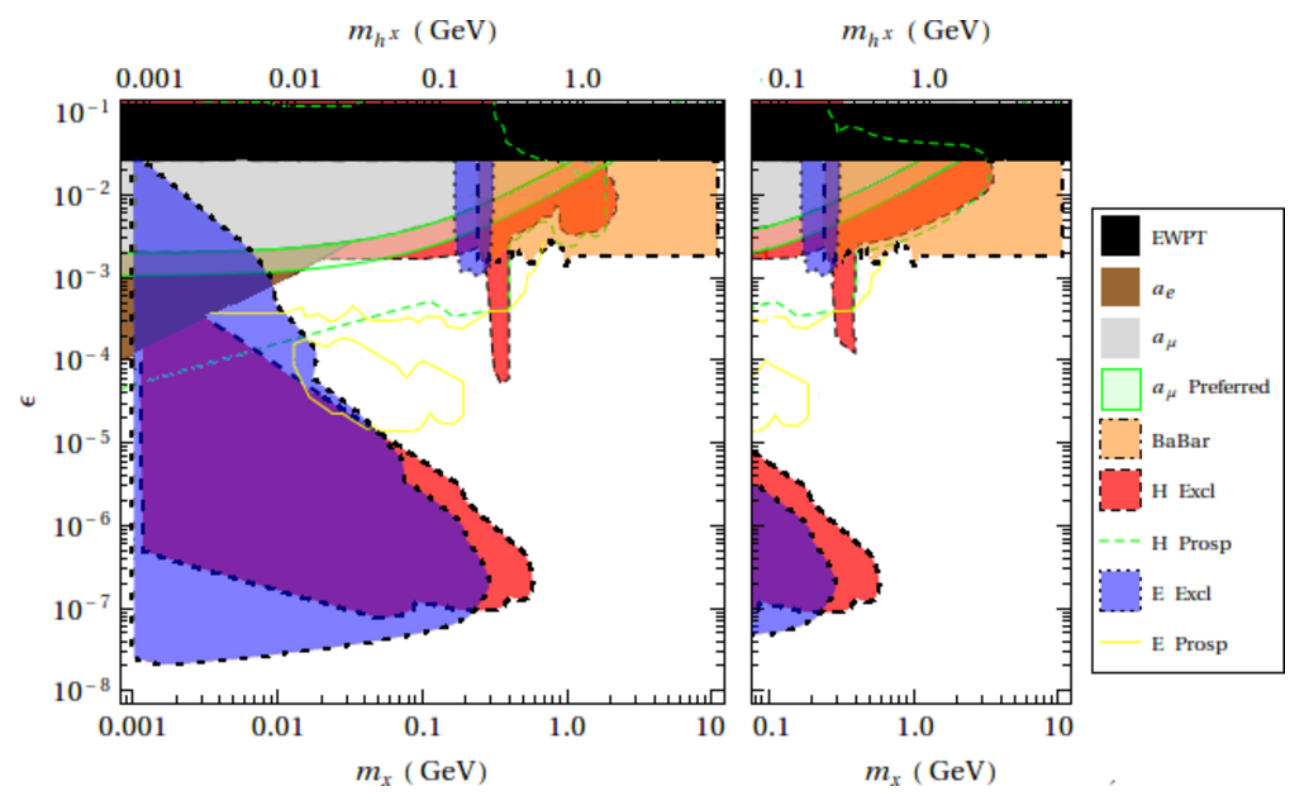

Figure 20. Composite plot of all limits we find for Case A. The exclusions from electron beam dumps, and from proton beam dumps at low $m_{x}$ and $\epsilon$, come from the decay $Z^{x} \rightarrow l^{+} l^{-}$. These are as cited in sections 4 and 5, plus the exclusions from the MAMI [68] and APEX [69] test runs. We do not include the new electron limits from figure 9, as they are inferior to those from the anomalous magnetic moment. Prospective limits from electron beam dumps are the union of the APEX [71], HPS [73], DarkLight [72], MAMI and MESA [133] projections. The limits from BaBar are from refs. [1, 18, 19, 39]. Not shown are limits from KLOE [41, 42] and WASA-at-COSY [43], which are slightly weaker than the CHARM limits.

\section{Conclusions}

In this work we have investigated the limits on a minimal supersymmetric hidden sector from lower-energy precision experiments. This model provides a well-defined and plausible extension of the simplified hidden-sector theories studied previously. In certain limits, our model behaves nearly identically to some of these simplified models. However, our supersymmetric extension can also lead to richer and more complicated signals that have received little attention so far.

To survey the range experimental signatures of this model, we have focussed on four specific parameter slopes that cover the bulk of the most likely possibilities. For each slope, the dimensionful parameters of the theory are varied in fixed ratios to the vector mass $m_{x}$. In Case $\mathrm{A}$, the vector $Z^{x}$ has no hidden decay channels and goes exclusively to the SM. In Case B, the vector decays almost completely invisibly to the lightest stable hidden neutralino $\chi_{1}^{x}$. In Case $\mathrm{C}$, the vector decays primarily to hidden scalar states which decay in turn to the SM, typically with a significant delay. In Case D, the vector decays to both the $\chi_{1}^{x}$ and $\chi_{2}^{x}$ hidden fermions, with $\chi_{2}^{x}$ subsequently decaying $\chi_{1}^{x}$ and SM states.

Of these, Cases A and B lead to signals that are very similar to the commonlyconsidered scenarios of a hidden vector decaying to the SM (Case A) [19], and invisibly (Case B) $[22,24]$. Cases $\mathrm{C}$ and $\mathrm{D}$ have not been studied in nearly as much detail, and 
we have substantially extended (Case C) or performed the first study of (Case D) the exclusions that apply in these cases. Additionally, we have investigated how the presence of more states in the hidden sector can affect the limits even in Cases A and B. In all four cases, producing hidden sector states through off-shell vectors or through the decay of a vector meson grant access to new detection channels. Of particular importance is the production and decay of the hidden sector Higgs, which sets new limits in phenomenologically interesting regions of parameter space. We have also studied how limits from hidden scalar decays vary depending on whether $D$-term mixing with the visible Higgs (as expected in our supersymmetric realization) is included or not.

Summary plots containing the limits derived on all four scenarios are shown in figures 20-23, together with some additional limits taken from the literature. We do not include the limits from section 3.3 from cosmologically late decays since they depend on the detailed cosmological history. To simplify our plots, we combine all exclusions from electron fixed target experiments into one region, and from proton beam dumps into another. Similarly, we show a single line for prospective limits from current and future experiments, amounting to the most optimistic scenarios we considered: specifically, observation of 40 events at JLab, 1000 events at INGRID and 10 muon pairs at MINOS. For Cases A-C, we show separately the high mass $\left(m_{x}>0.1 \mathrm{GeV}\right)$ region both with (left) and without (right) the Higgs mass mixing.

In Case A, the most important new feature in figure 20 relative to the exclusions for minimal models is the region excluded by CHARM from decays $\rho \rightarrow h_{1}^{x} Z^{x}$. These are the strongest limits for $30 \mathrm{MeV} \lesssim m_{x} \lesssim 150 \mathrm{MeV}$ and $250 \mathrm{MeV} \lesssim m_{x} \lesssim 400 \mathrm{MeV}$. In particular, they exclude almost all of the region of parameter space where the hidden vector can explain the apparent discrepancy in the muon anomalous magnetic moment. We also note that the prospective limits from INGRID are stronger than those from DarkLight/MESA for $m_{x} \lesssim 40 \mathrm{MeV}$.

In Case B (figure 21), new exclusions relative to minimal models are found from CHARM between $60 \mathrm{MeV} \lesssim m_{x} \lesssim 400 \mathrm{MeV}$. They again exclude most of the $a_{\mu}$-preferred region, with the rest excluded by LSND. Unlike in Case A, there is some parameter space sensitive to the Higgs mass mixing that is not excluded by BaBar: a region around $m_{x} \approx 1 \mathrm{GeV}$ can be excluded by MINOS with the mass mixing, but not without it.

The exclusion of the $a_{\mu}$-preferred region can be avoided in generic hidden sectors in two ways: giving the hidden vector a Stückelberg mass, or making the hidden Higgs decay to the hidden sector. If the hidden vector has a Stückelberg mass, then there is no hidden Higgs and the limits obviously do not apply. Higgs decays of the form $h_{1}^{x} \rightarrow \chi^{x} \chi^{x}$ also trivially sidestep these constraints. Note, though, that in Case A this is only possible if $m_{h_{1}^{x}}>m_{x}$, which is not possible in our minimal supersymmetric model. Finally, a more complex hidden sector might force the hidden states produced in the $\rho$ decay to return to the visible sector only after a complex hidden cascade; such a scenario might suppress the total acceptance enough that no limits can be set.

In Case C (figure 22), we have limits that substantially extend the reach and type of those of ref. [25] in the supersymmetric hidden sector we consider. We have divided the excluded regions from fixed target experiments in two. The upper regions can be excluded 

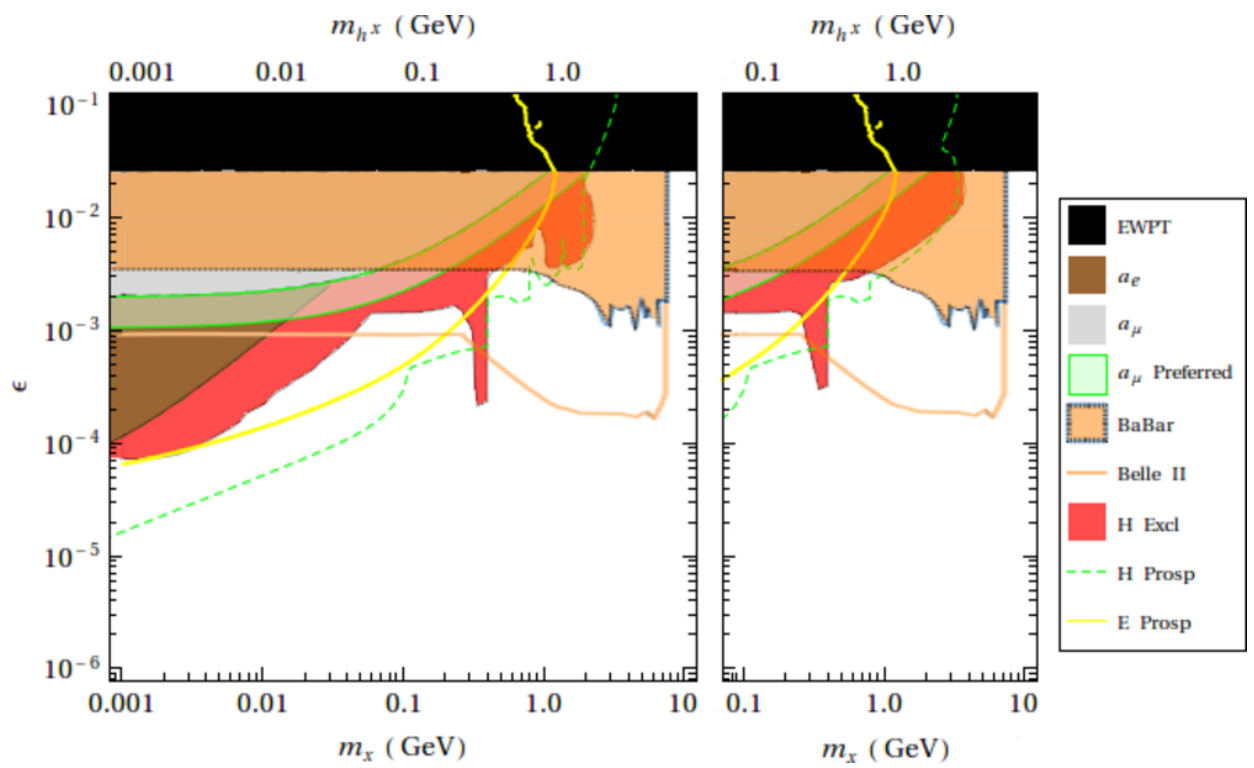

Figure 21. Composite plot of all limits we find for Case B. The limits from BaBar and prospective exclusions from Belle II are estimated from refs. [22, 24, 47] and [24] respectively. Limits from invisible kaon decays $[16,49]$ are comparable to limits from CHARM for $m_{x} \sim 80-100 \mathrm{MeV}$, but otherwise weaker. Prospective limits from ILC are beyond the scope of this work, but are likely slightly better than those from JLab [22, 134].
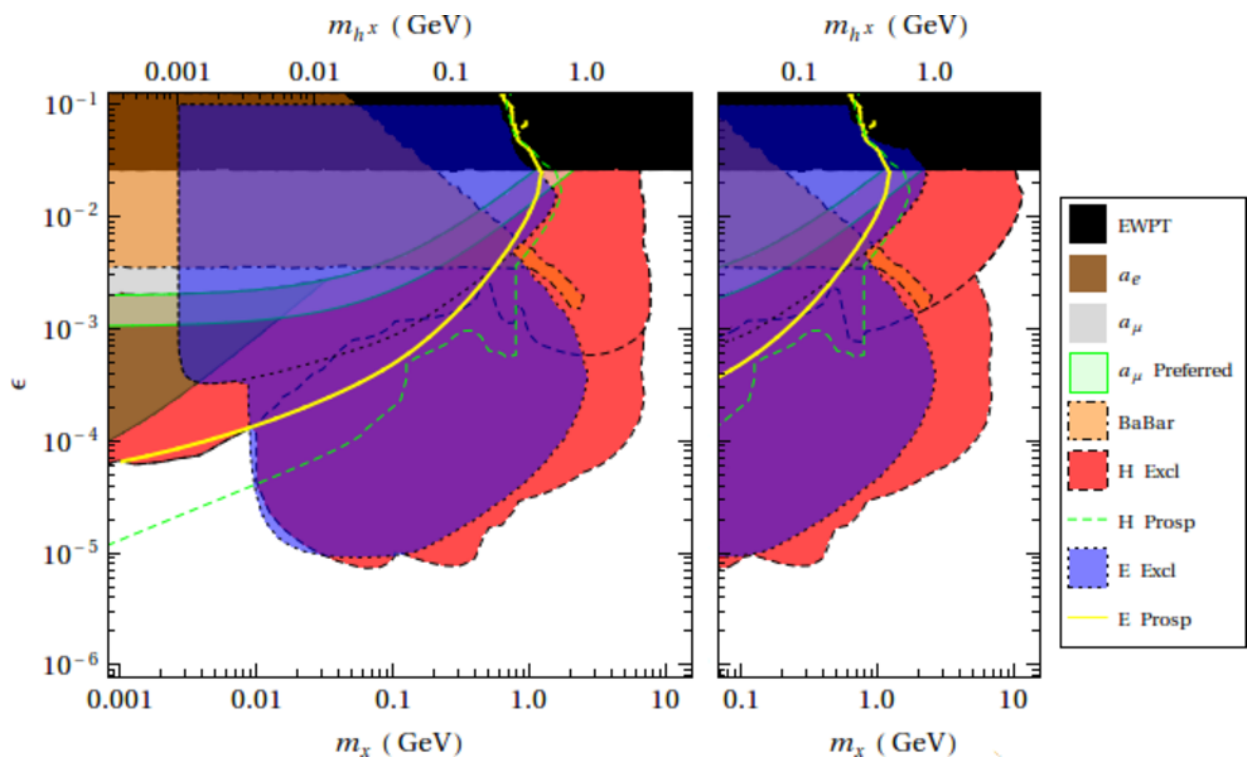

Figure 22. Composite plot of all non-cosmological limits we find for Case C. We divide the limits from electron and hadron beam dumps into two regions; the upper regions can be excluded based on $h_{1}^{x}$ decays alone, while the lower only on $A^{x}$ decays. Limits from invisible kaon decays are much weaker than the limits shown. If the limits from late decays discussed in section 3.3 are included, they rule out all masses $m_{x} \lesssim 1 \mathrm{GeV}$ except for a narrow region just above the $h_{1}^{x} \rightarrow \mu^{+} \mu^{-}$threshold. 


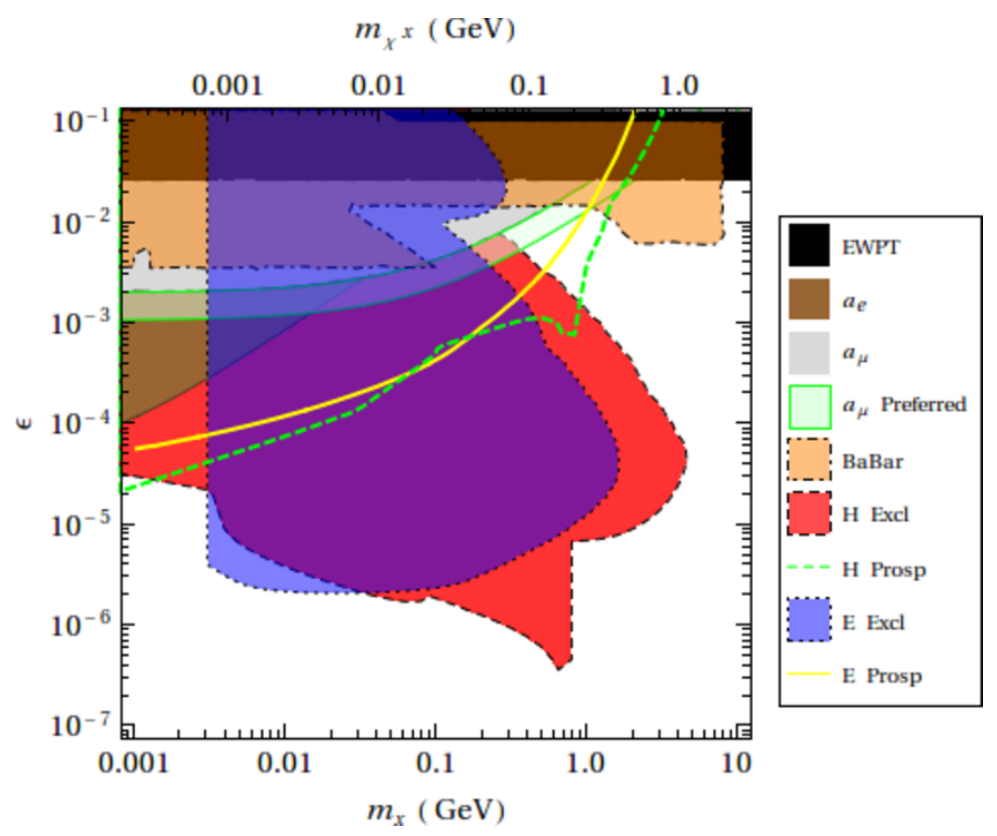

Figure 23. Composite plot of all non-cosmological limits we find for Case D. Limits from invisible kaon decays are much weaker than the limits shown. Including the limits from section 3.3 would exclude $m_{x} \lesssim 3 \mathrm{MeV}$, below which the decay $\chi_{2}^{x} \rightarrow \chi_{1}^{x} e^{+} e^{-}$is kinematically forbidden. However, their remains significant allowed parameter space at small $\epsilon$ even after this.

based solely on the hidden Higgs, while the lower are only excluded by the pseudoscalar decays. As discussed in section 4.5, the latter are more model-dependent and, in particular, can be avoided if $\mathrm{CP}$ is violated in the hidden sector. In either case, we find that both electron and proton experiments comfortably exclude the region of parameter space where this benchmark can explain $a_{\mu}$.

Finally, in Case D (figure 23), we find the first limits on hidden sectors where the vector decays to hidden fermions with visible decays. We have a diverse mix of limits from searches for visible and invisible final states, and reach to low values of $\epsilon$ thanks to the relatively short $\chi_{2}^{x}$ lifetime. Most interestingly, uniquely among our benchmarks this model has substantial parameter space still allowed where the $a_{\mu}$ discrepancy can be explained; this is due to the hidden Higgs decaying within the hidden sector. This region of parameter space will be probed by JLab and can be definitively excluded by INGRID.

The four parameter slopes considered in this paper cover the most likely experimental "phases" of the theory. Deviating away from these specific slopes will change the decay lifetimes of metastable states ( $\operatorname{such}$ as $A^{x}$ and $\chi_{2}^{x}$ ), but will not tend to alter the qualitative signatures. Thus, a similar set of experimental analyses are expected to be applicable to the general parameter space of the theory. The only exception would occur if the hidden vector had multiple significant hidden decay modes, i.e. to both hidden scalars and fermions. In this case, the limits we have found would still apply, but would be suppressed by branching ratios.

In summary, the model presented here strikes a convenient balance between simplicity and flexibility. From a supersymmetric standpoint, it is minimal. Despite this, it not only 
encompasses the most common hidden sectors previously considered, but it also extends those to situations with new and ineteresting phenomenology. For this reason, it serves as a convenient framework for studying general hidden sectors, including non-supersymmetric ones. We hope that the new hidden sector phenomenology observed here might encourage our experimental colleagues to perform dedicated searches for them.

\section{Acknowledgments}

We would like to thank Sonia Bacca, Nikita Blinov, Christopher Carone, Rouven Essig, Eder Izaguirre, Patrick de Niverville, Adam Ritz, Philip Schuster, Mark Sher, Michael Spannowsky, Natalia Toro, and Itay Yavin for useful discussions. We also thank an anonymous referee for their comments. This work was supported by the Australian Research Council and the National Sciences and Engineering Research Council of Canada (NSERC).

\section{A Masses and mixings in the hidden sector}

In this appendix we describe in more detail the masses and mixings within the minimal supersymmetric hidden sector formulated in refs. $[12,26]$ and investigated in this work. We also show how the hidden states connect to the visible MSSM sector, and we describe the calculation of the decay of $h_{1}^{x}$ to SM states.

\section{A.1 Masses for $\epsilon \rightarrow 0$}

In the absence of kinetic mixing, $\epsilon \rightarrow 0$, the hidden and visible sectors decouple and it is straightforward to obtain the mass eigenstates. The scalar components of $H$ and $H^{\prime}$ are assumed to develop real VEVs near or below the GeV scale,

$$
\langle H\rangle=\eta \sin \zeta, \quad\left\langle H^{\prime}\right\rangle=\eta \cos \zeta,
$$

with $\zeta \in[0, \pi / 2]$. As a result, the hidden vector $X_{\mu}$ obtains a mass equal to

$$
m_{x}=\sqrt{2} g_{x} \eta
$$

The remaining physical bosons are a pair of real scalars $h_{1}^{x}$ and $h_{2}^{x}$ (with $m_{h_{1}^{x}} \leq m_{h_{2}^{x}}$ ), and a pseudoscalar $A^{x}{ }^{4}$ In terms of the vector and pseudoscalar masses, the scalar mass matrix is ${ }^{5}$

$$
\mathcal{M}_{h^{x}}^{2}=\left(\begin{array}{cc}
m_{x}^{2} s_{\zeta}^{2}+m_{A^{x}}^{2} c_{\zeta}^{2} & -\left(m_{x}^{2}+m_{A^{x}}^{2}\right) s_{\zeta} c_{\zeta} \\
-\left(m_{x}^{2}+m_{A^{x}}^{2}\right) s_{\zeta} c_{\zeta} & m_{x}^{2} c_{\zeta}^{2}+m_{A^{x}}^{2} s_{\zeta}^{2}
\end{array}\right) .
$$

This matrix is identical in structure to the MSSM scalar Higgs mass matrix, and implies the tree-level relations

$$
m_{h_{1}^{x}} \leq m_{x}|\cos \zeta|, \quad \text { and } \quad m_{h_{1}^{x}} \leq m_{A^{x}}|\cos \zeta|
$$

\footnotetext{
${ }^{4}$ We do not consider $\mathrm{CP}$ violation in the present work.

${ }^{5}$ We will make use of the abbreviation, $c_{\zeta}\left(s_{\zeta}\right)=\cos \zeta(\sin \zeta)$.
} 
as well as

$$
m_{h_{1}^{x}}^{2}+m_{h_{2}^{x}}^{2}=m_{x}^{2}+m_{A^{x}}^{2} .
$$

We will also write

$$
\begin{aligned}
& H=\eta s_{\zeta}+\frac{1}{\sqrt{2}}\left(R_{1 a} h_{a}^{x}+i c_{\zeta} A^{x}\right), \\
& H^{\prime}=\eta c_{\zeta}+\frac{1}{\sqrt{2}}\left(R_{2 a} h_{a}^{x}+i s_{\zeta} A^{x}\right),
\end{aligned}
$$

where $R$ is the real orthogonal matrix that diagonalizes the masses.

Spontaneous symmetry breaking also mixes the $\mathrm{U}(1)_{x}$ gaugino with the fermion components of $H$ and $H^{\prime}$ to yield three hidden neutralinos $\chi_{1}^{x}, \chi_{2}^{x}, \chi_{3}^{x}$ (with $\left|m_{\chi_{1}^{x}}\right|<\left|m_{\chi_{2}^{x}}\right|<$ $\left.\left|m_{\chi_{3}^{x}}\right|\right)$. The entire spectrum of the theory can be specified by the seven input parameters $\left\{g_{x}, \mu^{\prime}, m_{x}, m_{A^{x}}, M_{x}, \tan \zeta, \epsilon\right\}$.

\section{A.2 Mixing with the MSSM}

When the kinetic mixing parameter $\epsilon$ is non-zero, the mixed term in eq. (2.2) induces kinetic mixing between hidden and visible gauge bosons and gauginos, as well as further mixing among the auxiliary fields. In components,

$$
\begin{aligned}
\mathscr{L} \supset & -\frac{1}{4} B^{\mu \nu} B_{\mu \nu}-\frac{1}{4} X^{\mu \nu} X_{\mu \nu}-\frac{\epsilon}{2 c_{W}} X^{\mu \nu} B_{\mu \nu} \\
& +\frac{1}{2} i \tilde{B}^{\dagger} \sigma \cdot \partial \tilde{B}+\frac{1}{2} i \tilde{X}^{\dagger} \sigma \cdot \partial \tilde{X}+\frac{\epsilon}{2 c_{W}} i \tilde{B}^{\dagger} \sigma \cdot \partial \tilde{X}+\frac{\epsilon}{2 c_{W}} i \tilde{X}^{\dagger} \sigma \cdot \partial \tilde{B} \\
& +\frac{1}{2} D_{B}^{2}+\frac{1}{2} D_{X}^{2}+\frac{\epsilon}{c_{W}} D_{X} D_{B} .
\end{aligned}
$$

where $c_{W}=\cos \theta_{W}$ refers to the weak mixing angle. The kinetic terms can be brought to their canonical form by making field redefinitions at the expense of generating new interactions among the visible and hidden sectors.

The gauge kinetic terms can be diagonalized to leading order in $\epsilon$ by the transformations

$$
\begin{aligned}
X_{\mu} & \rightarrow X_{\mu}+\epsilon t_{W} Z_{\mu}, \\
A_{\mu} & \rightarrow A_{\mu}-\epsilon X_{\mu}, \\
Z_{\mu} & \rightarrow Z_{\mu},
\end{aligned}
$$

where the fields $A_{\mu}$ and $Z_{\mu}$ are defined as in the SM, and $t_{W}$ is the tangent of the Weinberg angle. These transformations are not unique, but they have the advantage that the mass mixing induced between $Z$ and $X$ is very small, suppressed by both $\epsilon$ and $m_{x}^{2} / m_{Z}^{2}$, and has a negligible effect on the mass eigenvalues.

For the gauginos, neglecting a possible soft mass mixing term, the kinetic mixing is most cleanly removed by the redefinition ${ }^{6}$

$$
\begin{aligned}
\tilde{B} & \rightarrow \tilde{B}-\frac{\epsilon}{c_{W}} \tilde{X}, \\
\tilde{X} & \rightarrow \tilde{X},
\end{aligned}
$$

\footnotetext{
${ }^{6}$ No mass mixing is induced by gauge mediation, while the residual gravity-mediated contribution is expected to be suppressed relative to the $\tilde{X}$ mass by a factor of $\epsilon$ [12].
} 
to leading order in $\epsilon$. This transformation generates a coupling between the visible neutralinos and the hidden sector. The induced mass mixing between the visible and hidden sectors is again negligible, suppressed by both $\epsilon$ and the ratio of the hidden to visible neutralino masses.

Turning next to the scalars, the last term in eq. (A.7) mixes the hidden and visible $D$ terms. Let us represent the visible sector scalars by $\phi_{i}$ with hypercharge $Y_{i}$, and the hidden scalars by $\varphi_{j}=\left\{H, H^{\prime}\right\}$ with $\mathrm{U}(1)_{x}$ charge $x_{j}$. Upon integrating out the auxiliary fields, the $D$-term potential becomes [135]

$$
\begin{aligned}
V_{D}= & \frac{1}{2} c_{\epsilon}^{2} g_{Y}^{2}\left(\sum_{i} Y_{i}\left|\phi_{i}\right|^{2}\right)^{2}+\frac{1}{2} c_{\epsilon}^{2} g_{x}^{2}\left(\sum_{j} x_{j}\left|\varphi_{j}\right|^{2}\right)^{2} \\
& -s_{\epsilon} c_{\epsilon} g_{x} g_{Y}\left(\sum_{i} Y_{i}\left|\phi_{i}\right|^{2}\right)\left(\sum_{j} x_{j}\left|\varphi_{j}\right|^{2}\right)
\end{aligned}
$$

where $c_{\epsilon}=1 / \sqrt{1-\epsilon_{Y}^{2}}$ and $s_{\epsilon}=\epsilon_{Y} c_{\epsilon}$. The last term in this expression is precisely a Higgs portal interaction.

For $\eta \ll v$ (as we assume here) the modified $D$-term potential has a negligible effect on electroweak symmetry breaking, but it plays an important role in inducing symmetry breaking in the hidden sector. When the MSSM Higgs fields develop VEVs, driven almost entirely by the larger MSSM soft terms, an effective Fayet-Iliopoulos term is generated for $\mathrm{U}(1)_{x}$ which pushes the $H$ and $H^{\prime}$ fields to develop VEVs of their own. ${ }^{7}$

Expanding the visible and hidden Higgs fields about their VEVs, the Higgs portal interaction also induces a mass mixing between the visible and hidden mass eigenstates. This has only a very small effect on the mass eigenvalues, with deviations from the $\epsilon \rightarrow 0$ values suppressed by at least $\epsilon m_{x}^{2} / m_{z}^{2}$. For this reason it is an excellent approximation to separately diagonalise the scalars in the visible and hidden sectors, and treat the mixing as a mass insertion. To be explicit, we will assume that the visible Higgs sector has the form of the MSSM [27], with mass eigenstates $H^{ \pm}, A^{0}$, and

$$
\left(\begin{array}{c}
h^{0} \\
H^{0}
\end{array}\right)=\left(\begin{array}{rr}
\cos \alpha & -\sin \alpha \\
\sin \alpha & \cos \alpha
\end{array}\right)\left(\begin{array}{l}
\sqrt{2}\left[\operatorname{Re}\left(H_{u}^{0}\right)-v_{u}\right] \\
\sqrt{2}\left[\operatorname{Re}\left(H_{d}^{0}\right)-v_{d}\right]
\end{array}\right) .
$$

The mass mixing term is then ${ }^{8}$

$$
-\mathscr{L} \supset-\epsilon c_{\epsilon}^{2} t_{W} m_{x} m_{Z}\left[\sin (\alpha+\beta) h^{0}-\cos (\alpha+\beta) H^{0}\right]\left(s_{\zeta} R_{1 a}-c_{\zeta} R_{2 a}\right) h_{a}^{x}
$$

In the MSSM Higgs decoupling limit, $\alpha=\beta-\pi / 2$, this reduces to

$$
-\mathscr{L} \supset \epsilon c_{\epsilon}^{2} t_{W} m_{x} m_{Z}\left(s_{\zeta} R_{1 a}-c_{\zeta} R_{2 a}\right) h_{a}^{x}\left[\cos (2 \beta) h^{0}+\sin (2 \beta) H^{0}\right] .
$$

\section{A.3 Decays of the light scalar}

The $h_{1}^{x}$ scalar is the lightest $R$-even state in the hidden sector. When it is lighter than twice the mass of the lightest neutralino $\chi_{1}^{x}$, it will decay exclusively to the SM. The three leading

\footnotetext{
${ }^{7}$ In the absence of soft terms in the hidden sector, this would also cause the spontaneous breaking of supersymmetry in that sector [12].

${ }^{8}$ This corrects the original version of ref. [26].
} 


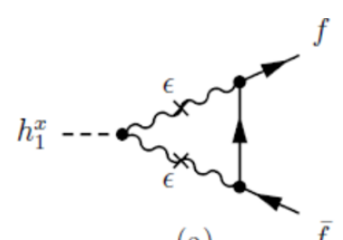

(a)

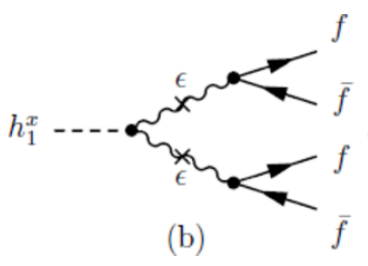

(b)

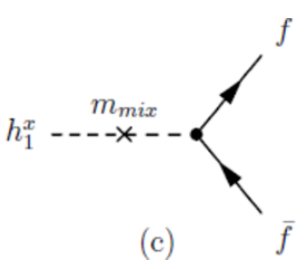

(c)

Figure 24. Three main contributions to the decay of the hidden sector Higgs: (a) loop decay through vector kinetic mixing; (b) four-body decay through vector kinetic mixing; (c) two-body decay through $D$-term-induced mass mixing.

contributions to the decay width are shown in figure 24. The first two are discussed in detail in [17], and require a pair of insertions of $\epsilon$ in the amplitudes as well as the coupling of the hidden scalar to the hidden vector,

$$
\mathscr{L} \supset g_{x} m_{x} h_{a}^{x} X_{\mu} X^{\mu}\left(s_{\zeta} R_{1 a}+c_{\zeta} R_{2 a}\right) .
$$

The third contribution to the decay comes from the Higgs portal interaction of eq. (A.12) [26]. This piece has only one power of $\epsilon$ in the amplitude, but also receives suppression by $m_{x}^{2} / m_{Z}^{2}$ and small SM Yukawa couplings. In the absence of interference with the loop-mediated two-body channel and working in the MSSM decoupling limit, the Higgs-portal-induced decay width is

$$
\Gamma\left(h_{1}^{x}\right)=\epsilon^{2} t_{W}^{2} c_{2 \beta}^{2}\left(s_{\zeta} R_{1 a}-c_{\zeta} R_{2 a}\right)^{2}\left(\frac{m_{x} m_{Z}}{m_{h}^{2}}\right)^{2} \Gamma\left(h_{S M}^{0}, m_{h}=m_{h_{1}^{x}}\right),
$$

where $\Gamma\left(h_{S M}^{0}, m_{h}=m_{h_{1}^{x}}\right)$ is the width the SM Higgs would have if its mass were equal to $m_{h_{1}^{x}}$. The branching fractions will also follow those of a SM Higgs with $m_{h}=m_{h_{1}^{x}}$. Decays through mixing with the heavier Higgs will generally be suppressed unless $\tan \beta$ is close to unity, when the mixing with $h^{0}$ vanishes.

In figure 25 we plot the total decay length $c \tau$ of $h_{1}^{x}$ as a function of its mass. For this plot, we use $\epsilon=10^{-3}$ and the hidden sector benchmark C, together with the decoupling and large $\tan \beta$ limit of the MSSM. The results for benchmarks A and B are qualitatively similar, while for benchmark $\mathrm{D}$ the scalar decays promptly to the hidden sector, $h_{1}^{x} \rightarrow \chi_{1}^{x} \chi_{1}^{x}$. This result includes the interference between the loop and Higgs-portal contributions to the amplitude. We also show in figure 25 lines corresponding to what the decay length would be with the Higgs-portal alone or with the loop-induced vector-mediated decay alone.

For $2 m_{\pi}<m_{h_{1}^{x}} \lesssim 1 \mathrm{GeV}$ there is a significant uncertainty in the Higgs width to hadronic modes. For the mass mixing contribution, we use the parametrization of ref. [136] to compute the total rate and the branching fractions. A comparison of this and other estimates is given in ref. [45] (see also [137, 138]). For the loop diagram, we do not compute any contribution for hadronic decays below $\Lambda_{Q C D} \approx 2.5 \mathrm{GeV}$; this represents an additional theoretical uncertainty.

We would like to estimate the size of this uncertainty, and its corresponding effect on the limits we extract in sections 4 and 5 . When we have no mass mixing, our limits are surprisingly robust to the omission of any hadronic decay modes below $\Lambda_{Q C D}$. The upper 


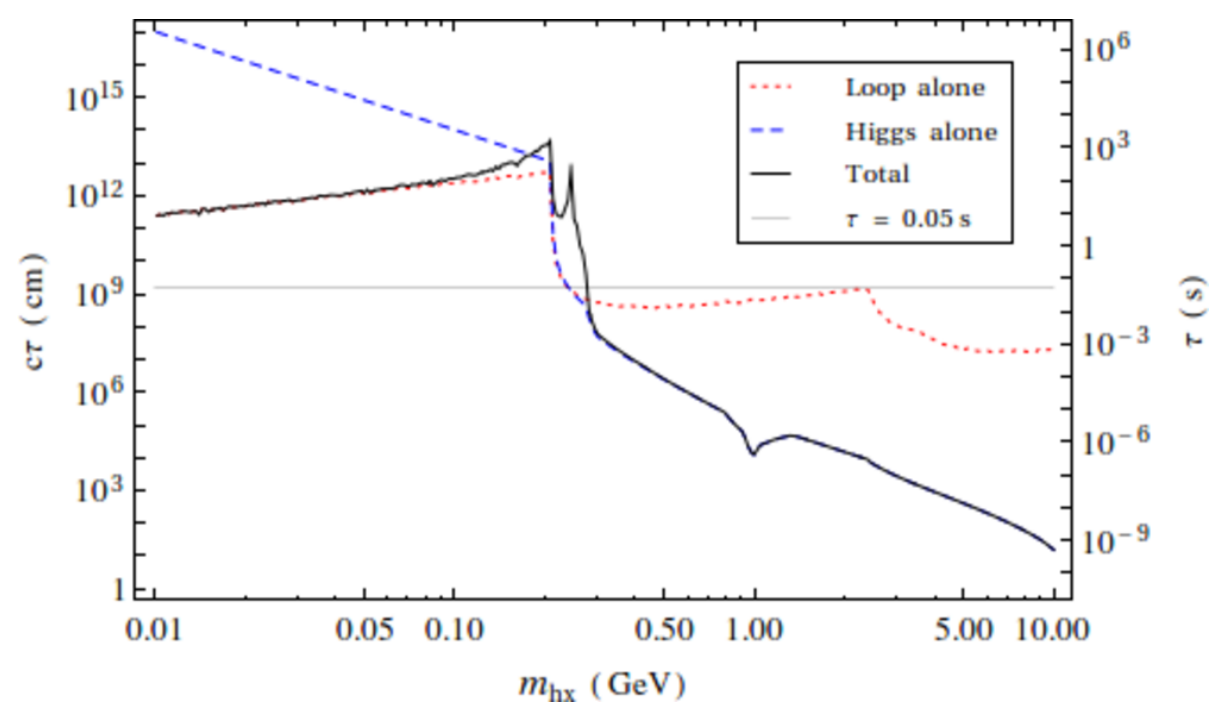

Figure 25. Decay length $(c \tau)$ for a hidden sector Higgs $h_{1}^{x}$ for different decay mechanisms. In making this figure, we have fixed $\epsilon=10^{-3}$, $\tan \beta \rightarrow \infty$, and the other parameters as in benchmark C. The horizontal line shows $\tau=0.05 \mathrm{~s}$, relevent for cosmological constraints as discussed in the text.

bounds on $\epsilon$ we find in this region of parameter space come when $c \tau_{h_{1}^{x}} \sim 10^{4} \mathrm{~m}$. This is large compared to the typical size of experiments, especially once a boost of $\gamma \sim 10-100$ is included. The decay probability eq. (4.20) is then

$$
P_{\mathrm{dec}} \sim \frac{L_{\mathrm{dec}}}{\gamma c \tau} \propto \Gamma
$$

Increasing the total width will increase the number of hidden states decaying within our detector. This is offset by the fact that the extra width is to hadrons, while all searches tagged on leptons. We thus pay a branching ratio penalty $\propto 1 / \Gamma$, cancelling the factor from $P_{\text {dec }}$.

This argument holds provided that the width does not increase too much, specifically that the approximation (A.16) is valid. This is why our exclusions change with the inclusion of mass mixing; those limits are set for parameters where the scalar lifetime $c \tau_{h_{1}^{x}} \sim 10$ $100 \mathrm{~m}$. Consequently, those limits are more sensitive to uncertainties in the hidden Higgs width. The sensitivity is greatest to increases in the width, which would suppress signals by having more decays within the shield. Decreasing the width would make the limits stronger, approaching the exclusions without mass mixing. We note that our choice for the Higgs hadronic width is both the most recent and among the largest of those discussed in ref. [45], suggesting the main theoretical uncertainty makes our limits conservative.

\section{B Details of hidden vector production at electron beam dumps}

In section 4, we sketched the calculation of the number of hidden sector events at an electron beam dump. Here we provide some of the details omitted there. 


\section{B.1 Effective flux}

We begin by considering the effective flux $\chi$, introduced in eq. (4.2) that contains the form factors describing the details of the target. This quantity was defined in ref. [75] as

$$
\chi=\frac{1}{2 M_{i}} \int_{t_{\min }}^{t_{\max }} d t \int_{M_{i}^{2}}^{M_{u p}^{2}} d M_{f}^{2} \frac{1}{t^{2}}\left(2 t_{\mathrm{aux}} W_{1}\left(t, M_{f}^{2}\right)+\left(t-t_{\min }\right) W_{2}\left(t, M_{f}^{2}\right)\right) .
$$

Here, $t=-q^{2}$ ranges between $t_{\min }=\left(m_{x} / 2 E_{e}\right)^{2}$ and $t_{\max }=m_{x}^{2} ; M_{f}$ is the mass of the target final state, and ranges between $M_{i}$ and $M_{u p}^{2} \approx M_{i}\left(M_{i}+2 E_{e}\right) . W_{1,2}$ are form factors describing the target and including both elastic and inelastic behaviour, and $t_{\text {aux }} \equiv$ $\left(p_{e} \cdot k_{X}-\frac{1}{2} m_{x}^{2}\right) /\left(E_{e}-E_{x}\right)$.

We use analytic approximations for the form factors taken from refs. [75, 139]. These are given in terms of the partially integrated quantities $G$ :

$$
G_{1,2}(t)=\frac{1}{2 M_{i}} \int_{M_{i}^{2}}^{M_{u p}^{2}} d M_{f}^{2} W_{1,2}\left(t, M_{f}^{2}\right) .
$$

The form factor $W_{1}$ has a negligible contribution to hidden vector production [19]. We split $G_{2}$ into a sum of elastic and inelastic contributions, which in turn are the product of atomic and nuclear contributions. The elastic term is

$$
G_{2}^{e l}(t)=\left(\frac{a^{2} t}{1+a^{2} t}\right)^{2}\left(\frac{1}{1+t / d}\right)^{2} Z^{2}
$$

The parameters $a$ and $d$ are well approximated as $a \approx 111 /\left(m_{e} \sqrt[3]{Z}\right)$ and $d \approx$ $0.164 \mathrm{GeV}^{2} / \sqrt[3]{A^{2}}$, with $Z$ and $A$ the usual atomic and mass numbers. The terms in the first (second) brackets describe the effects of atomic screening (finite nuclear size).

For the inelastic term we take, based on ref. [139],

$$
G_{2}^{i n e l}=\left(\frac{a^{\prime 2} t}{1+a^{\prime 2} t}\right)^{2} C(t)\left[Z G_{2 p}^{e l}+(A-Z) G_{2 n}^{e l}\right] .
$$

The first set of brackets again describes the atomic screening factor, with $a^{\prime} \approx$ $773 /\left(m_{e} \sqrt[3]{Z^{2}}\right)$. The remaining terms describe the inelastic nuclear form factors, in terms of a prefactor $C$ and nucleon functions $G_{2 p, n}^{e l}$. The prefactor is

$$
C(t)= \begin{cases}1 & \text { if } Q>2 P_{F}=0.5 \mathrm{GeV} \\ \frac{3 Q}{4 P_{F}}\left[1-\frac{Q^{2}}{12 P_{F}^{2}}\right] & \text { otherwise }\end{cases}
$$

with $Q$ defined by

$$
Q=\frac{t^{2}}{4 m_{p}^{2}}+t
$$

while the nucleon functions are

$$
\begin{aligned}
G_{2 p}^{e l} & =\frac{m_{p}}{M_{i}} \frac{1}{\left(1+t / t_{0}\right)^{4}} \frac{1+2.79^{2} \tau}{1+\tau}, \\
G_{2 n}^{e l} & =\frac{m_{p}}{M_{i}} \frac{1}{\left(1+t / t_{0}\right)^{4}} \frac{1.91^{2} \tau}{1+\tau},
\end{aligned}
$$

where $t_{0}=0.71 \mathrm{GeV}^{2}$ and $\tau=t /\left(4 m_{p}^{2}\right)$. 


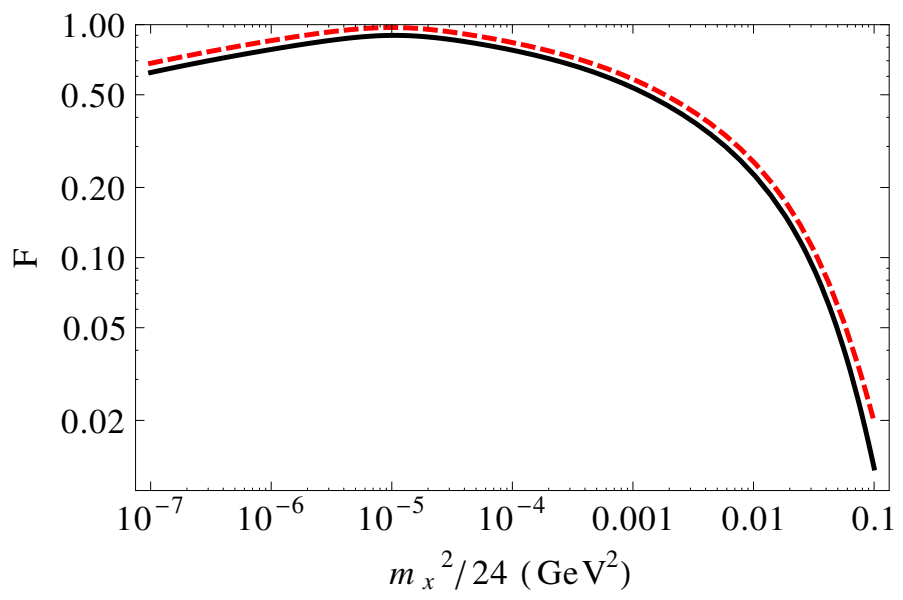

Figure 26. The scaled flux $F$ as a function of $q_{\min }=m_{x}^{2} /(2 E)$ for an electron beam of energy $E=$ $12 \mathrm{GeV}$ impacting an aluminium target. The black, solid line represents our choice of form factors, while the red, dashed line corresponds to that of e.g. ref. [22] (compare figure 4 of that reference).

Note that refs. [19, 22, 67] used a different form for $G_{2}^{\text {inel }}$, specifically the expressions given for elastic scattering from protons in ref. [75]. The difference between the two choices is small, as the elastic contribution to $G_{2}$ is dominant. We compare the two in figure 26 using the scaled flux

$$
F=\frac{4}{3} \alpha^{3} \chi \frac{X_{0} N_{0}}{A m_{e}^{2}}
$$

introduced in ref. [22]. The difference is only a few percent for the mass range of interest, with our parameterisation the smaller of the two.

\section{B.2 Off-shell production}

The general process for hidden sector production through an off-shell vector is shown in figure 27 (a simple generalisation of figure 7). We can write the matrix element as

$$
\mathcal{M}_{2 \rightarrow n+2}=\frac{1}{q^{2}} T_{\mu}^{N}\left(p_{N}, k_{N^{\prime}}\right) S_{e}^{\mu \nu}\left(p_{e}, q, k_{e}\right) N_{\nu}^{x}\left(k_{1}, \ldots k_{n}\right),
$$

with $T^{N}, S_{e}$ and $N^{x}$ respectively the target fermion line trace, the electron line trace, and the terms related to the hidden sector. Squaring this and integrating over the $k_{N^{\prime}}$ phase space lets us introduce form factors:

$$
\begin{aligned}
\int d \Pi_{n+2} \frac{1}{2 N_{i}} \sum\left|\mathcal{M}_{2 \rightarrow n+2}\right|^{2} & =\int\left(\prod_{i=1}^{n} \frac{d^{3} k_{i}}{(2 \pi)^{3} 2 E_{i}}\right) \frac{d^{3} k_{e}}{(2 \pi)^{3} 2 E_{e f}} \frac{e^{2}}{\left(q^{2}\right)^{2}} 2 \pi M_{i} \\
\times & \left\{-W_{1} \eta_{\mu \nu}+W_{2} \frac{p_{N \mu} p_{N \nu}}{M_{i}^{2}}\right\} \sum S_{e}^{\mu \rho}\left(S_{e}^{\nu \sigma}\right)^{*} N_{\rho}^{x}\left(N_{\sigma}^{x}\right)^{*}
\end{aligned}
$$

with $M_{i}$ the target mass and $E_{\text {ef }}$ the final state electron energy.

To generalise the WW approximation, we follow ref. [75] in rewriting the phase space integral over $k_{e}$ into an integral over $M_{f}^{2}, t=-q^{2}$ and an angular degree of freedom $\phi_{S}$. 


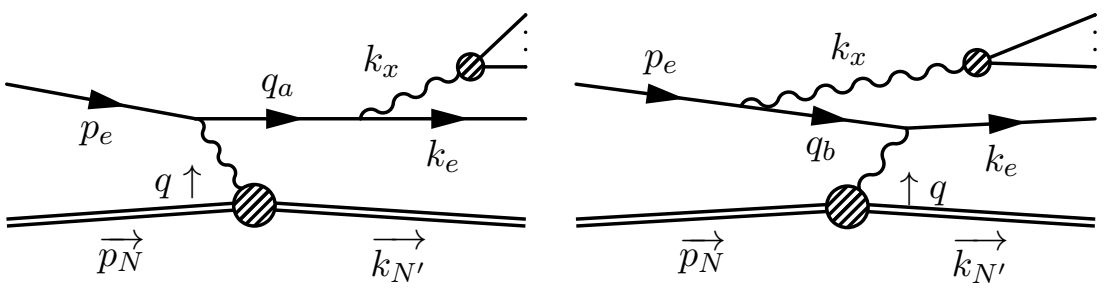

Figure 27. The two leading Feynman diagrams for production of off-shell massive vector in electron-fixed target scattering. The unlabelled lines represent the $n$ hidden sector states ultimately produced through the hidden vector.

This is done by making use of a special frame $S$, defined as the frame where $\vec{u} \equiv \vec{p}_{e}+\vec{p}_{N}-\vec{k}_{x}$ vanishes. Specifically, $\phi_{S}$ is the azimuthal angle of $\vec{k}_{e}$ relative to $\vec{p}_{e}-\vec{k}_{x}$ in this frame. We also note that the hidden sector factors $N$ have no dependence on $k_{e}$, while the form factors depend only on $t$ and $M_{f}^{2}$. This brings us to the following expression for the integrated matrix element:

$$
\begin{aligned}
& \int \frac{d \Pi_{n+2}}{2 N_{i}} \sum\left|\mathcal{M}_{2 \rightarrow n+2}\right|^{2}=\frac{\alpha}{4} \int\left(\prod_{i=1}^{n} \frac{d^{3} k_{i}}{(2 \pi)^{3} 2 E_{i}}\right) \sum N_{\rho}^{x}\left(N_{\sigma}^{x}\right)^{*}\left(k_{1}, \ldots k_{n}\right) \\
& \times \frac{M_{i}}{\sqrt{\left(u \cdot p_{N}\right)^{2}-M_{i}^{2} u^{2}}} \int d M_{f}^{2} d t \frac{1}{t^{2}}\left\{-W_{1} \eta_{\mu \nu}+W_{2} \frac{p_{N \mu} p_{N \nu}}{M_{i}^{2}}\right\} \\
& \times \int \frac{d \phi_{S}}{2 \pi} \sum S_{e}^{\mu \rho}\left(S_{e}^{\nu \sigma}\right)^{*}\left(p_{e}, k_{x}, M_{f}^{2}, t, \phi_{S}\right) .
\end{aligned}
$$

Note that the only difference so far with the derivation of ref. [75] is that in that case, the integral over $k_{1} \cdots k_{n}$ is replaced by a single integral over $k_{x}$, and the function $N_{\rho}^{x}\left(N_{\sigma}^{x}\right)^{*}$ is replaced by $-\eta_{\rho \sigma}$.

At this point, we sketch the derivation of eq. (4.7). We introduce a trivial integral over $k_{x}$ using a delta function. This lets us rewrite the integral over $N_{\rho}^{x}\left(N_{\sigma}^{x}\right)^{*}$ in terms of the vector partial decay widths:

$$
\begin{aligned}
& \int\left(\prod_{i=1}^{n} \frac{d^{3} k_{i}}{(2 \pi)^{3} 2 E_{i}}\right) \sum N_{\rho}^{x}\left(N_{\sigma}^{x}\right)^{*}\left(k_{1}, \ldots k_{n}\right) \\
& \quad=\int\left(\prod_{i=1}^{n} \frac{d^{3} k_{i}}{(2 \pi)^{3} 2 E_{i}}\right) \frac{d^{4} k_{x}}{(2 \pi)^{4}}(2 \pi)^{4} \delta^{(4)}\left(k_{x}-k_{1}-\cdots k_{n}\right) \sum N_{\rho}^{x}\left(N_{\sigma}^{x}\right)^{*} \\
& \quad=\int \frac{d^{4} k_{x}}{(2 \pi)^{4}} \frac{1}{\left(k_{x}^{2}-m_{x}^{2}\right)^{2}}\left(A\left(k_{x}^{2}\right) \eta_{\rho \sigma}+B\left(k_{x}^{2}\right) k_{x \rho} k_{x \sigma}\right) .
\end{aligned}
$$

The last term vanishes when contracted with $S_{e}^{\mu \rho}$ via the QED Ward identity, while

$$
A\left(k_{x}^{2}\right)=-2 \sqrt{k_{x}^{2}} \Gamma_{x}\left(k_{x}^{2}\right),
$$

with $\Gamma_{x}$ the partial width for a vector of mass $m_{x}^{2}=k_{x}^{2}$ to decay to the relevant hidden sector final state. From this, the usual WW argument leads to eq. (4.7). 
The WW approximation is a Taylor expansion of the (integral of the) electron line trace about the minimal momentum transfer $t_{\min }$, leading to the two approximations

$$
\int \frac{d \phi_{S}}{2 \pi} \sum S_{e}^{\mu \nu}\left(S_{e \mu \nu}\right)^{*}\left(p_{e}, k_{x}, M_{f}^{2}, t, \phi_{S}\right) \approx \sum S_{e}^{\mu \nu}\left(S_{e \mu \nu}\right)^{*}\left(p_{e}, k_{x}, M_{i}^{2}, t_{\min }\right),
$$

and

$$
\begin{aligned}
-\int \frac{d \phi_{S}}{2 \pi} \frac{p_{N \mu} p_{N \nu}}{M_{i}^{2}} \sum S_{e}^{\mu \rho}\left(S_{e \rho}^{\nu}\right)^{*}\left(p_{e},\right. & \left.k_{x}, M_{f}^{2}, t, \phi_{S}\right) \\
& \approx \frac{t-t_{\min }}{2 t_{\mathrm{aux}}} \sum S_{e}^{\mu \nu}\left(S_{e \mu \nu}\right)^{*}\left(p_{e}, k_{x}, M_{i}^{2}, t_{\min }\right) .
\end{aligned}
$$

As noted before, $\overrightarrow{q^{*}}$ is parallel to $\vec{p}_{e}-\vec{k}_{x}$. It follows that $\vec{k}_{e}$ is also parallel to $\vec{p}_{e}-\vec{k}_{x}$, allowing us to omit $\phi_{S}$ from the right-hand side of eqs. (B.14) and (B.15). This approximation can be checked using the explicit expressions for $S_{e}^{\mu \nu}$. It follows from the presence of the collinear pole when the electron is nearly on-shell, as well as the photon propagator that results in the dominance of processes where $t \approx t_{\min }$.

The equivalent approximations for the off-shell case are easy to guess:

$$
\int \frac{d \phi_{S}}{2 \pi} \sum S_{e}^{\rho \mu}\left(S_{e \rho}^{\nu}\right)^{*}\left(p_{e}, k_{x}, M_{f}^{2}, t, \phi_{S}\right) \approx \sum S_{e}^{\rho \mu}\left(S_{e \rho}^{\nu}\right)^{*}\left(p_{e}, k_{x}, M_{i}^{2}, t_{\min }\right),
$$

and

$$
\begin{aligned}
-\int \frac{d \phi_{S}}{2 \pi} \frac{p_{N \mu} p_{N \nu}}{M_{i}^{2}} \sum S_{e}^{\mu \rho}\left(S_{e}^{\nu \sigma}\right)^{*}\left(p_{e},\right. & \left.k_{x}, M_{f}^{2}, t, \phi_{S}\right) \\
& \approx \frac{t-t_{\min }}{2 t_{\mathrm{aux}}} \sum S_{e}^{\rho \mu}\left(S_{e \rho}\right)^{*}\left(p_{e}, k_{x}, M_{i}^{2}, t_{\min }\right) .
\end{aligned}
$$

Once these are put into eq. (B.11), eq. (4.8) is immediate. The validity of these approximations can also be checked by direct computation. We comment on a few points in the derivation. The easiest way to analyse these expressions is to expand in a tensor basis that respects the QED Ward identity. For example, the tensor in eq. (B.16) can only depend on $p_{e}, q^{*}, k_{x}$ and the metric, so we may define four functions $f_{i}$ through

$$
\int \frac{d \phi_{S}}{2 \pi} \sum S_{e}^{\rho \mu}\left(S_{e \rho}{ }^{\nu}\right)^{*}\left(p_{e}, k_{x}, M_{f}^{2}, t, \phi_{S}\right)=\sum_{i} f_{i}\left(p_{e}, k_{x}, M_{f}^{2}, t\right) L_{i}^{\mu \nu},
$$

with (for example)

$$
\begin{aligned}
L_{1}^{\mu \nu} & =\frac{k_{x}^{2}}{p_{e} \cdot k_{x}} p_{e}^{\mu} p_{e}^{\nu}+p_{e} \cdot k_{x} \eta^{\mu \nu}-p_{e}^{\mu} k_{x}^{\nu}-k_{x}^{\mu} p_{e}^{\nu}, \\
L_{2}^{\mu \nu} & =\frac{k_{x}^{2}}{q^{*} \cdot k_{x}} q^{* \mu} q^{* \nu}+q^{*} \cdot k_{x} \eta^{\mu \nu}-q^{* \mu} k_{x}^{\nu}-k_{x}^{\mu} q^{* \nu}, \\
L_{3}^{\mu \nu} & =\left(p_{e} \cdot k_{x} q^{* \mu}-q^{*} \cdot k_{x} p_{e}^{\mu}\right)(\mu \rightarrow \nu), \\
L_{4}^{\mu \nu} & =k_{x}^{2} \eta^{\mu \nu}-k_{x}^{\mu} k_{x}^{\nu} .
\end{aligned}
$$


The approximation of eq. (B.16) is then no more than replacing the functions $f_{i}$ of eq. (B.18) by their values at the minimal kinematics $t=t_{\min }, M_{f}^{2}=M_{i}^{2}$. We know that these functions are finite and non-zero at this point, as it corresponds to the physical point for photon-electron scattering; while the factor of $t^{-2}$ in eq. (B.11) leads the contribution from this region to generically dominate.

The approximation of eq. (B.17) is slightly more complicated. The tensor expansion is naïvely more involved, as we also have a possible dependence on $p_{N}$. However, because $\overrightarrow{q^{*}}$ is parallel to $\vec{p}_{e}-\vec{k}_{x}$, we can rewrite terms involving $p_{N}^{\mu}$ in terms of $p_{e}^{\mu}-k_{x}^{\mu}$ and $q^{* \mu}$; the basis $L_{i}^{\mu \nu}$ is still sufficient. So we have the expansion

$$
\int \frac{d \phi_{S}}{2 \pi} \frac{p_{N \mu} p_{N \nu}}{M_{i}^{2}} \sum S_{e}^{\mu \rho}\left(S_{e}^{\nu \sigma}\right)^{*}\left(p_{e}, k_{x}, M_{f}^{2}, t, \phi_{S}\right)=\sum_{i} g_{i}\left(p_{e}, k_{x}, M_{f}^{2}, t\right) L_{i}^{\mu \nu} .
$$

The WW approximation involves using not the first term in the Taylor expansion of the $g_{i}$, but the second. The leading term is suppressed; specifically, it is not singular when the intermediate electron goes on-shell. The term linear in $t$ is singular in that limit, so is the first term we keep.

The final step is to relate the linear terms in eq. (B.19) and the leading terms in eq. (B.18). It is clear that eq. (B.17) will hold if

$$
g_{i}(t)=g_{i}\left(t_{\min }\right)+\frac{t-t_{\min }}{2 t_{\text {aux }}} f_{i}(t)+\mathcal{O}\left(t-t_{\text {min }}\right)^{2} .
$$

This identification only holds approximately, to leading order in small parameters $\delta / E$ where

$$
E \in\left\{E_{e}, E_{x}, E_{e}-E_{x}\right\} \quad \text { and } \quad \delta \in\left\{\sqrt{t_{\mathrm{aux}}}, \frac{M_{f}^{2}-M_{i}^{2}}{2 M_{i}}, \frac{m_{e}^{2}}{\sqrt{t_{\mathrm{aux}}}}, \frac{k_{x}^{2}}{\sqrt{t_{\mathrm{aux}}}}\right\} .
$$

The assumption that $\delta / E$ is small is also part of the WW approximation.

There is one further non-trivial point, which is that in the basis we have chosen the function $g_{3}$ does not actually satisfy eq. (B.20). This is not an issue because when this term is contracted with $N_{\rho}^{x}\left(N_{\sigma}^{x}\right)^{*}$, it always gives to a subleading contribution in $\delta / E$. Since $N_{\rho}^{x}\left(N_{\sigma}^{x}\right)^{*}$ can be expanded in terms of the metric and the hidden state momenta $k_{i}$, we need consider two contractions. The trace terms are

$$
\begin{array}{rlrl}
\eta_{\mu \nu} g_{1} L_{1}^{\mu \nu} & \sim \frac{1}{t_{\mathrm{aux}}} & \eta_{\mu \nu} g_{2} L_{2}^{\mu \nu} \sim \frac{1}{t_{\mathrm{aux}}}, \\
\eta_{\mu \nu} g_{3} L_{3}^{\mu \nu} \sim \frac{1}{\sqrt{t_{\mathrm{aux}}}} & \eta_{\mu \nu} g_{4} L_{4}^{\mu \nu} \sim \frac{k_{x}^{2}}{t_{\mathrm{aux}}^{3 / 2}},
\end{array}
$$

and the third term is suppressed relative to the first two. For the contraction with terms of the form $k_{i \mu} k_{j \nu}$, note that in general the angular separation of the hidden state terms is large compared to the separation between $\vec{p}_{e}$ and $\vec{k}_{x}$; so terms of the form $\left\{p_{e}, q^{*}, k_{x}\right\} \cdot k_{i}$ will not be small. Representing such scalar products by $\Lambda$, we have

$$
\begin{array}{rlrl}
k_{i \mu} k_{j \nu} g_{1} L_{1}^{\mu \nu} & \sim \Lambda^{2} & & k_{i \mu} k_{j \nu} g_{2} L_{2}^{\mu \nu} \sim \Lambda^{2}, \\
k_{i \mu} k_{j \nu} g_{3} L_{3}^{\mu \nu} \sim \Lambda^{2} t_{\mathrm{aux}} & k_{i \mu} k_{j \nu} g_{4} L_{4}^{\mu \nu} \sim \Lambda^{2} .
\end{array}
$$

Again, the third term is suppressed compared to the others. It follows that the approximation of eq. (B.17) is valid within the WW assumptions. 


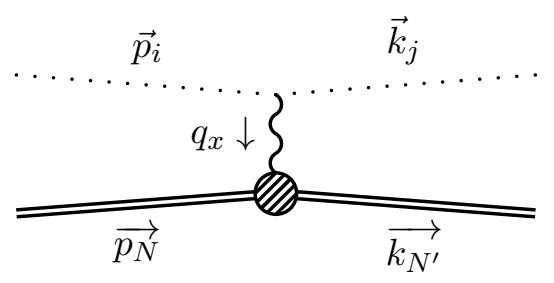

Figure 28. Dominant scattering process between visible and hidden sectors. The dotted lines represent various possible hidden sector states as discussed in the text, while the solid lines are either nucleons or electrons.

\section{B.3 Vector decay widths}

The partial widths for a vector to decay to hidden states, used in sections 4 and 5 , are given here. For the Higgsstrahlung "decay", we have

$$
\Gamma_{H a}\left(k_{x}^{2}\right)=\frac{\alpha_{x} N_{H, a}^{2}}{12} \sqrt{k_{x}^{2}} \sqrt{I\left(\mu_{a}^{2}, \mu_{x}^{2}\right)}\left[I\left(\mu_{a}^{2}, \mu_{x}^{2}\right)+12 \mu_{x}^{2}\right]
$$

for the scalar final states

$$
\Gamma_{S a}\left(k_{x}^{2}\right)=\frac{\alpha_{x} N_{S, a}^{2}}{12} \sqrt{k_{x}^{2}}\left\{I\left(\mu_{a}^{2}, \mu_{A^{x}}^{2}\right)\right\}^{3 / 2} ;
$$

and for decays to fermions,

$$
\begin{aligned}
\Gamma_{F i j}\left(k_{x}^{2}\right)=\frac{1}{3} \alpha_{x}\left|N_{F, i j}\right|^{2} & \left(1-\frac{1}{2} \delta_{i j}\right) \sqrt{k_{x}^{2}} \sqrt{I\left(\mu_{i}^{2}, \mu_{j}^{2}\right)} \\
& \times\left\{1-\frac{1}{2}\left(\mu_{i}^{2}+\mu_{j}^{2}\right)-\frac{1}{2}\left(\mu_{i}^{2}-\mu_{j}^{2}\right)^{2}-3 \mu_{i} \mu_{j} \frac{\Re N_{F, i j}^{2}}{\left|N_{F, i j}\right|^{2}}\right\} .
\end{aligned}
$$

The expressions for the actual decay widths derive from eqs. (B.25) and (B.26) with the replacement $k_{x}^{2} \rightarrow m_{x}^{2}$. The triangle function is

$$
I(a, b)=1-2 a-2 b+a^{2}+b^{2}-2 a b ;
$$

the kinematic factors are

$$
\mu_{x}^{2}=\frac{m_{x}^{2}}{k_{x}^{2}}, \quad \mu_{a}^{2}=\frac{m_{h_{a}^{x}}^{2}}{k_{x}^{2}}, \quad \mu_{A^{x}}^{2}=\frac{m_{A^{x}}^{2}}{k_{x}^{2}}, \quad \mu_{i}^{2}=\frac{m_{\chi_{i}^{x}}^{2}}{k_{x}^{2}}
$$

and the mixing matrix functions are as defined in eq. (4.13).

\section{Hidden sector-visible sector scattering}

In this appendix we provide relevant details on hidden sector-visible sector scattering. We are interested in the $2 \rightarrow 2$ processes shown in figure 28, with the two sectors coupled through a $t$-channel $Z^{x}$. The dotted lines represent one of a number of possible initial and final hidden states: $\chi_{i}^{x} \rightarrow \chi_{j}^{x}$ (with both $i=j$ and $i \neq j$ ); $A^{x} \rightarrow h_{a}^{x}$; and $h_{a}^{x} \rightarrow A^{x}$ or $Z^{x}$. The visible sector particles can be either nucleons or electrons. 
We define $Q^{2} \equiv-q_{x}^{2}>0$. Additionally, $m_{i}\left(m_{f}\right)$ are the initial (final) hidden sector masses; and $E_{i}, p_{i}$ the initial hidden sector energy and momentum. The differential cross sections are

$$
\frac{d \sigma_{\mathrm{HS}}}{d Q^{2}}=\frac{4 \pi \alpha \alpha_{x} \epsilon^{2}}{p_{i}^{2}} \frac{1}{\left(Q^{2}+m_{x}^{2}\right)^{2}} \frac{1}{4 m_{N}^{2}} \mathcal{I}_{\mathrm{HS}}\left(Q^{2}\right) .
$$

The functions $\mathcal{I}_{\mathrm{HS}}$ are, for fermion scattering:

$$
\begin{aligned}
\mathcal{I}_{F, i j}=\mid & \left.N_{F, i j}\right|^{2}\left[\left(2 E_{i} m_{N}-\frac{1}{2}\left(Q^{2}-m_{i}^{2}+m_{f}^{2}\right)\right)^{2}\left(F_{1, N}^{2}+\frac{Q^{2}}{4 m_{N}^{2}} F_{2, N}^{2}\right)\right. \\
& \left.-\left(m_{i}^{2} Q^{2}+\frac{1}{4}\left(Q^{2}-m_{i}^{2}+m_{f}^{2}\right)^{2}\right)\left(F_{1, N}+F_{2, N}\right)^{2}\right] \\
& -\left(\left|N_{F, i j}\right|^{2} Q^{2}+\left|N_{F, i j} m_{i}+N_{F, i j}^{*} m_{f}\right|^{2}\right)\left[-3 Q^{2}\left(F_{1, N}+F_{2, N}\right)^{2}\right. \\
& \left.+\left(Q^{2}+4 m_{N}^{2}\right)\left(F_{1, N}^{2}+\frac{Q^{2}}{4 m_{N}^{2}} F_{2, N}^{2}\right)\right]
\end{aligned}
$$

for a pseudoscalar initial or final state:

$$
\begin{aligned}
\mathcal{I}_{S, a}= & N_{S, a}^{2} \\
& {\left[\left(2 E_{i} m_{N}-\frac{1}{2}\left(Q^{2}-m_{i}^{2}+m_{f}^{2}\right)\right)^{2}\left(F_{1, N}^{2}+\frac{Q^{2}}{4 m_{N}^{2}} F_{2, N}^{2}\right)\right.} \\
- & \left.\left(m_{i}^{2} Q^{2}+\frac{1}{4}\left(Q^{2}-m_{i}^{2}+m_{f}^{2}\right)^{2}\right)\left(F_{1, N}+F_{2, N}\right)^{2}\right],
\end{aligned}
$$

and for the $h_{x}^{a} \rightarrow Z^{x}$ channel:

$$
\begin{aligned}
\mathcal{I}_{H, a}= & N_{H, a}^{2}\left[-\left(m_{i}^{2} Q^{2}+\frac{1}{4}\left(Q^{2}-m_{i}^{2}+m_{f}^{2}\right)^{2}+3 m_{x}^{2} Q^{2}\right)\left(F_{1, N}+F_{2, N}\right)^{2}\right. \\
& \left.+\left\{\left(2 E_{i} m_{N}-\frac{1}{2}\left(Q^{2}-m_{i}^{2}+m_{f}^{2}\right)\right)^{2}-m_{x}^{2}\left(Q^{2}+4 m_{N}^{2}\right)\right\}\left(F_{1, N}^{2}+\frac{Q^{2}}{4 m_{N}^{2}} F_{2, N}^{2}\right)\right] .
\end{aligned}
$$

In the limit $E_{i} m_{N} \gg m_{i}^{2}, m_{f}^{2}, m_{N}^{2}$ and $Q^{2}$, all these expressions have the same approximate limit:

$$
\mathcal{I}_{\mathrm{HS}} \approx\left|N_{\mathrm{HS}}\right|^{2} 4 E_{i}^{2} m_{N}^{2} F_{1, N}^{2}\left(Q^{2}\right) .
$$

This approximation is required to derive eq. (4.23).

For the nucleon form factors, we take [22]

$$
F_{1, N}\left(Q^{2}\right) \frac{q_{N}}{\left(1+Q^{2} / m_{N}^{2}\right)^{2}}, \quad \quad F_{2, N}\left(Q^{2}\right)=\frac{\kappa_{N}}{\left(1+Q^{2} / m_{N}^{2}\right)^{2}},
$$

with $q_{p, n}=1,0$ and $\kappa_{p, n}=1.79,-1.9$. For electron scattering, we can use the same expressions with $F_{1, e}=1, F_{2, e}=0$ and consistently replacing $m_{N} \rightarrow m_{e}$.

Open Access. This article is distributed under the terms of the Creative Commons Attribution License (CC-BY 4.0), which permits any use, distribution and reproduction in any medium, provided the original author(s) and source are credited. 


\section{References}

[1] R. Essig et al., Working group report: new light weakly coupled particles, arXiv:1311.0029 [INSPIRE].

[2] J. Jaeckel and A. Ringwald, The low-energy frontier of particle physics, Ann. Rev. Nucl. Part. Sci. 60 (2010) 405 [arXiv: 1002.0329] [INSPIRE].

[3] J.L. Hewett et al., Fundamental physics at the intensity frontier, arXiv:1205.2671 [INSPIRE].

[4] B. Holdom, Two U(1)'s and epsilon charge shifts, Phys. Lett. B 166 (1986) 196 [InSPIRE].

[5] R. Foot and X.-G. He, Comment on ZZ' mixing in extended gauge theories, Phys. Lett. B 267 (1991) 509 [INSPIRE].

[6] S. Davidson, S. Hannestad and G. Raffelt, Updated bounds on millicharged particles, JHEP 05 (2000) 003 [hep-ph/0001179] [INSPIRE].

[7] P. Arias et al., WISPy cold dark matter, JCAP 06 (2012) 013 [arXiv: 1201.5902] [INSPIRE].

[8] N. Arkani-Hamed, D.P. Finkbeiner, T.R. Slatyer and N. Weiner, A theory of dark matter, Phys. Rev. D 79 (2009) 015014 [arXiv:0810.0713] [InSPIRE].

[9] N. Arkani-Hamed and N. Weiner, LHC signals for a superunified theory of dark matter, JHEP 12 (2008) 104 [arXiv:0810.0714] [INSPIRE].

[10] C. Cheung, J.T. Ruderman, L.-T. Wang and I. Yavin, Kinetic mixing as the origin of light dark scales, Phys. Rev. D 80 (2009) 035008 [arXiv:0902.3246] [InSPIRE].

[11] A. Katz and R. Sundrum, Breaking the dark force, JHEP 06 (2009) 003 [arXiv:0902.3271] [INSPIRE].

[12] D.E. Morrissey, D. Poland and K.M. Zurek, Abelian hidden sectors at a GeV, JHEP 07 (2009) 050 [arXiv:0904.2567] [INSPIRE].

[13] M. Goodsell, J. Jaeckel, J. Redondo and A. Ringwald, Naturally light hidden photons in LARGE volume string compactifications, JHEP 11 (2009) 027 [arXiv:0909.0515] [INSPIRE].

[14] R. Essig, J. Kaplan, P. Schuster and N. Toro, On the origin of light dark matter species, submitted to Phys. Rev. D (2010), arXiv:1004.0691 [INSPIRE].

[15] S. Andreas, M.D. Goodsell and A. Ringwald, Dark matter and dark forces from a supersymmetric hidden sector, Phys. Rev. D 87 (2013) 025007 [arXiv:1109.2869] [INSPIRE].

[16] M. Pospelov, Secluded U(1) below the weak scale, Phys. Rev. D 80 (2009) 095002 [arXiv:0811.1030] [INSPIRE].

[17] B. Batell, M. Pospelov and A. Ritz, Probing a secluded U(1) at B-factories, Phys. Rev. D 79 (2009) 115008 [arXiv:0903.0363] [INSPIRE].

[18] M. Reece and L.-T. Wang, Searching for the light dark gauge boson in GeV-scale experiments, JHEP 07 (2009) 051 [arXiv:0904.1743] [INSPIRE].

[19] J.D. Bjorken, R. Essig, P. Schuster and N. Toro, New fixed-target experiments to search for dark gauge forces, Phys. Rev. D 80 (2009) 075018 [arXiv:0906.0580] [INSPIRE].

[20] B. Batell, M. Pospelov and A. Ritz, Exploring portals to a hidden sector through fixed targets, Phys. Rev. D 80 (2009) 095024 [arXiv:0906.5614] [INSPIRE]. 
[21] P. deNiverville, M. Pospelov and A. Ritz, Observing a light dark matter beam with neutrino experiments, Phys. Rev. D 84 (2011) 075020 [arXiv:1107.4580] [INSPIRE].

[22] E. Izaguirre, G. Krnjaic, P. Schuster and N. Toro, New electron beam-dump experiments to search for MeV to few-GeV dark matter, Phys. Rev. D 88 (2013) 114015 [arXiv:1307.6554] [INSPIRE].

[23] M.D. Diamond and P. Schuster, Searching for light dark matter with the SLAC millicharge experiment, Phys. Rev. Lett. 111 (2013) 221803 [arXiv:1307.6861] [INSPIRE].

[24] R. Essig, J. Mardon, M. Papucci, T. Volansky and Y.-M. Zhong, Constraining light dark matter with low-energy $e^{+} e^{-}$colliders, JHEP 11 (2013) 167 [arXiv:1309.5084] [INSPIRE].

[25] P. Schuster, N. Toro and I. Yavin, Terrestrial and solar limits on long-lived particles in a dark sector, Phys. Rev. D 81 (2010) 016002 [arXiv:0910.1602] [INSPIRE].

[26] Y.F. Chan, M. Low, D.E. Morrissey and A.P. Spray, LHC signatures of a minimal supersymmetric hidden valley, JHEP 05 (2012) 155 [arXiv:1112.2705] [INSPIRE].

[27] S.P. Martin, A supersymmetry primer, hep-ph/9709356 [INSPIRE].

[28] W.-F. Chang, J.N. Ng and J.M.S. Wu, A very narrow shadow extra Z-boson at colliders, Phys. Rev. D 74 (2006) 095005 [Erratum ibid. D 79 (2009) 039902] [hep-ph/0608068] [INSPIRE].

[29] E.J. Chun, J.-C. Park and S. Scopel, Dark matter and a new gauge boson through kinetic mixing, JHEP 02 (2011) 100 [arXiv: 1011.3300] [INSPIRE].

[30] A. Hook, E. Izaguirre and J.G. Wacker, Model independent bounds on kinetic mixing, Adv. High Energy Phys. 2011 (2011) 859762 [arXiv: 1006. 0973] [INSPIRE].

[31] Particle Data Group collaboration, J. Beringer et al., Review of particle physics, Phys. Rev. D 86 (2012) 010001 [INSPIRE].

[32] H. Davoudiasl, H.-S. Lee and W.J. Marciano, Dark side of Higgs diphoton decays and muon g-2, Phys. Rev. D 86 (2012) 095009 [arXiv:1208.2973] [inSPIRE].

[33] G.F. Giudice, P. Paradisi and M. Passera, Testing new physics with the electron $g-2$, JHEP 11 (2012) 113 [arXiv:1208.6583] [InSPIRE].

[34] M. Endo, K. Hamaguchi and G. Mishima, Constraints on hidden photon models from electron $g-2$ and hydrogen spectroscopy, Phys. Rev. D 86 (2012) 095029 [arXiv:1209.2558] [INSPIRE].

[35] D. Hanneke, S. Fogwell and G. Gabrielse, New measurement of the electron magnetic moment and the fine structure constant, Phys. Rev. Lett. 100 (2008) 120801 [arXiv:0801.1134] [INSPIRE].

[36] T. Aoyama, M. Hayakawa, T. Kinoshita and M. Nio, Tenth-order QED contribution to the electron $g-2$ and an improved value of the fine structure constant, Phys. Rev. Lett. 109 (2012) 111807 [arXiv:1205.5368] [INSPIRE].

[37] R. Bouchendira, P. Clade, S. Guellati-Khelifa, F. Nez and F. Biraben, New determination of the fine structure constant and test of the quantum electrodynamics, Phys. Rev. Lett. 106 (2011) 080801 [arXiv: 1012.3627] [INSPIRE].

[38] BABAR collaboration, B. Aubert et al., Search for dimuon decays of a light scalar in radiative transitions $\tau_{3 S} \rightarrow \gamma A_{0}$, arXiv:0902.2176 [INSPIRE]. 
[39] BABAR collaboration, B. Aubert et al., Search for dimuon decays of a light scalar boson in radiative transitions $\Upsilon \rightarrow \gamma A_{0}$, Phys. Rev. Lett. 103 (2009) 081803 [arXiv:0905.4539] [INSPIRE].

[40] N. Borodatchenkova, D. Choudhury and M. Drees, Probing MeV dark matter at low-energy $e^{+} e^{-}$colliders, Phys. Rev. Lett. 96 (2006) 141802 [hep-ph/0510147] [INSPIRE].

[41] F. Archilli et al., Search for a vector gauge boson in $\phi$ meson decays with the KLOE detector, Phys. Lett. B 706 (2012) 251 [arXiv:1110.0411] [INSPIRE].

[42] KLOE-2 collaboration, D. Babusci et al., Limit on the production of a light vector gauge boson in $\phi$ meson decays with the KLOE detector, Phys. Lett. B 720 (2013) 111 [arXiv:1210.3927] [INSPIRE].

[43] WASA-AT-COSY collaboration, P. Adlarson et al., Search for a dark photon in the $\pi^{0} \rightarrow e^{+} e^{-} \gamma$ decay, Phys. Lett. B 726 (2013) 187 [arXiv:1304.0671] [INSPIRE].

[44] B. Batell, M. Pospelov and A. Ritz, Multi-lepton signatures of a hidden sector in rare B decays, Phys. Rev. D 83 (2011) 054005 [arXiv:0911.4938] [InSPIRE].

[45] J.D. Clarke, R. Foot and R.R. Volkas, Phenomenology of a very light scalar (100 MeV $<m_{h}<10 \mathrm{GeV}$ ) mixing with the SM Higgs, JHEP 02 (2014) 123 [arXiv:1310.8042] [INSPIRE].

[46] Y.G. Aditya, K.J. Healey and A.A. Petrov, Searching for super-WIMPs in leptonic heavy meson decays, Phys. Lett. B 710 (2012) 118 [arXiv:1201.1007] [INSPIRE].

[47] BABAR collaboration, B. Aubert et al., Search for invisible decays of a light scalar in radiative transitions $v_{3 S} \rightarrow \gamma A_{0}$, arXiv:0808.0017 [INSPIRE].

[48] E787 collaboration, S. Adler et al., Further search for the decay $K^{+} \rightarrow \pi^{+} \nu \bar{u} u$ in the momentum region $P<195 \mathrm{MeV} / \mathrm{c}$, Phys. Rev. D 70 (2004) 037102 [hep-ex/0403034] [INSPIRE].

[49] BNL-E949 collaboration, A.V. Artamonov et al., Study of the decay $K^{+} \rightarrow \pi^{+} \nu \bar{u} u$ in the momentum region $140<P(\pi)<199 \mathrm{MeV} / c$, Phys. Rev. D 79 (2009) 092004 [arXiv:0903.0030] [INSPIRE].

[50] BABAR collaboration, P. Harrison and H. R. Quinn, The BABAR physics book: physics at an asymmetric $B$ factory, SLAC-R-0504 (1998) [INSPIRE].

[51] R. Essig, P. Schuster and N. Toro, Probing dark forces and light hidden sectors at low-energy $e^{+} e^{-}$colliders, Phys. Rev. D 80 (2009) 015003 [arXiv:0903.3941] [InSPIRE].

[52] BABAR collaboration, B. Aubert et al., Search for a narrow resonance in $e^{+} e^{-}$to four lepton final states, arXiv:0908.2821 [INSPIRE].

[53] BaBAR collaboration, J.P. Lees et al., Search for low-mass dark-sector Higgs bosons, Phys. Rev. Lett. 108 (2012) 211801 [arXiv:1202.1313] [INSPIRE].

[54] M. Pospelov, A. Ritz and M.B. Voloshin, Secluded WIMP dark matter, Phys. Lett. B 662 (2008) 53 [arXiv:0711.4866] [INSPIRE].

[55] M. Pospelov and A. Ritz, Astrophysical signatures of secluded dark matter, Phys. Lett. B 671 (2009) 391 [arXiv:0810.1502] [INSPIRE].

[56] S. Chang, A. Pierce and N. Weiner, Momentum dependent dark matter scattering, JCAP 01 (2010) 006 [arXiv:0908.3192] [INSPIRE]. 
[57] P. Cushman et al., Working group report: WIMP dark matter direct detection, arXiv: 1310.8327 [INSPIRE].

[58] R. Essig, J. Mardon and T. Volansky, Direct detection of sub-GeV dark matter, Phys. Rev. D 85 (2012) 076007 [arXiv: 1108.5383] [InSPIRE].

[59] R. Essig, A. Manalaysay, J. Mardon, P. Sorensen and T. Volansky, First direct detection limits on sub-GeV dark matter from XENON10, Phys. Rev. Lett. 109 (2012) 021301 [arXiv:1206.2644] [INSPIRE].

[60] M. Kawasaki, K. Kohri and T. Moroi, Big-Bang nucleosynthesis and hadronic decay of long-lived massive particles, Phys. Rev. D 71 (2005) 083502 [astro-ph/0408426] [INSPIRE].

[61] K. Jedamzik, Big bang nucleosynthesis constraints on hadronically and electromagnetically decaying relic neutral particles, Phys. Rev. D 74 (2006) 103509 [hep-ph/0604251] [INSPIRE].

[62] W. Hu and J. Silk, Thermalization constraints and spectral distortions for massive unstable relic particles, Phys. Rev. Lett. 70 (1993) 2661 [inSPIRE].

[63] R. Essig, E. Kuflik, S.D. McDermott, T. Volansky and K.M. Zurek, Constraining light dark matter with diffuse X-ray and $\gamma$-ray observations, JHEP 11 (2013) 193 [arXiv:1309.4091] [INSPIRE].

[64] M. Pospelov and J. Pradler, Metastable GeV-scale particles as a solution to the cosmological lithium problem, Phys. Rev. D 82 (2010) 103514 [arXiv:1006.4172] [INSPIRE].

[65] J.B. Dent, F. Ferrer and L.M. Krauss, Constraints on light hidden sector gauge bosons from supernova cooling, arXiv:1201.2683 [INSPIRE].

[66] H.K. Dreiner, J.-F. Fortin, C. Hanhart and L. Ubaldi, Supernova constraints on MeV dark sectors from $e^{+} e^{-}$annihilations, Phys. Rev. D 89 (2014) 105015 [arXiv:1310.3826] [INSPIRE].

[67] S. Andreas, C. Niebuhr and A. Ringwald, New limits on hidden photons from past electron beam dumps, Phys. Rev. D 86 (2012) 095019 [arXiv: 1209.6083] [INSPIRE].

[68] A1 collaboration, H. Merkel et al., Search for light gauge bosons of the dark sector at the Mainz microtron, Phys. Rev. Lett. 106 (2011) 251802 [arXiv:1101.4091] [INSPIRE].

[69] APEX collaboration, S. Abrahamyan et al., Search for a new gauge boson in electron-nucleus fixed-target scattering by the APEX experiment, Phys. Rev. Lett. 107 (2011) 191804 [arXiv:1108.2750] [INSPIRE].

[70] S. Andreas, Hidden photons in beam dump experiments and in connection with dark matter, Frascati Phys. Ser. 56 (2012) 23 [arXiv:1212.4520] [INSPIRE].

[71] R. Essig, P. Schuster, N. Toro and B. Wojtsekhowski, An electron fixed target experiment to search for a new vector boson $A^{\prime}$ decaying to $e^{+} e^{-}$, JHEP 02 (2011) 009 [arXiv: 1001 .2557] [INSPIRE].

[72] M. Freytsis, G. Ovanesyan and J. Thaler, Dark force detection in low energy e-p collisions, JHEP 01 (2010) 111 [arXiv:0909.2862] [InSPIRE].

[73] Heavy Photon Search collaboration, Heavy Photon Search experiment.

[74] P.H. Adrian, The Heavy Photon Search experiment, arXiv: 1301.1103 [INSPIRE].

[75] K.J. Kim and Y.-S. Tsai, Improved Weizsacker-Williams method and its application to lepton and W boson pair production, Phys. Rev. D 8 (1973) 3109 [InSPIRE]. 
[76] T. Beranek and M. Vanderhaeghen, Study of the discovery potential for hidden photon emission at future electron scattering fixed target experiments, arXiv:1311.5104 [INSPIRE].

[77] E.M. Riordan et al., A search for short lived axions in an electron beam dump experiment, Phys. Rev. Lett. 59 (1987) 755 [INSPIRE].

[78] Y.-S. Tsai, Axion bremsstrahlung by an electron beam, Phys. Rev. D 34 (1986) 1326 [INSPIRE].

[79] J.D. Bjorken et al., Search for neutral metastable penetrating particles produced in the SLAC beam dump, Phys. Rev. D 38 (1988) 3375 [InSPIRE].

[80] A. Bross et al., A search for shortlived particles produced in an electron beam dump, Phys. Rev. Lett. 67 (1991) 2942 [INSPIRE].

[81] A. Konaka et al., Search for neutral particles in electron beam dump experiment, Phys. Rev. Lett. 57 (1986) 659 [INSPIRE].

[82] M. Davier and H. Nguyen Ngoc, An unambiguous search for a light Higgs boson, Phys. Lett. B 229 (1989) 150 [INSPIRE].

[83] S.N. Gninenko, Search for MeV dark photons in a light-shining-through-walls experiment at CERN, Phys. Rev. D 89 (2014) 075008 [arXiv:1308.6521] [INSPIRE].

[84] S. Andreas et al., Proposal for an experiment to search for light dark matter at the SPS, arXiv:1312.3309 [INSPIRE].

[85] B.C. Odom, D. Hanneke, B. D'Urso and G. Gabrielse, New measurement of the electron magnetic moment using a one-electron quantum cyclotron, Phys. Rev. Lett. 97 (2006) 030801 [Erratum ibid. 99 (2007) 039902] [INSPIRE].

[86] S.N. Gninenko, Constraints on sub-GeV hidden sector gauge bosons from a search for heavy neutrino decays, Phys. Lett. B 713 (2012) 244 [arXiv:1204.3583] [INSPIRE].

[87] P. deNiverville, D. McKeen and A. Ritz, Signatures of sub-GeV dark matter beams at neutrino experiments, Phys. Rev. D 86 (2012) 035022 [arXiv: 1205.3499] [INSPIRE].

[88] J. Blümlein and J. Brunner, New exclusion limits on dark gauge forces from proton bremsstrahlung in beam-dump data, Phys. Lett. B 731 (2014) 320 [arXiv:1311.3870] [INSPIRE].

[89] J. Blumlein and J. Brunner, New exclusion limits for dark gauge forces from beam-dump data, Phys. Lett. B 701 (2011) 155 [arXiv:1104.2747] [INSPIRE].

[90] S.N. Gninenko, Stringent limits on the $\pi^{0} \rightarrow \gamma X, X \rightarrow e^{+} e^{-}$decay from neutrino experiments and constraints on new light gauge bosons, Phys. Rev. D 85 (2012) 055027 [arXiv: 1112.5438] [INSPIRE].

[91] H.-L. Lai et al., New parton distributions for collider physics, Phys. Rev. D 82 (2010) 074024 [arXiv: 1007.2241] [INSPIRE].

[92] R. Burman and E. Smith, Parametrization of pion production and reaction cross-sections at LAMPF energies, LA-11502-MS (1989) [INSPIRE].

[93] MiniBoone collaboration, A.A. Aguilar-Arevalo et al., The neutrino flux prediction at MiniBooNE, Phys. Rev. D 79 (2009) 072002 [arXiv:0806.1449] [InSPIRE].

[94] G. Tel-Zur, Electron pair production in p-Be and p-Au collisions at $450 \mathrm{GeV} / \mathrm{c}$ (2014). 
[95] M. Bourquin and J.-M. Gaillard, Vector meson and $\psi$ contributions to single lepton spectra, Phys. Lett. B 59 (1975) 191 [INSPIRE].

[96] M. Bourquin and J.-M. Gaillard, A simple phenomenological description of hadron production, Nucl. Phys. B 114 (1976) 334 [INSPIRE].

[97] A. Alloul, N.D. Christensen, C. Degrande, C. Duhr and B. Fuks, FeynRules 2.0 - A complete toolbox for tree-level phenomenology, arXiv:1310.1921 [INSPIRE].

[98] C. Duhr and B. Fuks, A superspace module for the FeynRules package, Comput. Phys. Commun. 182 (2011) 2404 [arXiv:1102.4191] [INSPIRE].

[99] C. Degrande et al., UFO - The universal FeynRules output, Comput. Phys. Commun. 183 (2012) 1201 [arXiv: 1108. 2040] [INSPIRE].

[100] J. Alwall, M. Herquet, F. Maltoni, O. Mattelaer and T. Stelzer, MadGraph 5: going beyond, JHEP 06 (2011) 128 [arXiv:1106.0522] [INSPIRE].

[101] CHARM collaboration, F. Bergsma et al., Search for axion like particle production in $400 \mathrm{GeV}$ proton-copper interactions, Phys. Lett. B 157 (1985) 458 [INSPIRE].

[102] CHARM collaboration, F. Bergsma et al., A search for decays of heavy neutrinos in the mass range $0.5 \mathrm{GeV}$ to $2.8 \mathrm{GeV}$, Phys. Lett. B 166 (1986) 473 [INSPIRE].

[103] MINOS Collaboration, I. Ambats et al., The MINOS detectors technical design report, NUMI-L-337 (1998) [inSPIRE].

[104] MINOS collaboration, P. Adamson et al., Measurement of neutrino and antineutrino oscillations using beam and atmospheric data in MINOS, Phys. Rev. Lett. 110 (2013) 251801 [arXiv: 1304.6335] [INSPIRE].

[105] J. Blumlein et al., Limits on neutral light scalar and pseudoscalar particles in a proton beam dump experiment, Z. Phys. C 51 (1991) 341 [INSPIRE].

[106] J. Blumlein et al., Limits on the mass of light (pseudo)scalar particles from Bethe-Heitler $e^{+} e^{-}$and $\mu^{+} \mu^{-}$pair production in a proton-iron beam dump experiment, Int. J. Mod. Phys. A 7 (1992) 3835 [INSPIRE].

[107] K. Abe et al., Measurements of the T2K neutrino beam properties using the INGRID on-axis near detector, Nucl. Instrum. Meth. A 694 (2012) 211 [arXiv:1111.3119] [INSPIRE].

[108] LSND collaboration, A. Aguilar-Arevalo et al., Evidence for neutrino oscillations from the observation of anti-neutrino(electron) appearance in a anti-neutrino(muon) beam, Phys. Rev. D 64 (2001) 112007 [hep-ex/0104049] [INSPIRE].

[109] LSND collaboration, L.B. Auerbach et al., Measurement of electron-neutrino-electron elastic scattering, Phys. Rev. D 63 (2001) 112001 [hep-ex/0101039] [INSPIRE].

[110] S. Teis, W. Cassing, M. Effenberger, A. Hombach, U. Mosel et al., Pion production in heavy ion collisions at SIS energies, Z. Phys. A 356 (1997) 421 [nucl-th/9609009] [INSPIRE].

[111] V. Flaminio, W. Moorhead, D. Morrison and N. Rivoire, Compilation of cross-sections. 3. $p$ and anti-p induced reactions, CERN-HERA-73-1 (1973) [INSPIRE].

[112] T. Sjöstrand, S. Mrenna and P.Z. Skands, A brief introduction to PYTHIA 8.1, Comput. Phys. Commun. 178 (2008) 852 [arXiv:0710.3820] [INSPIRE].

[113] T. Sjöstrand, S. Mrenna and P.Z. Skands, PYTHIA 6.4 physics and manual, JHEP 05 (2006) 026 [hep-ph/0603175] [INSPIRE]. 
[114] M. Aguilar-Benitez et al., Inclusive particle production in $400 \mathrm{GeV} / \mathrm{c}$ pp interactions, Z. Phys. C 50 (1991) 405.

[115] G. Agakishiev et al., Neutral meson production in $p$ Be and $p$ Au collisions at $450 \mathrm{GeV}$ beam energy, Eur. Phys. J. C 4 (1998) 249 [INSPIRE].

[116] NA61/SHINE collaboration, N. Abgrall et al., Measurements of cross sections and charged pion spectra in proton-carbon interactions at $31 \mathrm{GeV} / \mathrm{c}$, Phys. Rev. C 84 (2011) 034604 [arXiv: 1102.0983] [INSPIRE].

[117] MINOS collaboration, P. Adamson et al., Search for sterile neutrino mixing in the MINOS long baseline experiment, Phys. Rev. D 81 (2010) 052004 [arXiv:1001.0336] [INSPIRE].

[118] French-Soviet collaboration, M. Boratav et al., Gamma production and multiplicity correlations between neutral and charged pions in pp interactions at $69 \mathrm{GeV} / \mathrm{c}$, Nucl. Phys. B 111 (1976) 529 [INSPIRE].

[119] France-Soviet Union collaboration, H. Blumenfeld et al., Photon production in $69 \mathrm{GeV}$ pp interactions, Phys. Lett. B 45 (1973) 525 [InSPIRE].

[120] T2K collaboration, K. Abe et al., The T2K experiment, Nucl. Instrum. Meth. A 659 (2011) 106 [arXiv: 1106.1238] [inSPIRE].

[121] NOMAD collaboration, P. Astier et al., Search for heavy neutrinos mixing with $\tau$ neutrinos, Phys. Lett. B 506 (2001) 27 [hep-ex/0101041] [INSPIRE].

[122] G. Bernardi et al., Search for neutrino decay, Phys. Lett. B 166 (1986) 479 [InSPIRE].

[123] G. Bernardi et al., Further limits on heavy neutrino couplings, Phys. Lett. B 203 (1988) 332 [INSPIRE].

[124] MiniBooNE collaboration, A.A. Aguilar-Arevalo et al., The MiniBooNE detector, Nucl. Instrum. Meth. A 599 (2009) 28 [arXiv:0806.4201] [INSPIRE].

[125] S. Assylbekov et al., The T2K ND280 off-axis Pi-Zero detector, Nucl. Instrum. Meth. A 686 (2012) 48 [arXiv:1111.5030] [InSPIRE].

[126] A.S. Kronfeld et al., Project X: physics opportunities, arXiv:1306.5009 [INSPIRE].

[127] S. Holmes, S. Nagaitsev and R. Tschirhart, Project X: a flexible high power proton facility, arXiv:1305.3809 [INSPIRE].

[128] S.J. Brodsky, F. Fleuret, C. Hadjidakis and J.P. Lansberg, Physics opportunities of a fixed-target experiment using the LHC beams, Phys. Rept. 522 (2013) 239 [arXiv:1202.6585] [INSPIRE].

[129] J.P. Lansberg et al., A Fixed-Target ExpeRiment at the LHC (AFTER@LHC): luminosities, target polarisation and a selection of physics studies, PoS (QNP2012) 049 [arXiv: 1207.3507] [INSPIRE].

[130] M.J. Strassler, Possible effects of a hidden valley on supersymmetric phenomenology, hep-ph/0607160 [INSPIRE].

[131] M. Baumgart, C. Cheung, J.T. Ruderman, L.-T. Wang and I. Yavin, Non-abelian dark sectors and their collider signatures, JHEP 04 (2009) 014 [arXiv:0901.0283] [INSPIRE].

[132] C. Cheung, J.T. Ruderman, L.-T. Wang and I. Yavin, Lepton jets in (supersymmetric) electroweak processes, JHEP 04 (2010) 116 [arXiv:0909.0290] [INSPIRE]. 
[133] T. Beranek, H. Merkel and M. Vanderhaeghen, Theoretical framework to analyze searches for hidden light gauge bosons in electron scattering fixed target experiments, Phys. Rev. D 88 (2013) 015032 [arXiv:1303.2540] [INSPIRE].

[134] J.R. Andersen, M. Rauch and M. Spannowsky, Dark sector spectroscopy at the ILC, arXiv: 1308.4588 [INSPIRE].

[135] A. Kumar, D.E. Morrissey and A. Spray, Kinetically-enhanced anomaly mediation, JHEP 12 (2011) 013 [arXiv: 1109.1565] [INSPIRE].

[136] J.F. Donoghue, J. Gasser and H. Leutwyler, The decay of a light Higgs boson, Nucl. Phys. B 343 (1990) 341 [INSPIRE].

[137] F. Bezrukov and D. Gorbunov, Light inflaton hunter's guide, JHEP 05 (2010) 010 [arXiv:0912.0390] [INSPIRE].

[138] F. Bezrukov and D. Gorbunov, Light inflaton after LHC8 and WMAP9 results, JHEP 07 (2013) 140 [arXiv: 1303.4395] [inSPIRE].

[139] Y.-S. Tsai, Pair production and bremsstrahlung of charged leptons, Rev. Mod. Phys. 46 (1974) 815 [Erratum ibid. 49 (1977) 521-423] [INSPIRE]. 